\title{
Total Synthesis of Sandresolide B and Amphilectolide
}

\author{
Ingrid T. Chen, Irina Baitinger, Lucas Schreyer, and Dirk Trauner* \\ Department of Chemistry and Center of Integrated Protein Science, University of \\ Munich, Butenandtstraße 5 - 13 (F4.086), 81377 Munich, Germany
}

\section{Supporting Information}

General Experimental Details: All reactions were carried out under an inert $\mathrm{N}_{2}$ atmosphere in oven-dried glassware. Flash column chromatography was carried out with Merck 40-60 $\mu \mathrm{M} 60 \AA$ silica gel. Reactions and chromatography fractions were monitored with Merck silica gel $60 \mathrm{~F}_{254}$ plates and visualized with potassium permanganate, ceric ammonium molybdate, or anisaldehyde. Tetrahydrofuran (THF), and diethyl ether $\left(\mathrm{Et}_{2} \mathrm{O}\right)$ were distilled from sodium benzophenone ketyl. $n$-Butyllithium $(n \mathrm{BuLi})$ was titrated with diphenylacetic acid prior to use. All other reagents and solvents were used without further purification from commercial sources. Organic extracts were dried over $\mathrm{MgSO}_{4}$ unless otherwise noted.

Instrumentation: FT-IR spectra were obtained as neat samples on a Perkin-Elmer BXIIFTIR spectrometer. Proton and carbon NMR spectra ( ${ }^{1} \mathrm{H}$ NMR and ${ }^{13} \mathrm{C}$ NMR) were recorded in deuterated chloroform $\left(\mathrm{CDCl}_{3}\right)$ (unless otherwise noted) on a Varian Mercury $400 \mathrm{MHz}$ or $600 \mathrm{MHz}$ or Bruker Avance III HD $400 \mathrm{MHz}$ spectrometer, reported in ppm and calibrated to residual solvent peaks. Multiplicities are abbreviated as follows: $\mathrm{s}=$ singlet, $\mathrm{d}=$ doublet, $\mathrm{t}=$ triplet, $\mathrm{q}=$ quartet, $\mathrm{br}=$ broad, $\mathrm{m}=$ multiplet. Optical rotations were measured on a Perkin-Elmer 241 Polarimeter. High resolution mass spectra (HRMS) were obtained at Ludwig-Maximilians-Universität using electron impact (EI) or electrospray ionization (ESI).

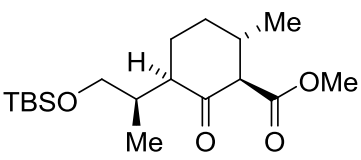

$(1 R, 3 R, 6 S)$-methyl 3-((R)-1-((tert-butyldimethylsilyl)oxy)propan-2-yl)-6-methyl-2oxocyclohexanecarboxylate (11). To a solution of $12.0 \mathrm{~mL}(8.63 \mathrm{~g}, 85.3 \mathrm{mmol})$ diisopropylamine in $400 \mathrm{~mL}$ THF cooled to $-78{ }^{\circ} \mathrm{C}$ was added $51.6 \mathrm{~mL}(82.5 \mathrm{mmol})$ of $n \mathrm{BuLi}$ (1.6 $\mathrm{M}$ in hexanes) dropwise. The solution was allowed to come to $0{ }^{\circ} \mathrm{C}$ for 2 hours, at which point it was cooled back to $-78{ }^{\circ} \mathrm{C}$. A solution of $7.82 \mathrm{~g}(27.5 \mathrm{mmol}) \mathbf{1 0}$ in $100 \mathrm{~mL}$ THF was then added dropwise and the reaction mixture was stirred for another hour before addition of $14.8 \mathrm{~mL}(15.3 \mathrm{~g}, 85.2 \mathrm{mmol})$ hexamethylphosphoramide (HMPA) followed by $6.76 \mathrm{~mL}(7.25 \mathrm{~g}, 85.3 \mathrm{mmol})$ methyl cyanoformate (Mander's reagent). The solution became pale yellow and after 15 minutes at $-78{ }^{\circ} \mathrm{C}$, was quenched with $\mathrm{H}_{2} \mathrm{O}$. The layers were separated and the aqueous layer was extracted with $\mathrm{Et}_{2} \mathrm{O}$. The combined organic layers were washed with $\mathrm{NaCl}$ (saturated), dried over $\mathrm{Na}_{2} \mathrm{SO}_{4}$, filtered, 
and evaporated. Purification by flash column chromatography (5 to $15 \% \mathrm{Et}_{2} \mathrm{O} / \mathrm{hexanes}$ ) afforded $7.81 \mathrm{~g}$ of $\mathbf{1 1}(83 \%)$ as a pale yellow oil.

Rf: 0.41, 10\% EtOAc/hexanes. ${ }^{1} \mathrm{H}$ NMR (400 MHz): $\delta 3.76(\mathrm{~s}, 3 \mathrm{H}), 3.49(\mathrm{dd}, 1 \mathrm{H}, J=$ 9.9, $5.5 \mathrm{~Hz}), 3.37(\mathrm{dd}, 1 \mathrm{H}, J=9.9,7.8 \mathrm{~Hz}), 3.04(\mathrm{dd}, 1 \mathrm{H}, J=12.2,1.2 \mathrm{~Hz}), 2.53(\mathrm{~m}, 1 \mathrm{H})$, $2.25(\mathrm{~m}, 2 \mathrm{H}), 1.99(\mathrm{~m}, 2 \mathrm{H}), 1.45(\mathrm{~m}, 2 \mathrm{H}), 1.02(\mathrm{~d}, 3 \mathrm{H}, J=6.4 \mathrm{~Hz}), 0.87(\mathrm{~s}, 9 \mathrm{H}), 0.80(\mathrm{~d}$, $3 \mathrm{H}, J=7.0 \mathrm{~Hz}), 0.02(\mathrm{~s}, 3 \mathrm{H}), 0.01(\mathrm{~s}, 3 \mathrm{H}) .{ }^{13} \mathrm{C} \mathrm{NMR}(100 \mathrm{MHz}): \delta 207.05,170.53$, $65.77,65.70,52.01,49.97,37.56,33.34,33.05,26.46,26.07,21.24,18.42,12.77,-5.23$, -5.33. IR: 2955, 2930, 2857, 1748, 1472, 1359, 1251, $1090 \mathrm{~cm}^{-1}$. HRMS (ESI) calcd for $\mathrm{C}_{18} \mathrm{H}_{34} \mathrm{O}_{4} \mathrm{SiNa}\left([\mathrm{M}+\mathrm{Na}]^{+}\right)$365.2124, found 365.2117. $[\alpha]^{25}{ }_{\mathrm{D}}+8.4\left(c=1.0, \mathrm{CHCl}_{3}\right)$.

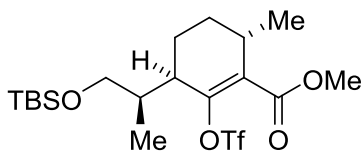

(3R,6S)-methyl 3-((R)-1-((tert-butyldimethylsilyl)oxy)propan-2-yl)-6-methyl-2(((trifluoromethyl)sulfonyl)oxy)cyclohex-1-enecarboxylate (S1). To a solution of 0.45 $\mathrm{mL}(3.24 \mathrm{mmol}) \mathrm{DIA}$ in $50 \mathrm{~mL}$ THF at $0^{\circ} \mathrm{C}$ was added $1.30 \mathrm{~mL}(3.24 \mathrm{mmol}) \mathrm{nBuLi}(2.5$ $\mathrm{M}$ in hexanes). The yellow solution was stirred for 20 minutes, then cooled to $-78{ }^{\circ} \mathrm{C}$ and a solution of $0.701 \mathrm{~g}(2.16 \mathrm{mmol}) 11$ in $10 \mathrm{~mL}$ THF was added dropwise. After an hour, $0.55 \mathrm{~mL}(3.24 \mathrm{mmol}) \mathrm{Tf}_{2} \mathrm{O}$ was added dropwise. The solution became a darker yellow color and after 15 minutes, complete consumption of starting material was observed by TLC. The reaction mixture was then quenched with $\mathrm{NaHCO}_{3}$ (saturated). The layers were separated and the organic layer was dried over $\mathrm{MgSO}_{4}$, filtered, and evaporated then purified by flash column chromatography (5 to 10\% EtOAc/hexanes, 1\% TEA) to afford $833 \mathrm{mg}$ of $\mathbf{S 1}(86 \%)$ as a pale yellow oil, (92\% based on recovered starting material (borsm), reverse reaction may have occurred on column).

Rf: $0.59,10 \%$ EtOAc/hexanes. ${ }^{1} \mathrm{H}$ NMR (400 MHz): $\delta 3.81(\mathrm{~s}, 3 \mathrm{H}), 3.53(\mathrm{dd}, 1 \mathrm{H}, J=$ 10.0, $6.0 \mathrm{~Hz}), 3.41(\mathrm{~m}, 1 \mathrm{H}), 2.86(\mathrm{~m}, 1 \mathrm{H}), 2.75(\mathrm{~m}, 1 \mathrm{H}), 2.20(\mathrm{~m}, 1 \mathrm{H}), 1.81(\mathrm{~m}, 2 \mathrm{H}), 1.53$ $(\mathrm{m}, 1 \mathrm{H}), 1.31(\mathrm{~m}, 1 \mathrm{H}), 1.11(\mathrm{~d}, 3 \mathrm{H}, J=6.9 \mathrm{~Hz}), 0.88(\mathrm{~s}, 9 \mathrm{H}), 0.76(\mathrm{~d}, 3 \mathrm{H}, J=7.0 \mathrm{~Hz})$, 0.03 (s, 6H). ${ }^{13} \mathrm{C}$ NMR (100 MHz): $\delta 165.49,152.14,131.16,118.46$ (q, $J=320.2 \mathrm{~Hz}$, $\left.\mathrm{CF}_{3}\right), 65.19,52.12,37.94,35.49,32.16,28.64,25.93,20.32,20.14,18.34,11.46,-5.38$, -5.55. IR: 2933, 2859, 1733, 1472, 1422, 1246, 1140, $1068 \mathrm{~cm}^{-1}$. HRMS (ESI) calcd for $\mathrm{C}_{19} \mathrm{H}_{33} \mathrm{O}_{6} \mathrm{~F}_{3} \mathrm{SSiNa}\left([\mathrm{M}+\mathrm{Na}]^{+}\right) 497.1617$, found 497.1611. $[\alpha]^{25}{ }_{\mathrm{D}}-5.3\left(c=0.87, \mathrm{CHCl}_{3}\right)$.

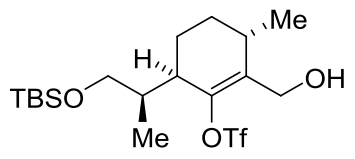

(3S,6R)-6-((R)-1-((tert-butyldimethylsilyl)oxy)propan-2-yl)-2-(hydroxymethyl)-3methylcyclohex-1-en-1-yl trifluoromethanesulfonate (12). A solution of $10.1 \mathrm{~g}(21.3$ 
mmol) $\mathbf{S 1}$ in $600 \mathrm{~mL}$ DCM was cooled to to $-78{ }^{\circ} \mathrm{C}$, and then $60.2 \mathrm{~mL}$ of DIBAL-H (6.02 mmol, $1.0 \mathrm{M}$ in $\mathrm{PhCH}_{3}$ ) was added dropwise. After 1 hour of stirring, the reaction mixture was warmed to room temperature for 10 minutes, at which point it was quenched with a 1:1 mixture of $\mathrm{H}_{2} \mathrm{O} /$ Rochelle's Salt (saturated) and diluted with DCM. The layers were stirred vigorously until no emulsion was present. The layers were then separated and the aqueous layer was extracted twice with DCM. The combined organic layers were dried over $\mathrm{Na}_{2} \mathrm{SO}_{4}$, filtered, and evaporated. Purification by flash column chromatography (10 to $20 \%$ EtOAc/hexanes and 1\% TEA) afforded $7.90 \mathrm{~g}$ of $\mathbf{1 2}(83 \%)$ as a clear, colorless oil.

Rf: 0.55, 25\% EtOAc/hexanes. ${ }^{1} \mathrm{H}$ NMR $(400 \mathrm{MHz}): \delta 4.36(\mathrm{~d}, 1 \mathrm{H}, J=12.7 \mathrm{~Hz}), 4.24$ (dd, $1 \mathrm{H}, J=12.7,1.9 \mathrm{~Hz}), 3.50(\mathrm{dd}, 1 \mathrm{H}, J=10.0,6.2 \mathrm{~Hz}), 3.42(\mathrm{dd}, 1 \mathrm{H}, J=10.0,8.8$ $\mathrm{Hz}), 2.85(\mathrm{~m}, 1 \mathrm{H}), 2.55(\mathrm{~m}, 1 \mathrm{H}), 2.17(\mathrm{~m}, 1 \mathrm{H}), 1.80(\mathrm{br} \mathrm{m}, 2 \mathrm{H}), 1.69$ (br s, $1 \mathrm{H}, \mathrm{OH}), 1.45$ $(\mathrm{m}, 1 \mathrm{H}), 1.29(\mathrm{~m}, 1 \mathrm{H}), 1.19(\mathrm{~d}, 3 \mathrm{H}, J=7.0 \mathrm{~Hz}), 0.88(\mathrm{~s}, 9 \mathrm{H}), 0.74(\mathrm{~d}, 3 \mathrm{H}, J=7.0 \mathrm{~Hz})$, $0.03(\mathrm{~m}, 6 \mathrm{H}) .{ }^{13} \mathrm{C}$ NMR $(100 \mathrm{MHz}): \delta 147.37,136.36,118.53\left(\mathrm{q}, J=319.9 \mathrm{~Hz}, \mathrm{CF}_{3}\right)$, $65.38,58.41,38.45,35.20,32.35,29.91,25.93,21.17,19.58,18.33,11.22,-5.35,-5.50$. IR: $3342,2931,1673,1473,1415,1248,1140,1090,972,883,819,775,667 \mathrm{~cm}^{-1}$. HRMS (ESI) calcd for $\mathrm{C}_{18} \mathrm{H}_{33} \mathrm{O}_{5} \mathrm{ClF}_{3} \mathrm{SiS}\left([\mathrm{M}+\mathrm{Cl}]^{-}\right) 481.1459$, found $481.1458 .[\alpha]^{25}{ }_{\mathrm{D}}^{-}$ $12\left(c=0.33, \mathrm{CHCl}_{3}\right)$.

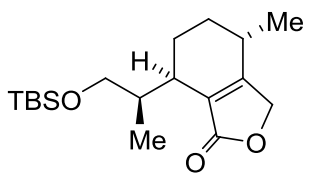

(4S,7R)-7-((R)-1-((tert-butyldimethylsilyl)oxy)propan-2-yl)-4-methyl-4,5,6,7tetrahydroisobenzofuran-1(3H)-one (13). We present two procedures to provide this compound in $98 \%$ yield.

1) To a solution of $7.90 \mathrm{~g}(17.7 \mathrm{mmol}) \mathbf{1 2}$ in $90 \mathrm{~mL} \mathrm{MeCN}$ was added $2.45 \mathrm{~g}(2.12$ mmol) $\mathrm{Pd}\left(\mathrm{PPh}_{3}\right)_{4}, 0.825 \mathrm{~g}(19.5 \mathrm{mmol}) \mathrm{LiCl}$ (dried under high vacuum), and $8.43 \mathrm{~mL}$ $(6.56 \mathrm{~g}, 35.4 \mathrm{mmol}) \mathrm{NBu}_{3}$. The solution was degassed with $\mathrm{N}_{2}$ for 20 minutes, then transferred to a Paar bomb. The apparatus was filled with $\mathrm{CO}(3 \mathrm{bar})$ and flushed three times, then filled with 3 bar $\mathrm{CO}$ and heated to $85^{\circ} \mathrm{C}$. The solution became orange after 5 minutes. After 48 hours, the solution was cooled to room temperature and the apparatus was flushed with $\mathrm{N}_{2}$ before it was opened. The solvent was evaporated, and flash column chromatography (10\% EtOAc/hexanes) afforded $5.6 \mathrm{~g}(98 \%)$ of $\mathbf{1 3}$ as a light yellow oil.

2) To a solution of $2.67 \mathrm{~g}(5.98 \mathrm{mmol}) \mathbf{1 2}$ in $\mathrm{MeCN}(95 \mathrm{~mL}$, previously degassed by 3 freeze-pump-thaw cycles) in a $300 \mathrm{~mL}$ Schlenk tube was subsequently added $2.80 \mathrm{~mL}$ (2.22 g, $11.8 \mathrm{mmol}) \mathrm{NBu}_{3}, 829 \mathrm{mg}(0.72 \mathrm{mmol}) \mathrm{Pd}\left(\mathrm{PPh}_{3}\right)_{4}$ and $279 \mathrm{mg}(6.58 \mathrm{mmol}) \mathrm{LiCl}$ (previously dried in vacuo at $200{ }^{\circ} \mathrm{C}$ overnight). $1.00 \mathrm{~mL}(1.84 \mathrm{~g}, 96 \% \mathrm{w} / \mathrm{w}, 18.0 \mathrm{mmol})$ $\mathrm{H}_{2} \mathrm{SO}_{4}$ was placed in a $50 \mathrm{~mL}$ Schlenk tube. Both Schlenk tubes were connected with each other by a plastic tube $(1.5 \mathrm{~cm}$ outer diameter, $0.2 \mathrm{~cm}$ thickness $)$. The septa on both Schlenk tubes were sealed with Teflon ${ }^{\circledR}$ tape, Parafilm ${ }^{\circledR}$ and secured with springs (see 
Figure 1). A blast shield was placed in front of the reaction setup as a precautionary measure. Both Schlenk tubes were then heated to $75{ }^{\circ} \mathrm{C}$, at which point $0.71 \mathrm{~mL}(0.87 \mathrm{~g}, 95 \% \mathrm{w} / \mathrm{w}$, $17.9 \mathrm{mmol}) \mathrm{HCO}_{2} \mathrm{H}$ was added to the stirred $\mathrm{H}_{2} \mathrm{SO}_{4}$ via syringe. After 23 hours, both Schlenk tubes were cooled to room temperature and the reaction mixture was filtered through a silica pad, which was washed with EtOAc. The solvents were removed in vacuo, and the crude product was purified by flash column chromatography ( 9 to 13 to $17 \% \mathrm{EtOAc} / n$-pentane), yielding $1.90 \mathrm{~g}(98 \%)$ of $\mathbf{1 3}$ as a colorless oil.

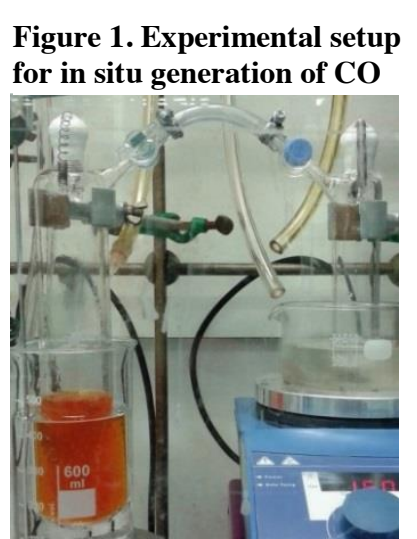

Rf: $0.55,25 \%$ EtOAc/hexanes. ${ }^{1} \mathrm{H}$ NMR (400 MHz): $\delta 4.73$ (ddd, $1 \mathrm{H}, J=17.1,3.3,1.0$ Hz) 4.66 (ddd, $1 \mathrm{H}, J=17.1,2.6,1.5 \mathrm{~Hz}$ ), 3.52 (dd, $1 \mathrm{H}, J=10.0,6.5 \mathrm{~Hz}), 3.48$ (dd, $1 \mathrm{H}, J$ $=10.0,8.3 \mathrm{~Hz}), 2.79(\mathrm{br} \mathrm{m}, 2 \mathrm{H}), 2.51(\mathrm{~m}, 1 \mathrm{H}), 1.98(\mathrm{~m}, 1 \mathrm{H}), 1.78(\mathrm{~m}, 1 \mathrm{H}), 1.43(\mathrm{~m}, 1 \mathrm{H})$, $1.28(\mathrm{~m}, 1 \mathrm{H}), 1.11(\mathrm{~d}, 3 \mathrm{H}, J=7.1 \mathrm{~Hz}), 0.88(\mathrm{~s}, 9 \mathrm{H}), 0.65(\mathrm{~d}, 3 \mathrm{H}, J=6.9 \mathrm{~Hz}), 0.05(\mathrm{~s}$, $3 \mathrm{H}), 0.04(\mathrm{~s}, 3 \mathrm{H}) .{ }^{13} \mathrm{C}$ NMR $(100 \mathrm{MHz}): \delta 173.72,166.28,127.73,70.02,65.98,34.13$, $33.43,30.88,30.47,26.07,21.37,18.77,18.40,11.51,-5.17,-5.32$. IR: 2929, 1750, $1661,1462,1254,1086,1020 \mathrm{~cm}^{-1}$. HRMS (ESI) calcd for $\mathrm{C}_{18} \mathrm{H}_{33} \mathrm{O}_{3} \mathrm{Si}\left([\mathrm{M}+\mathrm{H}]^{+}\right)$ 325.2199, found 325.2194. $[\alpha]^{25}{ }_{\mathrm{D}}+43\left(c=0.33, \mathrm{CHCl}_{3}\right)$.

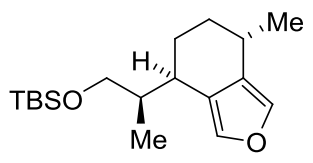

tert-butyldimethyl $((R)-2-((4 R, 7 S)$-7-methyl-4,5,6,7-tetrahydroisobenzofuran-4yl)propoxy)silane (14). A solution of $5.61 \mathrm{~g}(17.3 \mathrm{mmol}) \mathbf{1 3}$ in $180 \mathrm{~mL} \mathrm{PhCH}_{3}$ was cooled to to $-78{ }^{\circ} \mathrm{C}$, and then $27.7 \mathrm{~mL}$ of a DIBAL-H solution in $\mathrm{PhCH}_{3}(1.0 \mathrm{M}, 27.7$ mmol) was added dropwise, the solution was left to stir for $30 \mathrm{~min}$. After warming the reaction mixture to $0{ }^{\circ} \mathrm{C}$, it was quenched with a 1:1 mixture of $\mathrm{H}_{2} \mathrm{O}$ /Rochelle's Salt (saturated) and diluted with DCM. The layers were stirred vigorously until no emulsion was present. The layers were then separated and the aqueous layer was extracted twice with DCM. The combied organic layers were washed with $\mathrm{NaCl}$ (saturated), dried over $\mathrm{Na}_{2} \mathrm{SO}_{4}$, filtered, and evaporated. To the crude mixture were added $35.5 \mathrm{~g}$ silica gel and $\sim 300 \mathrm{~mL} \mathrm{CHCl}$, then stirred for 16 hours. This mixture was then filtered, washed with $\mathrm{Et}_{2} \mathrm{O}$, and evaporated. Purification by flash column chromatography $(5 \%$ EtOAc/hexanes) afforded $4.67 \mathrm{~g}$ of $\mathbf{1 4}(88 \%)$ as a clear, colorless oil.

Rf: $0.61,10 \%$ EtOAc/hexanes. ${ }^{1} \mathrm{H}$ NMR (600 MHz): $\delta 7.18$ (s, 1H), $7.14(\mathrm{~s}, 1 \mathrm{H}), 3.55$ $(\mathrm{m}, 2 \mathrm{H}), 2.90(\mathrm{~m}, 1 \mathrm{H}), 2.58(\mathrm{~m}, 1 \mathrm{H}), 2.07(\mathrm{~m}, 1 \mathrm{H}), 1.88(\mathrm{~m}, 1 \mathrm{H}), 1.71(\mathrm{~m}, 1 \mathrm{H}), 1.28(\mathrm{~m}$, $1 \mathrm{H}), 1.21(\mathrm{~d}, 3 \mathrm{H}, J=7 \mathrm{~Hz}$ and $\mathrm{m}, 1 \mathrm{H}), 0.90(\mathrm{~s}, 9 \mathrm{H}), 0.79(\mathrm{~d}, 3 \mathrm{H}, J=7 \mathrm{~Hz}), 0.06(\mathrm{~d}, 6 \mathrm{H}, J$ $=2 \mathrm{~Hz}) .{ }^{13} \mathrm{C}$ NMR $(100 \mathrm{MHz}): \delta 137.26,137.11,128.78,125.04,66.17,39.12,33.42$, $32.70,27.86,25.93,23.34,21.13,18.30,11.87,-5.32$, -5.38. IR: 2956, 2856, 1462, $1415,1361,1250,1208,1141,1089 \mathrm{~cm}^{-1}$. HRMS (EI) calcd for $\mathrm{C}_{18} \mathrm{H}_{32} \mathrm{O}_{2} \mathrm{Si}\left([\mathrm{M}]^{+}\right)$ 308.2172, found 308.2172. $[\alpha]^{25}{ }_{\mathrm{D}}+56\left(c=0.33, \mathrm{CHCl}_{3}\right)$. 


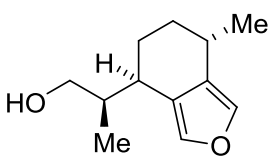

(R)-2-((4R,7S)-7-methyl-4,5,6,7-tetrahydroisobenzofuran-4-yl)propan-1-ol (7). A solution of $4.60 \mathrm{~g}(14.9 \mathrm{mmol}) 14 \mathrm{in} 150 \mathrm{~mL}$ THF was cooled to $0{ }^{\circ} \mathrm{C}$, and then $20.9 \mathrm{~mL}$ (20.9 mmol) of a tetrabutylammonium fluoride (TBAF) solution (1.0 M in THF) was added dropwise. After 1 hour, the solution was then warmed to room temperature and stirred for another 30 minutes. The reaction mixture was then quenched with $\mathrm{NaHCO}_{3}$ (saturated), diluted with $\mathrm{Et}_{2} \mathrm{O}$, and the layers were separated. The aqueous layer was extracted twice with $\mathrm{Et}_{2} \mathrm{O}$ and the combined organic layers was washed with $\mathrm{NaCl}$ (saturated), dried over $\mathrm{Na}_{2} \mathrm{SO}_{4}$, filtered, and evaporated. Flash column chromatography (25\% EtOAc/hexanes) afforded $2.90 \mathrm{~g}$ (quantitative yield) of 7 as a clear, colorless oil.

Rf: 0.35, 25\% EtOAc/hexanes. ${ }^{1} \mathrm{H}$ NMR (400 MHz): $\delta 7.19(\mathrm{~s}, 1 \mathrm{H}), 7.17(\mathrm{~s}, 1 \mathrm{H}), 3.63$ $(\mathrm{m}, 2 \mathrm{H}), 2.89(\mathrm{~m}, 1 \mathrm{H}), 2.59(\mathrm{~m}, 1 \mathrm{H}), 2.11(\mathrm{~m}, 1 \mathrm{H}), 1.90(\mathrm{~m}, 1 \mathrm{H}), 1.76(\mathrm{~m}, 1 \mathrm{H}), 1.51(\mathrm{br}$ $\mathrm{s}, 1 \mathrm{H}), 1.34(\mathrm{~m}, 2 \mathrm{H}), 1.22(\mathrm{~d}, 3 \mathrm{H}, J=9 \mathrm{~Hz}), 0.88(\mathrm{~d}, 3 \mathrm{H}, J=9 \mathrm{~Hz}) .{ }^{13} \mathrm{C} \mathrm{NMR}(75 \mathrm{MHz})$ : $\delta 137.34,137.15,128.75,124.62,66.27,39.35,33.76,32.63,27.83,23.67,21.11,12.12$. IR: 3333, 2956, 1538, 1453, 1374, 1232, 1129, 1024, $890 \mathrm{~cm}^{-1}$. HRMS (EI) calcd for $\mathrm{C}_{12} \mathrm{H}_{18} \mathrm{O}_{2}\left([\mathrm{M}]^{+}\right)$194.1307, found 194.1303. $[\alpha]^{25}{ }_{\mathrm{D}}+76\left(c=0.40, \mathrm{CHCl}_{3}\right)$.

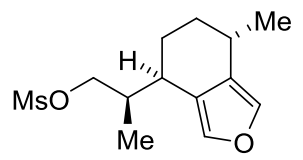

(R)-2-((4R,7S)-7-methyl-4,5,6,7-tetrahydroisobenzofuran-4-yl)propyl

methanesulfonate (21). To a solution of $3.05 \mathrm{~g}(15.7 \mathrm{mmol}) 7$ in $300 \mathrm{~mL} \mathrm{DCM}$ was added $3.27 \mathrm{~mL}(2.38 \mathrm{~g}, 23.5 \mathrm{mmol}) \mathrm{NEt}_{3}$ and $1.46 \mathrm{~mL}(2.16 \mathrm{~g}, 18.8 \mathrm{mmol})$ mesyl chloride. The solution was stirred at room temperature for 30 minutes, and quenched with $\mathrm{NaHCO}_{3}$ (saturated), extracted with $\mathrm{CHCl}_{3}$, dried over $\mathrm{Na}_{2} \mathrm{SO}_{4}$, filtered, and evaporated. Purification by flash column chromatography (25\% EtOAc/hexanes) afforded $4.14 \mathrm{~g}(97 \%)$ of $\mathbf{2 1}$ as a slightly tan oil.

Rf: 0.66, 40\% EtOAc/hexanes. ${ }^{1} \mathrm{H}$ NMR (400 MHz): $\delta 7.19(\mathrm{~d}, 1 \mathrm{H}, J=1.6 \mathrm{~Hz}), 7.17(\mathrm{~d}$, $1 \mathrm{H}, J=1.6 \mathrm{~Hz}), 4.23(\mathrm{dd}, 1 \mathrm{H}, J=9.7 \mathrm{~Hz}), 4.15(\mathrm{dd}, 1 \mathrm{H}, J=9.7 \mathrm{~Hz}), 3.02(\mathrm{~s}, 3 \mathrm{H}), 2.89$ $(\mathrm{m}, 1 \mathrm{H}), 2.57(\mathrm{~m}, 1 \mathrm{H}), 2.35(\mathrm{~m}, 1 \mathrm{H}), 1.92(\mathrm{~m}, 1 \mathrm{H}), 1.77(\mathrm{~m}, 1 \mathrm{H}), 1.33$ (ddd, 1H, J = 14 $\mathrm{Hz}), 1.21(\mathrm{~m}, 4 \mathrm{H}), 0.94(\mathrm{~d}, 3 \mathrm{H}, J=7 \mathrm{~Hz}) .{ }^{13} \mathrm{C}$ NMR $(75 \mathrm{MHz}): \delta 137.61,137.18$, 128.57, 123.46, 72.42, 37.34, 36.50, 33.68, 32.35, 27.70, 23.79, 21.05, 12.14. IR: 2959, $2361,1540,1455,1353,1129,1042 \mathrm{~cm}^{-1}$. HRMS (ESI) calcd for $\mathrm{C}_{13} \mathrm{H}_{20} \mathrm{O}_{4} \mathrm{SCl}([\mathrm{M}+$ $\left.\mathrm{Cl}]^{-}\right)$307.0771, found 307.0775. $[\alpha]_{\mathrm{D}}^{25}+49\left(c=0.47, \mathrm{CHCl}_{3}\right)$. 


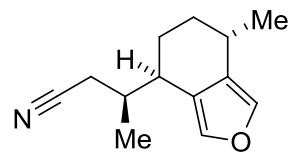

(S)-3-((4R,7S)-7-methyl-4,5,6,7-tetrahydroisobenzofuran-4-yl)butanenitrile (15). To a solution of $130 \mathrm{mg}(0.477 \mathrm{mmol})$ of 21 in $12 \mathrm{~mL} \mathrm{DMF}$ (anhydrous) in a pressure tube was added $68 \mathrm{mg}(1.049 \mathrm{mmol})$ of $\mathrm{KCN}$. The mixture was heated to $70{ }^{\circ} \mathrm{C}$ for 2.5 hours, then to $95{ }^{\circ} \mathrm{C}$ for 4 hours. The reaction mixture was then cooled to $70{ }^{\circ} \mathrm{C}$ for 16 hours, quenched with $\mathrm{NaHCO}_{3}$ (saturated), and diluted with $\mathrm{Et}_{2} \mathrm{O}$. The layers were separated and the organic layer was dried over $\mathrm{MgSO}_{4}$, filtered, and evaporated. Flash column chromatography (15\% EtOAc/hexanes) afforded $86 \mathrm{mg}(89 \%)$ of 15 as a white solid.

Rf: 0.61, 25\% EtOAc/hexanes. ${ }^{1} \mathrm{H}$ NMR (600 MHz): $\delta 7.20(\mathrm{~d}, 1 \mathrm{H}, J=1.6 \mathrm{~Hz}), 7.17(\mathrm{~d}$, $1 \mathrm{H}, J=1.5 \mathrm{~Hz}), 2.86(\mathrm{~m}, 1 \mathrm{H}), 2.58(\mathrm{~m}, 1 \mathrm{H}), 2.34(\mathrm{~m}, 2 \mathrm{H}), 2.31(\mathrm{~m}, 1 \mathrm{H}), 1.91(\mathrm{~m}, 1 \mathrm{H})$, $1.78(\mathrm{~m}, 1 \mathrm{H}), 1.35(\mathrm{ddd}, 1 \mathrm{H}, J=11 \mathrm{~Hz}), 1.21(\mathrm{~m}, 1 \mathrm{H}$ and d, 3H, $J=6.7 \mathrm{~Hz}), 1.05(\mathrm{~d}, 3 \mathrm{H}$, $J=6.8 \mathrm{~Hz}) .{ }^{13} \mathrm{C}$ NMR $(150 \mathrm{MHz}): \delta 137.67,137.30,128.42,123.11,119.22,36.49$, 34.31, 32.09, 27.61, 23.91, 22.15, 21.02, 15.50. IR: 3104, 2942, 2243, 1541, 1457, 1328, 1266, 1122, $1035 \mathrm{~cm}^{-1}$. HRMS (EI) calcd for $\mathrm{C}_{13} \mathrm{H}_{17} \mathrm{NO}\left([\mathrm{M}]^{+}\right)$203.1310, found 203.1303. $[\alpha]^{25}+41\left(c=0.33, \mathrm{CHCl}_{3}\right)$.

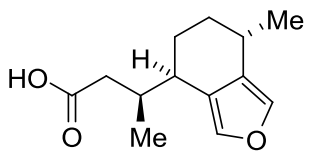

(S)-3-((4R,7S)-7-methyl-4,5,6,7-tetrahydroisobenzofuran-4-yl)butanoic acid (16). To a solution of $45 \mathrm{mg}(0.22 \mathrm{mmol}) 15 \mathrm{in} 4.0 \mathrm{~mL}$ ethylene glycol and $1.0 \mathrm{~mL} \mathrm{H}_{2} \mathrm{O}$ in a sealed tube was added $0.99 \mathrm{~g}(17.6 \mathrm{mmol}) \mathrm{KOH}$. The reaction mixture was heated to 130 ${ }^{\circ} \mathrm{C}$ for 2 hours and after 2 hours, the light yellow solution was cooled to room temperature, then diluted with $\mathrm{H}_{2} \mathrm{O}$ and extracted with EtOAc. The organic layer was extracted once more with $\mathrm{H}_{2} \mathrm{O}$ and the combined aqueous layers were then acidified with $\mathrm{HCl}(2.0 \mathrm{M})$ until the $\mathrm{pH}$ was adjusted to 4 . The resulting aqueous solution was then extracted once with EtOAc and the resulting organic layer was dried over $\mathrm{MgSO}_{4}$, filtered, and evaporated. Filtration through a silica pad (70\% EtOAc/hexanes) afforded $44 \mathrm{mg}(90 \%)$ of 16 as a pale yellow solid.

Rf: 0.10, 25\% EtOAc/hexanes. ${ }^{1} \mathrm{H}$ NMR $(600 \mathrm{MHz}): \delta 7.20(\mathrm{t}, 1 \mathrm{H}, J=1.6 \mathrm{~Hz}), 7.18(\mathrm{t}$, $1 \mathrm{H}, J=1.6 \mathrm{~Hz}), 2.76(\mathrm{~m}, 1 \mathrm{H}), 2.57(\mathrm{~m}, 1 \mathrm{H}), 2.44(\mathrm{~m}, 2 \mathrm{H}), 2.33(\mathrm{~m}, 1 \mathrm{H}), 1.90(\mathrm{~m}, 1 \mathrm{H})$, $1.78(\mathrm{~m}, 1 \mathrm{H}), 1.36(\mathrm{ddd}, 1 \mathrm{H}, J=14 \mathrm{~Hz}), 1.21(\mathrm{~m}, 1 \mathrm{H}$ and d, 3H, $J=6.7 \mathrm{~Hz}), 0.94(\mathrm{~d}, 3 \mathrm{H}$, $J=6.9 \mathrm{~Hz}) .{ }^{13} \mathrm{C}$ NMR $(150 \mathrm{MHz}): \delta 179.33,137.45,137.37,128.60,124.05,39.04$, 37.02 , 33.52, 32.53, 27.79, 23.85, 21.01, 15.27. IR: 2928, 1708, 1538, 1455, 1413, 1291, $1129,1044 \mathrm{~cm}^{-1}$. HRMS (EI) calcd for $\mathrm{C}_{13} \mathrm{H}_{18} \mathrm{O}_{3}\left([\mathrm{M}]^{+}\right)$222.1256, found 222.1245. $[\alpha]_{\mathrm{D}}^{25}+67\left(c=0.33, \mathrm{CHCl}_{3}\right)$. 


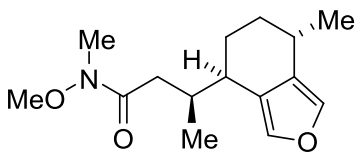

(S)- $N$-methoxy- $N$-methyl-3-((4R,7S)-7-methyl-4,5,6,7-tetrahydroisobenzofuran-4yl)butanamide (S2). A solution of $70 \mathrm{mg}(0.32 \mathrm{mmol}) \mathbf{1 6}$ in $15 \mathrm{~mL}$ DCM (anhydrous) was cooled to $0{ }^{\circ} \mathrm{C}$, and then $112 \mathrm{mg}(0.69 \mathrm{mmol})$ of 1,1 carbonyldiimidazole (CDI) was added portionwise over 70 minutes, warming to room temperature after a few minutes. The yellow solution was then cooled to $0{ }^{\circ} \mathrm{C}$, and then $61 \mathrm{mg}(0.63 \mathrm{mmol})$ of $\mathrm{N}, \mathrm{O}-$ Dimethylhydroxylamine hydrochloride was added. The solution was warmed to room temperature and after 40 minutes, another $30 \mathrm{mg}(0.31 \mathrm{mmol})$ of $\mathrm{N}, \mathrm{O}-$ Dimethylhydroxylamine hydrochloride was added. The reaction mixture was stirred for another 16 hours at room temperature, and then another $10 \mathrm{mg}$ of $\mathrm{N}, \mathrm{O}$ Dimethylhydroxylamine hydrochloride was added and after 1.5 hours, the mixture was quenched with $\mathrm{NH}_{4} \mathrm{Cl}$ (saturated) and diluted with DCM. The layers were separated and the organic layer was washed with $\mathrm{NaHCO}_{3}$ (saturated), then $\mathrm{NaCl}$ (saturated), dried over $\mathrm{MgSO}_{4}$, filtered, and evaporated. Flash column chromatography (30\% EtOAc/hexanes) afforded $79 \mathrm{mg}$ (94\%) of $\mathbf{S 2}$ as a clear, colorless oil.

Rf: $0.59,40 \%$ EtOAc/hexanes. ${ }^{1} \mathrm{H}$ NMR $(600 \mathrm{MHz}): \delta 7.21(\mathrm{t}, 1 \mathrm{H}, J=1.6 \mathrm{~Hz}), 7.17(\mathrm{t}$, $1 \mathrm{H}, J=1.6 \mathrm{~Hz}), 3.68(\mathrm{~s}, 3 \mathrm{H}), 3.19(\mathrm{~s}, 3 \mathrm{H}), 2.72(\mathrm{~m}, 1 \mathrm{H}), 2.58(\mathrm{~m}, 1 \mathrm{H}), 2.56(\mathrm{~m}, 1 \mathrm{H})$, $2.44(\mathrm{~m}, 2 \mathrm{H}), 1.90(\mathrm{~m}, 1 \mathrm{H}), 1.78(\mathrm{~m}, 1 \mathrm{H}), 1.37(\mathrm{ddd}, 1 \mathrm{H}, J=13 \mathrm{~Hz}), 1.21(\mathrm{~m}, 1 \mathrm{H}$ and $\mathrm{d}$, $3 \mathrm{H}, J=6.7 \mathrm{~Hz}), 0.90(\mathrm{~d}, 3 \mathrm{H}, J=6.8 \mathrm{~Hz}) .{ }^{13} \mathrm{C} \mathrm{NMR}(150 \mathrm{MHz}): \delta 174.07,137.53$, 137.22, 128.63, 124.43, 61.19, 37.36, 36.43, 33.04, 32.69, 27.85, 24.07, 21.05, 15.41. IR: 2929, 1666, 1455, 1378, 1177, 1128, $1042 \mathrm{~cm}^{-1}$. HRMS (EI) calcd for $\mathrm{C}_{15} \mathrm{H}_{23} \mathrm{NO}_{3}$ $\left([\mathrm{M}]^{+}\right) 265.1678$, found 265.1674. $[\alpha]^{25}+10\left(c=0.60, \mathrm{CHCl}_{3}\right)$.

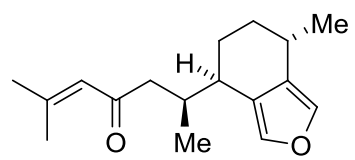

(S)-2-methyl-6-((4R,7S)-7-methyl-4,5,6,7-tetrahydroisobenzofuran-4-yl)hept-2-en-4one (17). A solution of $200 \mathrm{mg} \mathbf{S} 2(0.75 \mathrm{mmol})$ in $50 \mathrm{~mL}$ THF was cooled to $0{ }^{\circ} \mathrm{C}$ and then $4.0 \mathrm{~mL}(3.0 \mathrm{mmol})$ of a $0.5 \mathrm{M} 2$-methyl-1-propenylmagnesium bromide solution in THF was added dropwise. The reaction mixture was warmed to room temperature for 10 minutes, then quenched with a saturated aqueous solution of $\mathrm{NaHCO}_{3}$. The layers were separated and the organic layer was washed with $\mathrm{NaCl}$ (saturated), dried over $\mathrm{MgSO}_{4}$, filtered, and evaporated. Flash column chromatography (5\% EtOAc/hexanes) afforded $169 \mathrm{mg}(86 \%)$ of $\mathbf{1 7}$ as a clear, colorless oil that was unstable in $\mathrm{CHCl}_{3}$ and $\mathrm{CDCl}_{3}$.

Rf: $0.59,10 \%$ EtOAc/hexanes. ${ }^{1} \mathrm{H}$ NMR $\left(600 \mathrm{MHz}, \mathrm{C}_{6} \mathrm{D}_{6}\right): \delta 7.10(\mathrm{t}, 1 \mathrm{H}, J=1.6 \mathrm{~Hz})$, $7.09(\mathrm{t}, 1 \mathrm{H}, J=1.1 \mathrm{~Hz}), 5.83$ (quintet, $1 \mathrm{H}, J=1.3 \mathrm{~Hz}), 2.63(\mathrm{~m}, 1 \mathrm{H}), 2.57(\mathrm{~m}, 1 \mathrm{H}), 2.40$ (m, 1H), $2.27(\mathrm{dd}, 1 \mathrm{H}, J=15.8 \mathrm{~Hz}), 2.18(\mathrm{dd}, 1 \mathrm{H}, J=15.8 \mathrm{~Hz}), 2.14(\mathrm{~d}, 3 \mathrm{H}, J=1.1 \mathrm{~Hz})$, $1.64(\mathrm{~m}, 1 \mathrm{H}), 1.54(\mathrm{~m}, 1 \mathrm{H}), 1.49(\mathrm{~d}, 3 \mathrm{H}, J=1.3 \mathrm{~Hz}), 1.18(\mathrm{ddd}, 1 \mathrm{H}, J=12.9 \mathrm{~Hz}), 1.05$ 
(m, $1 \mathrm{H}$ and d, $3 \mathrm{H}, J=6.7 \mathrm{~Hz}), 0.90(\mathrm{~d}, 3 \mathrm{H}, J=6.8 \mathrm{~Hz}) .{ }^{13} \mathrm{C} \mathrm{NMR}\left(100 \mathrm{MHz}, \mathrm{C}_{6} \mathrm{D}_{6}\right)$ ) $\delta 198.65,153.43,137.56,137.24,128.39,124.29,124.05,48.73,37.21,27.83,26.82$, 24.28, 20.74, 20.17, 15.47. IR: 2928, 2367, 1686, 1633, 1447, 1377, 1266, 1129, 1043 $\mathrm{cm}^{-1}$. HRMS (EI) calcd for $\mathrm{C}_{17} \mathrm{H}_{23} \mathrm{O}_{2}\left([\mathrm{M}-\mathrm{H}]^{-}\right) 259.1698$, found 259.1704 .

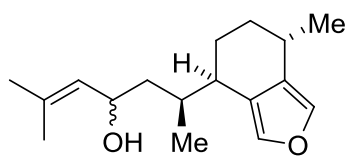

(6S)-2-methyl-6-((4R,7S)-7-methyl-4,5,6,7-tetrahydroisobenzofuran-4-yl)hept-2-en4-ol (8). A solution of $167 \mathrm{mg}(0.64 \mathrm{mmol}) \mathbf{1 7}$ in $35.0 \mathrm{~mL}$ DCM (anhydrous) was cooled to $-40{ }^{\circ} \mathrm{C}$ and then $8.0 \mathrm{~mL}(3.2 \mathrm{mmol})$ of a freshly prepared $0.4 \mathrm{M} \mathrm{CeCl}_{3}$ solution in $\mathrm{MeOH}$ was added dropwise. The reaction mixture was stirred for 10 minutes before 121 mg $(3.2 \mathrm{mmol})$ of $\mathrm{NaBH}_{4}$ was added, and then the mixture was allowed to gradually warm to $-25^{\circ} \mathrm{C}$ over 50 minutes at which point it was quenched slowly with a saturated aqueous solution of $\mathrm{NaHCO}_{3}$. The layers were separated and the organic layer was dried over $\mathrm{MgSO}_{4}$, filtered, and evaporated. Flash column chromatography $(10 \%$ EtOAc/hexanes) afforded $159 \mathrm{mg}(95 \%)$ of $\mathbf{8}$ as a clear, colorless oil that was a mixture of diastereomers.

Rf: $0.21,10 \%$ EtOAc/hexanes. ${ }^{1} \mathrm{H}$ NMR (600 MHz): $\delta 7.16(\mathrm{t}, 1 \mathrm{H}, J=1.6 \mathrm{~Hz}), 7.14(\mathrm{dd}$, $1 \mathrm{H}, J=3.4 \mathrm{~Hz}), 5.19(\mathrm{~m}, 0.6 \mathrm{H}), 5.18(\mathrm{~m}, 0.4 \mathrm{H}), 4.47(\mathrm{~m}, 1 \mathrm{H}), 2.76(\mathrm{~m}, 0.4 \mathrm{H}), 2.67(\mathrm{~m}$, $0.6 \mathrm{H}), 2.56(\mathrm{~m}, 1 \mathrm{H}), 2.07(\mathrm{~m}, 0.6 \mathrm{H}), 1.88(\mathrm{~m}, 1.4 \mathrm{H}), 1.73(2$ sets of dd, $6 \mathrm{H}, J=1.3 \mathrm{~Hz})$, $1.62(\mathrm{br} \mathrm{m}, 1.4 \mathrm{H}), 1.59(\mathrm{~m}, 0.6 \mathrm{H}), 1.33(\mathrm{~m}, 2 \mathrm{H}), 1.25(\mathrm{t}, 1 \mathrm{H} J=3.4 \mathrm{~Hz}), 1.20(\mathrm{~d}, 3 \mathrm{H}, J=$ $6.7 \mathrm{~Hz}), 1.15(\mathrm{~m}, 1 \mathrm{H}), 0.85(2$ sets of d, $3 \mathrm{H}, J=6.9 \mathrm{~Hz}) .{ }^{13} \mathrm{C}$ NMR $\left(100 \mathrm{MHz}, \mathrm{CDCl}_{3}\right)$ : $\delta 135.56,134.77,67.14,66.74,42.37,42.33,37.68,37.38,32.94,32.88,32.57,27.94$, 25.81, 25.75, 23.82, 23.74, 21.02, 18.24, 18.16, 15.69, 15.04. IR: 3358, 2959, 2361, $1672,1538,1449,1376,1265,1129,1044 \mathrm{~cm}^{-1}$. HRMS (EI) calcd for $\mathrm{C}_{17} \mathrm{H}_{26} \mathrm{O}_{2}\left([\mathrm{M}]^{+}\right)$ 262.1933 , found 262.1926 .

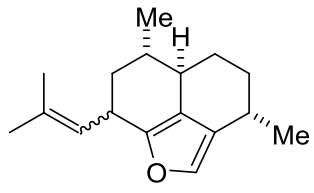

(3S,5aR,6S)-3,6-dimethyl-8-(2-methylprop-1-en-1-yl)-4,5,5a,6,7,8-hexahydro-3Hnaphtho[1,8-bc]furan (18). To a solution of $22 \mathrm{mg} 8(0.084 \mathrm{mmol})$ in $6 \mathrm{~mL} \mathrm{MeNO}_{2}$ was added $1.5 \mathrm{~mL}$ of a freshly prepared solution of $1 \mathrm{mg} / \mathrm{mL} \mathrm{La(OTf})_{3}$ in $\mathrm{MeNO}_{2}$ over 5 minutes. The pink reaction mixture was stirred for 15 minutes until it was quenched with a saturated aqueous solution of $\mathrm{NaHCO}_{3}$ and extracted with EtOAc. The layers were separated and the organic layer was washed with $\mathrm{NaHCO}_{3}$ (saturated) until no pink color remained. It was then washed with $\mathrm{NaCl}$ (saturated), dried over $\mathrm{MgSO}_{4}$, filtered, and evaporated. Flash column chromatography (0.1 to 2 to $5 \%$ EtOAc/hexanes) afforded 
$14 \mathrm{mg}(70 \%)$ of $\mathbf{1 8}$ as a clear, colorless oil that was a 0.4:0.6 mixture of diastereomers at the position of ring closure. (Inseparable by flash column chromatography or HPLC).

Rf: 0.48, hexanes. ${ }^{1} \mathrm{H}$ NMR (400 MHz, $\left.\mathrm{C}_{6} \mathrm{D}_{6}\right): \delta 7.08(\mathrm{dd}, 0.4 \mathrm{H}, J=2.6 \mathrm{~Hz}), 7.07(\mathrm{dd}$, $0.6 \mathrm{H}, J=2.5 \mathrm{~Hz}), 5.31(\mathrm{dq}, 0.4 \mathrm{H}, J=13.8 \mathrm{~Hz}), 5.23(\mathrm{dq}, 0.6 \mathrm{H}, J=13.7 \mathrm{~Hz}), 3.73(\mathrm{~m}$, $1 \mathrm{H}), 2.57(\mathrm{~m}, 1 \mathrm{H}), 1.96(\mathrm{br} \mathrm{m}, 0.6 \mathrm{H}), 1.83(\mathrm{~m}, 2.4 \mathrm{H}), 1.70(\mathrm{~m}, 6 \mathrm{H}), 1.47(\mathrm{~m}, 0.6 \mathrm{H}), 1.34$ $(\mathrm{m}, 1 \mathrm{H}), 1.30(\mathrm{~m}, 1.4 \mathrm{H}), 1.16$ (doublet with shoulder, $3 \mathrm{H}, J=10.2 \mathrm{~Hz}), 1.10(\mathrm{br} \mathrm{m}, 2 \mathrm{H})$, 0.91 (d with shoulder, $3 \mathrm{H}, J=9.5 \mathrm{~Hz}$ ). ${ }^{13} \mathrm{C}$ NMR $\left(100 \mathrm{MHz}, \mathrm{C}_{6} \mathrm{D}_{6}\right): \delta 150.30,136.52$, $136.42,132.44,120.98,109.90,40.86,40.28,39.99,39.82,36.10,35.42,33.74,33.63$, $33.22,32.19,28.33,28.26,27.37,25.56,25.43,21.72,18.16,18.03,17.77,17.66$. IR: 2955, 2361, 1650, 1550, 1452, 1375, 1309, 1256, 1130, $1088 \mathrm{~cm}^{-1}$. HRMS (EI) calcd for $\mathrm{C}_{17} \mathrm{H}_{24} \mathrm{O}\left([\mathrm{M}]^{+}\right)$244.1827, found 244.1839.

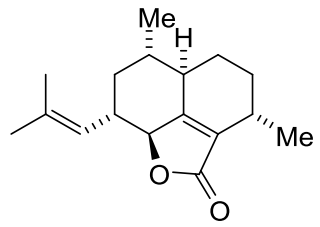

(3S,5aR,6S,8S,8aS)-3,6-dimethyl-8-(2-methylprop-1-en-1-yl)-3,4,5,5a,6,7,8,8aoctahydro-2H-naphtho[1,8-bc]furan-2-one, amphilectolide (1). To a solution of $30 \mathrm{mg}$ $(0.12 \mathrm{mmol}) 18$ in $6 \mathrm{~mL}$ DCM was added $6 \mathrm{mg}(0.006 \mathrm{mmol})$ Rose Bengal, $3 \mathrm{~mL} \mathrm{MeOH}$, and $0.10 \mathrm{~mL}(0.60 \mathrm{mmol})$ DIEA. The solution was cooled to $-78^{\circ} \mathrm{C}$ and irradiated with a UV lamp (Replux Belgium RL $160 \mathrm{~W}, 225$ - 235 Volts). $\mathrm{O}_{2}$ was bubbled through for 15 minutes, then the reaction mixture was quickly evaporated at $30{ }^{\circ} \mathrm{C}$, taken up in $6 \mathrm{~mL}$ EtOH; $23 \mathrm{mg}(0.60 \mathrm{mmol})$ of $\mathrm{NaBH}_{4}$ was then added. After 10 minutes, the reaction mixture was quenched with a saturated aqueous solution of $\mathrm{NaHCO}_{3}$, diluted with $\mathrm{Et}_{2} \mathrm{O}$, washed with $\mathrm{NaCl}$ (saturated), dried over $\mathrm{MgSO}_{4}$, filtered, and evaporated. Flash column chromatography $\left(6 \% \mathrm{Et}_{2} \mathrm{O} /\right.$ hexanes followed by $5 \%$ EtOAc/hexanes) afforded $3.3 \mathrm{mg}$ $(11 \%)(\mathbf{1})$ as a white solid.

\begin{tabular}{cccc}
\hline${ }^{\mathrm{I}} \mathrm{H}$ NMR isolation & ${ }^{\mathrm{I}} \mathrm{H}$ NMR current & ${ }^{13} \mathrm{C}$ NMR isolation & ${ }^{13} \mathrm{C}$ NMR current \\
\hline $5.07, \mathrm{br} \mathrm{dd}(1.2,9.0)$ & $5.07, \mathrm{dt}(1.4,9.0)$ & 172.9 & 172.93 \\
$4.35, \mathrm{~d}(10.5)$ & $4.35, \mathrm{~d}(9.0)$ & 165.0 & 165.05 \\
$2.42, \mathrm{~m}$ & $2.42, \mathrm{~m}$ & 134.5 & 134.53 \\
$2.23, \mathrm{~m}$ & $2.24, \mathrm{~m}$ & 128.1 & 128.11 \\
$2.16, \mathrm{~m}$ & $2.17, \mathrm{~m}$ & 125.2 & 125.15 \\
$2.01, \mathrm{~m}$ & $2.05, \mathrm{~m}$ & 83.6 & 83.64 \\
$1.86, \mathrm{~m}$ & $1.91, \mathrm{~m}$ & 44.1 & 44.11 \\
$1.72, \mathrm{br} \mathrm{s}$ & $1.72, \mathrm{~d}(1.44)$ & 41.0 & 40.95 \\
$1.62, \mathrm{brd}(0.9)$ & $1.63 \mathrm{~d}(1.38)$ & 39.5 & 39.51 \\
$1.56, \mathrm{~m}$ & $1.56, \mathrm{~m}$ & 38.4 & 38.36 \\
$1.23, \mathrm{~d}(7.2)$ & $1.23, \mathrm{~d}(8.0)$ & 31.2 & 31.19 \\
$1.20, \mathrm{~m}$ & $1.20, \mathrm{~m}$ & 27.3 & 27.32 \\
$1.13, \mathrm{~m}$ & $1.13, \mathrm{~m}$ & 27.2 & 27.17 \\
\hline \multicolumn{4}{c}{}
\end{tabular}




\begin{tabular}{cccc}
\hline $1.11, \mathrm{~m}$ & $1.11, \mathrm{~m}$ & 25.8 & 25.85 \\
$1.06, \mathrm{~m}$ & $1.06, \mathrm{~m}$ & 19.1 & 19.12 \\
$1.04, \mathrm{~d}(7.2)$ & $1.04, \mathrm{~d}(6.5)$ & 18.3 & 18.33 \\
& & 17.8 & 17.83 \\
\hline
\end{tabular}

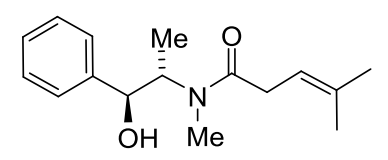

$N$-((1S,2S)-1-hydroxy-1-phenylpropan-2-yl)- $N$,4-dimethylpent-3-enamide (20). A solution of $1.16 \mathrm{~g}(10.2 \mathrm{mmol}) 19$ and $2.5 \mathrm{~mL}(18.2 \mathrm{mmol})$ triethylamine (TEA) in $20 \mathrm{~mL}$ $\mathrm{MeCN}$ was cooled to $0{ }^{\circ} \mathrm{C}$, and then $1.9 \mathrm{~mL}(15.3 \mathrm{mmol})$ of pivaloyl chloride was added. To the resulting white slurry was added $5 \mathrm{~mL}$ THF to enhance solubility. The reaction mixture turned yellow, and after 20 minutes, a solution of $1.7 \mathrm{~g}(10.2 \mathrm{mmol})$ of $(+)$ pseudoephedrine and $1.4 \mathrm{~mL}(10.2 \mathrm{mmol})$ of TEA in $15 \mathrm{~mL}$ THF was added. The reaction mixture was warmed to room temperature and stirred for another 75 minutes, at which point it was quenched with water. The volatiles were removed by rotary evaporation, and then a solution of $\mathrm{NaOH}(0.5 \mathrm{M})$ was added. This solution was extracted with a mixture of $10 \%$ methanol in DCM twice, and the resulting organic layer was washed with a $1.0 \mathrm{M} \mathrm{NaOH}$ solution. The organic layer was then dried over $\mathrm{MgSO}_{4}$, filtered, and evaporated and the crude oil purified by flash column chromatography $(60 \%$ EtOAc/hexanes) to provide $2.04 \mathrm{~g}(76 \%) 20$ as a white solid that was a 1:2 mixture of rotamers.

Rf: $0.31,60 \%$ EtOAc/hexanes. ${ }^{1} \mathrm{H}$ NMR $(300 \mathrm{MHz})$ of major rotamer: $\delta 7.32(\mathrm{~m}, 5 \mathrm{H})$, $5.21(\mathrm{t}, 1 \mathrm{H}, J=7.1 \mathrm{~Hz}), 4.60(\mathrm{dd}, 1 \mathrm{H}, J=7.7 \mathrm{~Hz}), 4.48(\mathrm{br} \mathrm{s}, 1 \mathrm{H}), 4.39(\mathrm{~m}, 1 \mathrm{H}), 3.03$ (d, $2 \mathrm{H}, J=6.7 \mathrm{~Hz}), 2.79(\mathrm{~s}, 3 \mathrm{H}), 1.74(\mathrm{~s}, 3 \mathrm{H}), 1.63(\mathrm{~s}, 3 \mathrm{H}), 1.13(\mathrm{~d}, 3 \mathrm{H}, J=7.0 \mathrm{~Hz}) .{ }^{13} \mathrm{C}$ NMR $(75 \mathrm{MHz})$ of major rotamer: $\delta 174.35,142.48,134.92,128.30,127.56,126.38$, $116.58,75.42,58.62,34.56,33.17,25.69,18.06,14.39$. IR: 3373, 2916, 2363, 1633, $1453,1403,1262,1115 \mathrm{~cm}^{-1}$. HRMS (EI) calcd for $\mathrm{C}_{16} \mathrm{H}_{24} \mathrm{NO}_{2}\left([\mathrm{M}+\mathrm{H}]^{+}\right)$262.1807, found 262.1802. $[\alpha]^{25}+116\left(c=0.38, \mathrm{CHCl}_{3}\right)$.

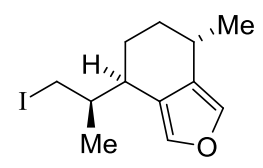

(4R,7S)-4-((R)-1-iodopropan-2-yl)-7-methyl-4,5,6,7-tetrahydroisobenzofuran (22).

To a solution of $4.03 \mathrm{~g}(14.8 \mathrm{mmol}) \mathbf{2 1}$ in $200 \mathrm{~mL}$ acetone (anhydrous) in a pressure tube was added $11.1 \mathrm{~g}(74.0 \mathrm{mmol})$ of NaI. The mixture was excluded from light and heated to $85{ }^{\circ} \mathrm{C}$ for 2.5 hours. The mixture was left to come to room temperature, then quenched with $\mathrm{H}_{2} \mathrm{O}$ and diluted with $\mathrm{Et}_{2} \mathrm{O}$. The layers were separated and the organic layer was washed with $\mathrm{NaCl}$ (saturated), dried over $\mathrm{Na}_{2} \mathrm{SO}_{4}$, filtered, and evaporated. Flash column chromatography (2\% EtOAc/hexanes) afforded $3.78 \mathrm{~g}(84 \%)$ of 22 as a light-sensitive oil. 
Rf: 0.38 , hexanes. ${ }^{1} \mathrm{H}$ NMR $(400 \mathrm{MHz}): \delta 7.18(\mathrm{~d}, 1 \mathrm{H}, J=1.6 \mathrm{~Hz}), 7.16(\mathrm{~d}, 1 \mathrm{H}, J=1.6$ $\mathrm{Hz}), 3.25(\mathrm{dd}, 2 \mathrm{H}, J=7.0 \mathrm{~Hz}), 2.96(\mathrm{~m}, 1 \mathrm{H}), 2.56(\mathrm{~m}, 1 \mathrm{H}), 2.12(\mathrm{~m}, 1 \mathrm{H}), 1.89(\mathrm{~m}, 1 \mathrm{H})$, $1.73(\mathrm{~m}, 1 \mathrm{H}), 1.25(\mathrm{~m}, 5 \mathrm{H}), 0.99(\mathrm{~d}, 3 \mathrm{H}, J=6.9 \mathrm{~Hz}) .{ }^{13} \mathrm{C} \mathrm{NMR}(75 \mathrm{MHz}): \delta 137.46$, 137.31, 128.56, 124.04, 39.63, 36.68, 32.25, 27.72, 23.14, 21.10, 15.98, 13.42. IR: 2854, 1455, 1376, 1194, $1045 \mathrm{~cm}^{-1}$. HRMS (EI) calcd for $\mathrm{C}_{12} \mathrm{H}_{17} \mathrm{IO}\left([\mathrm{M}]^{+}\right) 304.0324$, found 304.0322. $[\alpha]^{25}+31\left(c=0.37, \mathrm{CHCl}_{3}\right)$.

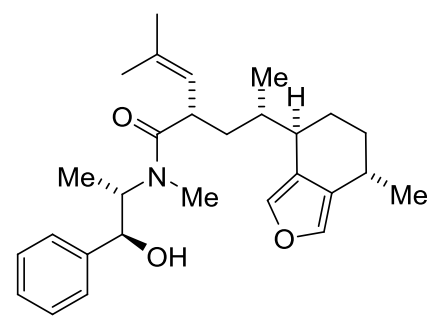

$(S)-N-((1 S, 2 S)-1-h y d r o x y-1-p h e n y l p r o p a n-2-y l)-N, 4-d i m e t h y l-2-((S)-2-((4 R, 7 S)-7-$ methyl-4,5,6,7-tetrahydroisobenzofuran-4-yl)propyl)pent-3-enamide (23). A suspension of $3.14 \mathrm{~mL}(2.25 \mathrm{~g}, 22.2 \mathrm{mmol})$ diisopropylamine and $3.56 \mathrm{~g}(84.0 \mathrm{mmol})$ $\mathrm{LiCl}$ in $20 \mathrm{~mL}$ THF was cooled to $-78{ }^{\circ} \mathrm{C}$, and $13.5 \mathrm{~mL}(21.6 \mathrm{mmol})$ of $n \mathrm{BuLi}(1.6 \mathrm{M}$ in hexanes) were added dropwise. The resulting reaction mixture was warmed to $0{ }^{\circ} \mathrm{C}$ for 5 minutes, and then cooled to $-78{ }^{\circ} \mathrm{C}$. A solution of $2.82 \mathrm{mg}(10.8 \mathrm{mmol})$ of chiral auxiliary 20 in $35 \mathrm{~mL}$ THF cooled to $0{ }^{\circ} \mathrm{C}$ was then added dropwise by cannula. The reaction mixture turned bright yellow. The reaction mixture was stirred for 1 hour at -78 ${ }^{\circ} \mathrm{C}$, and was then warmed to $0{ }^{\circ} \mathrm{C}$ for 15 minutes, then to room temperature for 5 minutes. The reaction mixture was cooled to $0{ }^{\circ} \mathrm{C}$, and a solution of $1.83 \mathrm{~g}(6.02 \mathrm{mmol})$ of iodide 22 was added dropwise. The reaction mixture was warmed to room temperature and the flask was covered with foil to protect it from light. After 19 hours at room temperature, the reaction mixture was quenched with a 1:1 solution of water: $\mathrm{NH}_{4} \mathrm{Cl}$ (saturated) and the resulting mixture was extracted four times with EtOAc. The combined organic extracts were dried over $\mathrm{Na}_{2} \mathrm{SO}_{4}$, evaporated and the crude oil was purified by flash column chromatography (10 to $60 \%$ EtOAc/hexanes) to provide $2.51 \mathrm{~g}(95 \%) 23$ as a yellow oil that was a 2:1 mixture of rotamers. Excess chiral auxiliary $\mathbf{2 0}$ was also recovered $(815 \mathrm{mg})$.

Rf: $0.39,40 \%$ EtOAc/hexanes. ${ }^{1} \mathrm{H}$ NMR $(600 \mathrm{MHz})$ of major rotamer: $\delta 7.35(\mathrm{~m}, 5 \mathrm{H})$, $7.18(\mathrm{~s}, 1 \mathrm{H}), 7.12(\mathrm{~s}, 1 \mathrm{H}), 5.10(\mathrm{~d}, 1 \mathrm{H}, J=9.7 \mathrm{~Hz}), 4.64(\mathrm{~m}, 1 \mathrm{H}), 4.38(\mathrm{br} \mathrm{s}, 1 \mathrm{H}), 3.78$ $(\mathrm{m}, 1 \mathrm{H}), 3.41(\mathrm{~m}, 1 \mathrm{H}), 2.81(\mathrm{~s}, 3 \mathrm{H}), 2.67(\mathrm{~m}, 1 \mathrm{H}), 2.58(\mathrm{~m}, 1 \mathrm{H}), 1.90(\mathrm{~m}, 2 \mathrm{H}), 1.74(\mathrm{~m}$, $2 \mathrm{H}), 1.66(\mathrm{~d}, 1 \mathrm{H}, J=4.5 \mathrm{~Hz}), 1.37(\mathrm{~m}, 2 \mathrm{H}), 1.20(\mathrm{~m}, 9 \mathrm{H}), 0.88(\mathrm{~m}, 3 \mathrm{H}), 0.81(\mathrm{~d}, 3 \mathrm{H}, J=$ $6.9 \mathrm{~Hz}) .{ }^{13} \mathrm{C}$ NMR $(150 \mathrm{MHz})$ of major rotamer: $\delta 176.89,142.52,137.22,137.16$, $133.34,128.84,128.70,128.25,127.47,126.77,126.23,124.80,123.66,76.50,57.87$, $40.93,37.38,37.33,33.85,32.89,27.99,27.94,25.70,23.77,21.00,18.15,15.63,14.40$. IR: 3376, 2929, 2871, 2361, 1628, 1452, 1405, 1264, 1127, $1044 \mathrm{~cm}^{-1}$. HRMS (EI) calcd for $\mathrm{C}_{28} \mathrm{H}_{39} \mathrm{NO}_{3}\left([\mathrm{M}]^{+}\right)$437.2930, found 437.2931. $[\alpha]^{25}{ }_{\mathrm{D}}+117\left(c=0.40, \mathrm{CHCl}_{3}\right)$. 


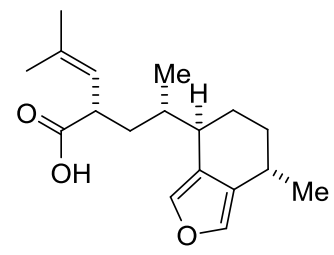

(S)-4-methyl-2-((S)-2-((4R,7S)-7-methyl-4,5,6,7-tetrahydroisobenzofuran-4-

yl)propyl)pent-3-enoic acid (9). To $2.51 \mathrm{~g}(5.74 \mathrm{mmol})$ of compound $\mathbf{2 3}$ were added 20 $\mathrm{mL}$ of $t \mathrm{BuOH}, 57.4 \mathrm{~g}$ of an aqueous tetra- $n$-butylammonium hydroxide solution $(40 \%$ $\mathrm{w} / \mathrm{w}, 28.7 \mathrm{mmol}$ ) and $50 \mathrm{~mL}$ of water and the mixture was heated to $100{ }^{\circ} \mathrm{C}$ at reflux for 20 hours. After cooling the reaction mixture to room temperature, it was partitioned between a $0.5 \mathrm{M}$ solution of $\mathrm{NaOH}$ and EtOAc. The layers were separated, and the aqueous layer was extracted with EtOAc twice. The $\mathrm{pH}$ of the aqueous layer was adjusted to $\mathrm{pH}=1$ by addition of a $0.5 \mathrm{M} \mathrm{HCl}$ aqueous solution. The aqueous layer was then extracted three more times with EtOAc. The combined organic layers were washed with water, dried over $\mathrm{Na}_{2} \mathrm{SO}_{4}$, filtered, and evaporated. The crude material was purified using flash column chromatography $(40 \% \mathrm{EtOAc} /$ hexanes and $1 \% \mathrm{AcOH})$ and the fractions containing product were washed with $\mathrm{NaHCO}_{3}$ (saturated), then dried over $\mathrm{Na}_{2} \mathrm{SO}_{4}$, filtered, and evaporated to afford $1.66 \mathrm{~g}(99 \%)$ of $\mathbf{9}$ as a pale yellow oil.

Rf: 0.46, 60\% EtOAc/Hexanes. ${ }^{1} \mathrm{H}$ NMR (600 MHz): $\delta 7.16(\mathrm{t}, 1 \mathrm{H}, J=1.6 \mathrm{~Hz}), 7.09(\mathrm{t}$, $1 \mathrm{H}, J=1.5 \mathrm{~Hz}), 5.13(\mathrm{~d}, 1 \mathrm{H} J=9.5 \mathrm{~Hz}), 3.38(\mathrm{~m}, 1 \mathrm{H}), 2.67(\mathrm{~m}, 1 \mathrm{H}), 2.55(\mathrm{~m}, 2 \mathrm{H}), 1.87$ $(\mathrm{m}, 3 \mathrm{H}), 1.74$ (d with shoulder, $4 \mathrm{H} J=1.3 \mathrm{~Hz}), 1.69(\mathrm{~d}, 3 \mathrm{H} J=1.4 \mathrm{~Hz}), 1.43(\mathrm{~m}, 1 \mathrm{H})$, $1.31(\mathrm{~m}, 1 \mathrm{H}), 1.20(\mathrm{~d}, 3 \mathrm{H}, J=6.7 \mathrm{~Hz}), 1.17(\mathrm{~m}, 1 \mathrm{H}), 0.86(\mathrm{~d}, 3 \mathrm{H}, J=6.7 \mathrm{~Hz}) .{ }^{13} \mathrm{C} \mathrm{NMR}$ (150 MHz): $\delta 181.04,137.22,137.18,135.51,128.79,124.71,122.24,42.85,37.22$, $36.70,33.98,32.85,27.93,25.77,23.54,20.99,18.21,15.52$. IR: 2959, 2926, 1710, $1448,1377,1292,1130,1044 \mathrm{~cm}^{-1}$. HRMS (EI) calcd for $\mathrm{C}_{18} \mathrm{H}_{26} \mathrm{O}_{3}\left([\mathrm{M}]^{+}\right)$290.1882, found 290.1883. $[\alpha]^{25}+78\left(c=0.63, \mathrm{CHCl}_{3}\right)$.

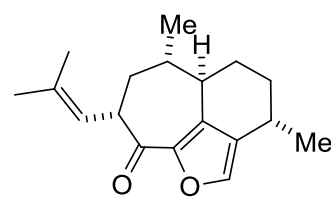

(3S,5aR,6S,8S)-3,6-dimethyl-8-(2-methylprop-1-en-1-yl)-4,5,5a,6,7,8-

hexahydrocyclohepta[cd]isobenzofuran-9(3H)-one (24). A solution of $300 \mathrm{mg}(1.03$ mmol) 9 in $200 \mathrm{~mL} \mathrm{DCM}$ was cooled to $0{ }^{\circ} \mathrm{C}$, and then $0.201 \mathrm{~mL}$ (303 mg, $1.44 \mathrm{mmol}$ ) of trifluoroacetic anhydride was added using a teflon cannula. The reaction mixture was warmed to room temperature, and after 10 minutes, $2.06 \mathrm{~mL}$ of a $1.0 \mathrm{M}$ solution of $\mathrm{ZnCl}_{2}$ ( $2.06 \mathrm{mmol})$ in THF was added dropwise. The pale yellow reaction mixture was stirred at room temperature for 30 minutes, then warmed to $40{ }^{\circ} \mathrm{C}$ for 1 hour. The reaction mixture was then quenched with an aqueous solution of $1.0 \mathrm{M} \mathrm{HCl}$, the layers were 
separated, and the organic layer was subsequently washed with a saturated aqueous solution of $\mathrm{NaHCO}_{3}$ and $\mathrm{NaCl}$ (saturated). The organic layers were then dried over $\mathrm{Na}_{2} \mathrm{SO}_{4}$, filtered, and evaporated. The crude material was purified using flash column chromatography (10\% EtOAc/hexanes) to afford $200 \mathrm{mg}$ (71\%) of $\mathbf{2 4}$ as a crystalline white solid.

Rf: $0.58,25 \%$ EtOAc/hexanes. ${ }^{1} \mathrm{H}$ NMR (600 MHz): $\delta 7.38(\mathrm{~d}, 1 \mathrm{H}, J=1.6 \mathrm{~Hz}), 5.28$ $(\mathrm{dm}, 1 \mathrm{H} J=8.7 \mathrm{~Hz}), 3.47(\mathrm{~m}, 1 \mathrm{H}), 2.65(\mathrm{~m}, 1 \mathrm{H}), 2.35(\mathrm{~m}, 1 \mathrm{H}), 2.13(\mathrm{~m}, 1 \mathrm{H}), 2.03(\mathrm{~m}$, $1 \mathrm{H}), 1.86(\mathrm{~m}, 3 \mathrm{H}), 1.78(\mathrm{~s}, 3 \mathrm{H}), 1.65(\mathrm{~s}, 3 \mathrm{H}), 1.24(\mathrm{~m}$ with d, $5 \mathrm{H} J=6.6 \mathrm{~Hz}), 1.13(\mathrm{~d}, 3 \mathrm{H}$, $J=6.1 \mathrm{~Hz}) .{ }^{13} \mathrm{C}$ NMR $(150 \mathrm{MHz}): \delta 190.36,147.51,142.16,134.31,133.74,129.52$, $123.73,50.20,43.61,41.37,38.66,32.90,28.68,27.25,25.78,21.59,21.23,18.12$. IR: $2955,2923,1650,1525,1442,1400,1284 \mathrm{~cm}^{-1}$. HRMS (EI) calcd for $\mathrm{C}_{18} \mathrm{H}_{24} \mathrm{O}_{2}\left([\mathrm{M}]^{+}\right)$ 272.1776, found 272.1773. $[\alpha]^{25}{ }_{\mathrm{D}}+14\left(c=0.30, \mathrm{CHCl}_{3}\right)$.

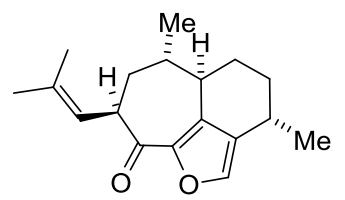

$(3 S, 5 \mathrm{a} R, 6 S, 8 R)$-3,6-dimethyl-8-(2-methylprop-1-en-1-yl)-4,5,5a,6,7,8-

hexahydrocyclohepta[cd]isobenzofuran-9(3H)-one (25). To a solution of $17 \mathrm{mg}$ $(0.062 \mathrm{mmol}) 24$ in $2 \mathrm{~mL} \mathrm{PhCH}_{3}$ was added $0.028 \mathrm{~mL}$ DBU $(0.19 \mathrm{mmol})$ and the solution was stirred at room temperature for 48 hours, then concentrated and purified by flash column chromatography (10\% EtOAc/hexanes) to obtain $17 \mathrm{mg}$ (quantitative yield) of $\mathbf{2 5}$ as a yellow oil.

${ }^{1} \mathrm{H}$ NMR (600 MHz): $\delta 7.38(\mathrm{~d}, 1 \mathrm{H}, J=1.5 \mathrm{~Hz}), 5.43(\mathrm{~d}, 1 \mathrm{H} J=9.1 \mathrm{~Hz}), 3.45(\mathrm{~m}, 1 \mathrm{H})$, $2.62(\mathrm{~m}, 1 \mathrm{H}), 2.46(\mathrm{~m}, 1 \mathrm{H}), 2.22(\mathrm{~m}, 1 \mathrm{H}), 1.94(\mathrm{~m}, 2 \mathrm{H}), 1.79(\mathrm{~m}, 1 \mathrm{H}), 1.77(\mathrm{~s}, 3 \mathrm{H}), 1.62$ $(\mathrm{s}, 3 \mathrm{H}), 1.61(\mathrm{~m}, 1 \mathrm{H}), 1.30(\mathrm{~m}, 1 \mathrm{H}), 1.24(\mathrm{~m}$ with d, $4 \mathrm{H} J=6.7 \mathrm{~Hz}), 1.12(\mathrm{~d}, 3 \mathrm{H}, J=6.7$ Hz). ${ }^{13} \mathrm{C}$ NMR $(150 \mathrm{MHz}): \delta 190.26,147.79,141.60,135.49,133.39,130.02,122.44$, $46.13,39.97,39.61,35.81,32.61,29.46,27.68,25.99,20.76,20.59,18.11$.

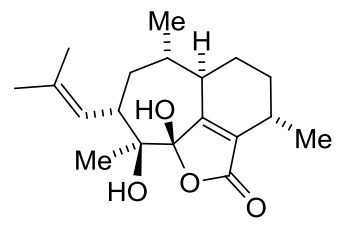

$(3 S, 5 \mathrm{a} R, 6 S, 8 S, 9 \mathrm{~S}, 9 \mathrm{a} S)$-9,9a-dihydroxy-3,6,9-trimethyl-8-(2-methylprop-1-en-1-yl)-

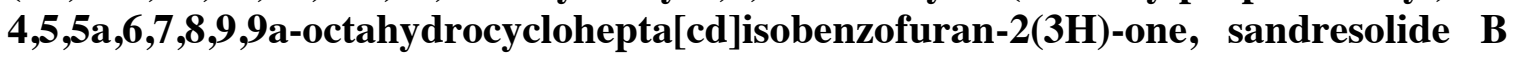
(3). A solution of $30 \mathrm{mg}(0.11 \mathrm{mmol})$ of 9 in $12 \mathrm{~mL}$ THF was cooled to $-78^{\circ} \mathrm{C}$, and then $0.147 \mathrm{~mL}(0.44 \mathrm{mmol})$ of a $3.0 \mathrm{M}$ solution of methylmagnesium bromide in $\mathrm{Et}_{2} \mathrm{O}$ was added. After 10 minutes at $-78{ }^{\circ} \mathrm{C}$, the reaction mixture was left to come to room temperature over 20 minutes and was then quenched with a saturated aqueous solution of $\mathrm{NaHCO}_{3}$. The layers were separated, and the aqueous layer was extracted twice with 
$\mathrm{Et}_{2} \mathrm{O}$. The combined organic layers were washed with $\mathrm{NaCl}$ (saturated), dried over $\mathrm{Na}_{2} \mathrm{SO}_{4}$, filtered, and evaporated. Crude material was taken on directly due to instability and was dissolved in $8 \mathrm{~mL}$ DCM. To this solution was added one spatula edge of tetraphenylporphyrine. The solution was then cooled to $-78^{\circ} \mathrm{C}$, and oxygen was bubbled through while the flask was irradiated with a UV lamp (Replux Belgium RL $160 \mathrm{~W}, 225$ -235 Volts $)$. After 10 minutes, $0.1 \mathrm{~mL}(0.66 \mathrm{mmol})$ of DBU was added and the reaction mixture was allowed to warm to room temperature. The solvent was removed in vacuo and the residue was purified by preparative thin layer chromatography $(30 \%$ acetone/hexanes) to give $18 \mathrm{mg}(51 \%)$ of sandresolide B (3) as a white solid.

\begin{tabular}{cccc}
\hline${ }^{1} \mathrm{H}$ NMR isolation & ${ }^{1} \mathrm{H}$ NMR current & ${ }^{13} \mathrm{C}$ NMR isolation & ${ }^{13} \mathrm{C}$ NMR current \\
\hline $5.05 \mathrm{br} \mathrm{d},(10.0 \mathrm{~Hz})$ & $5.05 \mathrm{brd}(9.9 \mathrm{~Hz})$ & 170.8 & 170.7 \\
$3.02 \mathrm{ddd}(10.0,8.5,3.9$ & $3.01 \mathrm{ddd}(9.9,8.2$, & 162.0 & 161.9 \\
$\mathrm{~Hz})$ & $3.6 \mathrm{~Hz})$ & & \\
$2.53, \mathrm{~m}$ & $2.55, \mathrm{~m}$ & 134.8 & 134.7 \\
$2.18, \mathrm{~m}$ & $2.19, \mathrm{~m}$ & 132.4 & 132.4 \\
$2.08, \mathrm{~m}$ & $2.08, \mathrm{~m}$ & 124.3 & 124.3 \\
$2.00, \mathrm{~m}$ & $2.00, \mathrm{~m}$ & 108.2 & 108.2 \\
$2.00, \mathrm{~m} *$ & $1.27, \mathrm{~m}$ & 77.2 & 77.3 \\
$1.92, \mathrm{~m}$ & $1.92, \mathrm{~m}$ & 46.0 & 46.1 \\
$1.77, \mathrm{~d}, 1.2 \mathrm{~Hz}$ & $1.77, \mathrm{~d},(1.9 \mathrm{~Hz})$ & 43.9 & 43.9 \\
$1.72, \mathrm{~d}, 1.1 \mathrm{~Hz}$ & $1.73, \mathrm{~d},(1.4 \mathrm{~Hz})$ & 43.8 & 43.8 \\
$1.57, \mathrm{~m}$ & $1.57(\mathrm{~m})$ & 33.3 & 33.2 \\
$1.24, \mathrm{~m}$ & $1.28(\mathrm{~m})$ & 31.7 & 31.7 \\
$1.24, \mathrm{~d},(6.5 \mathrm{~Hz})$ & $1.24(\mathrm{~d}, 7.0 \mathrm{~Hz})$ & 28.2 & 28.2 \\
$1.18, \mathrm{~m}$ & $1.18(\mathrm{~m})$ & 27.5 & 27.5 \\
$1.12, \mathrm{~s}$ & $1.11(\mathrm{~s})$ & 26.2 & 26.3 \\
$0.94, \mathrm{~d},(6.8 \mathrm{~Hz})$ & $0.95, \mathrm{~d},(6.9 \mathrm{~Hz})$ & 21.0 & 21.0 \\
& & 19.1 & 19.1 \\
& & 18.4 & 18.5 \\
\hline
\end{tabular}

* Error in isolation paper as discussed in manuscript, confirmed through correspondence with the isolationist. 


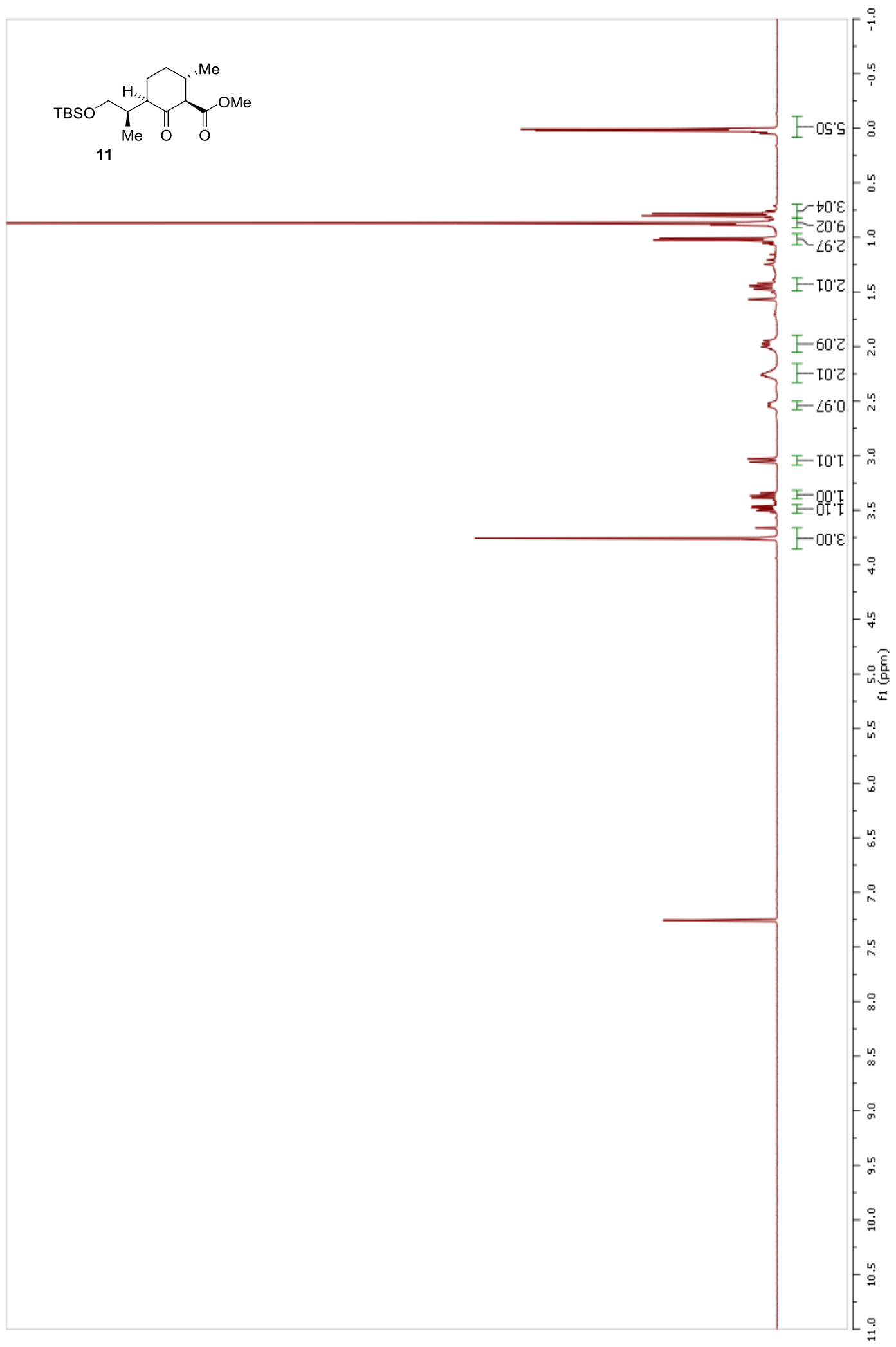



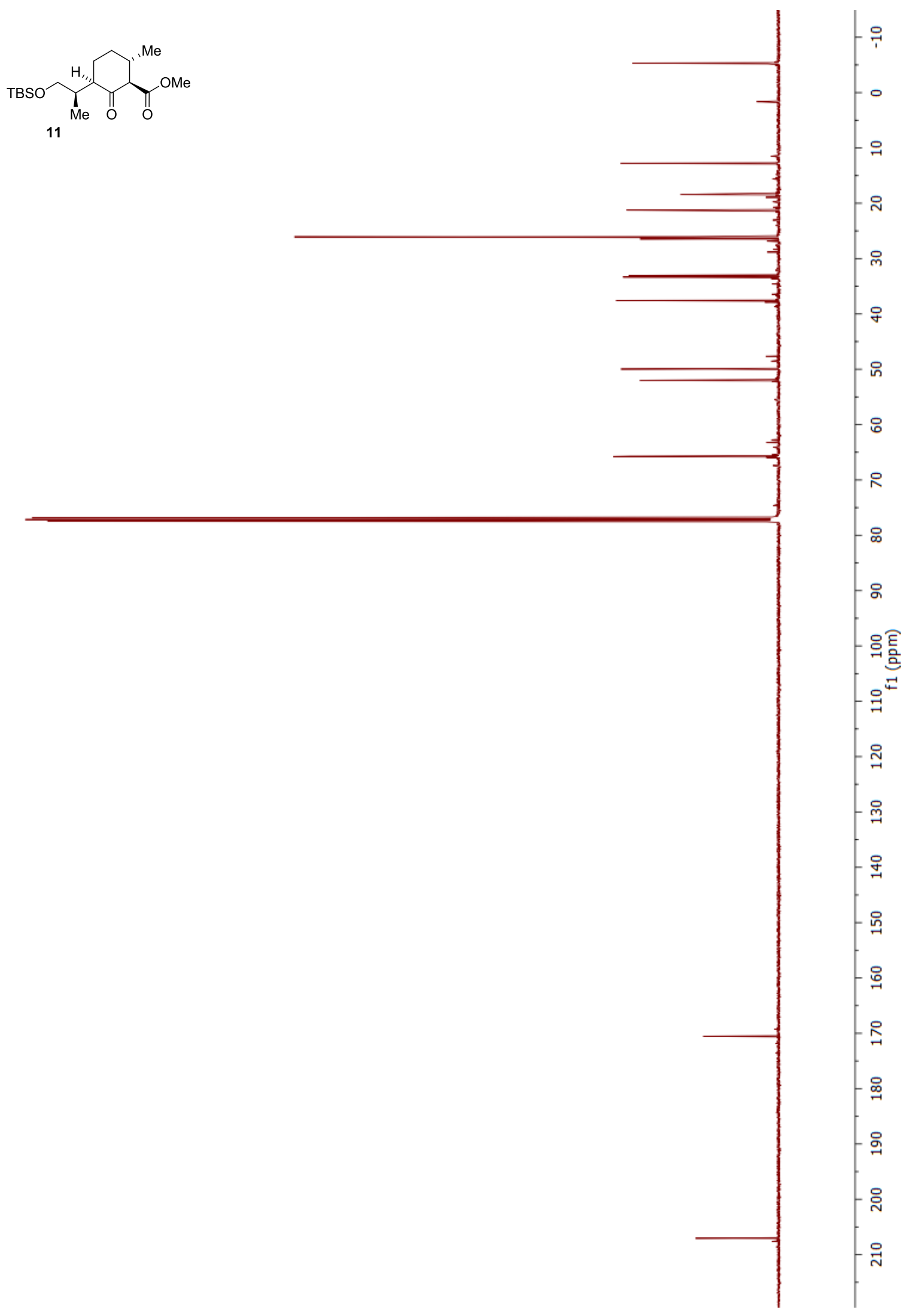


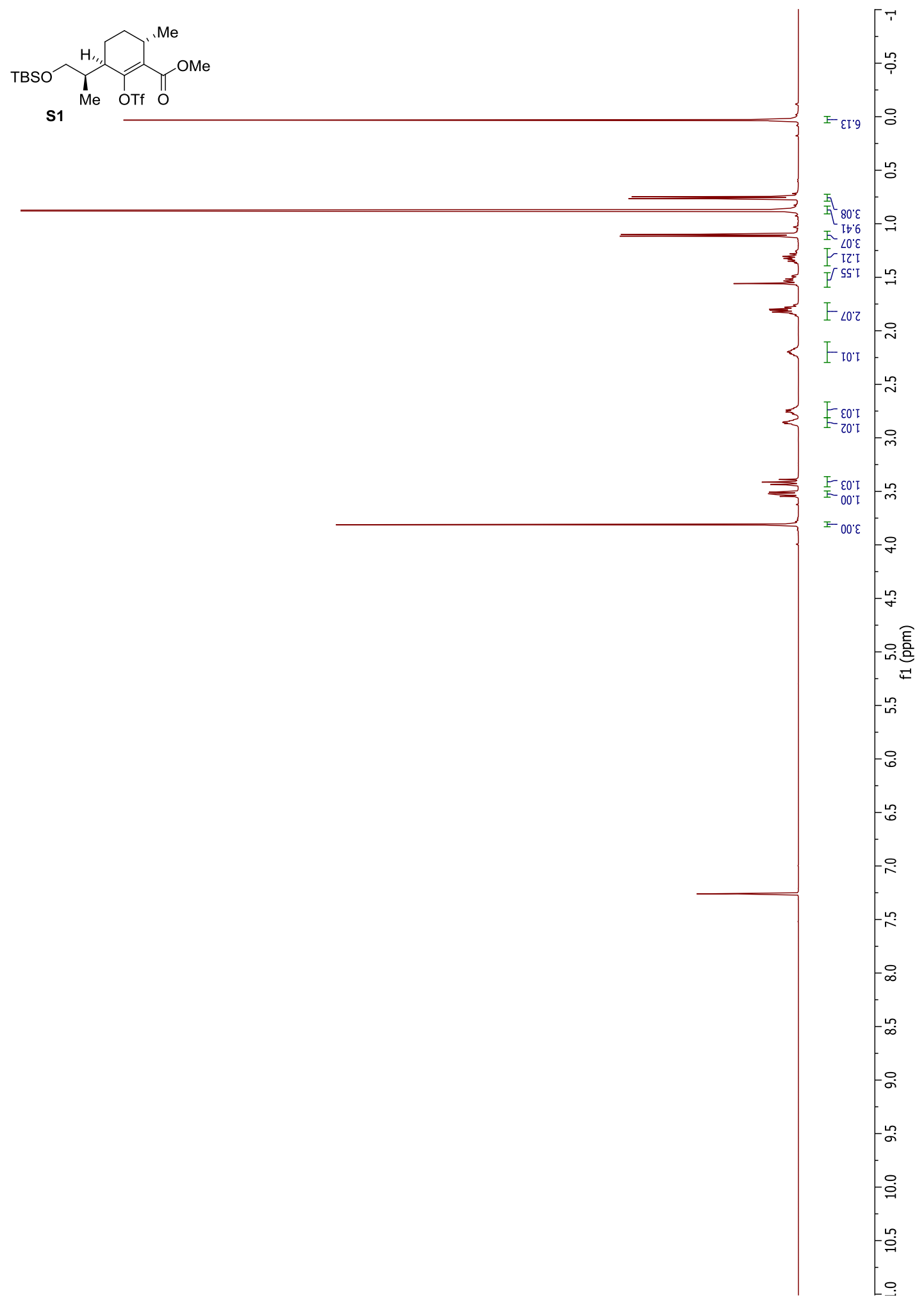



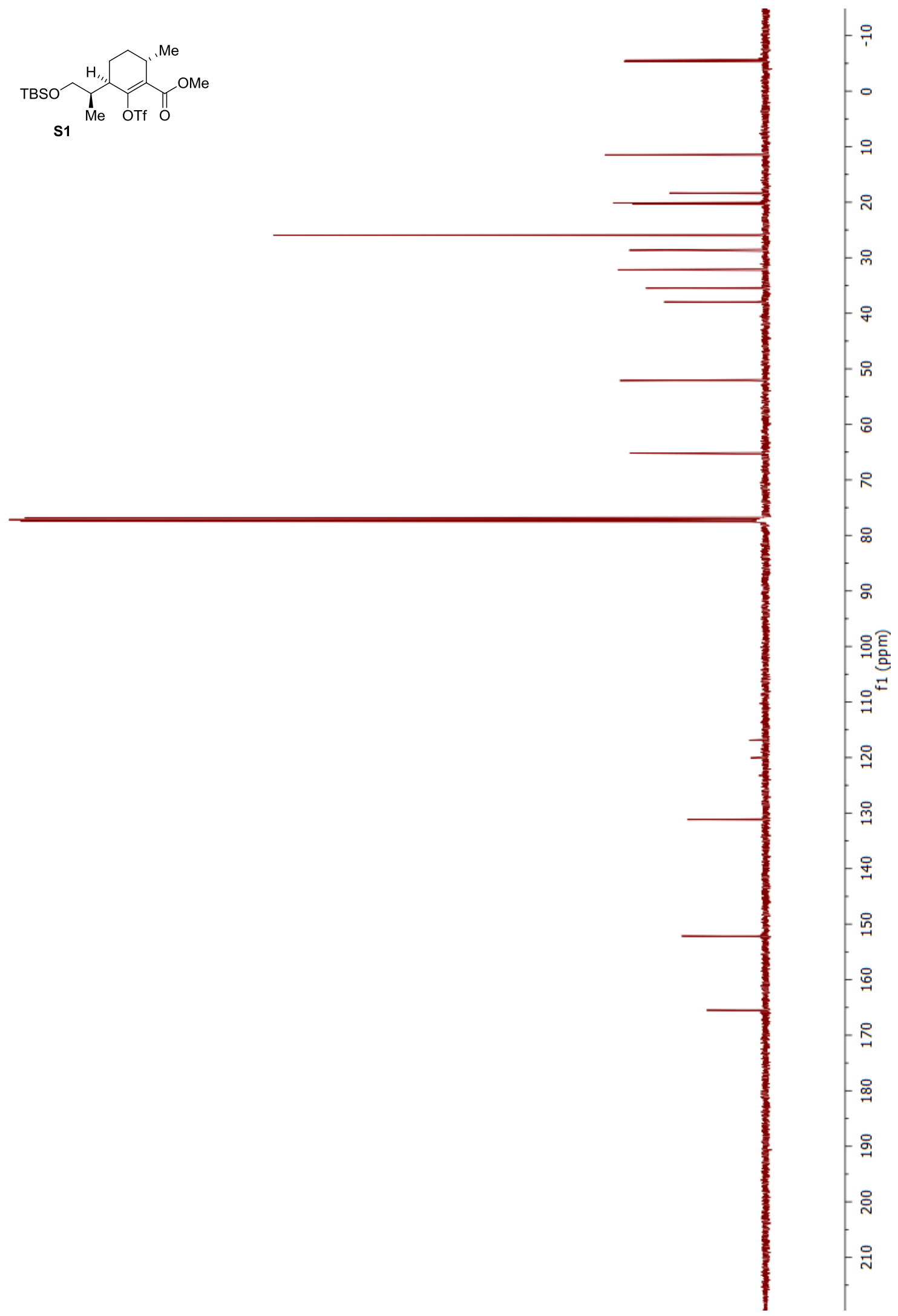


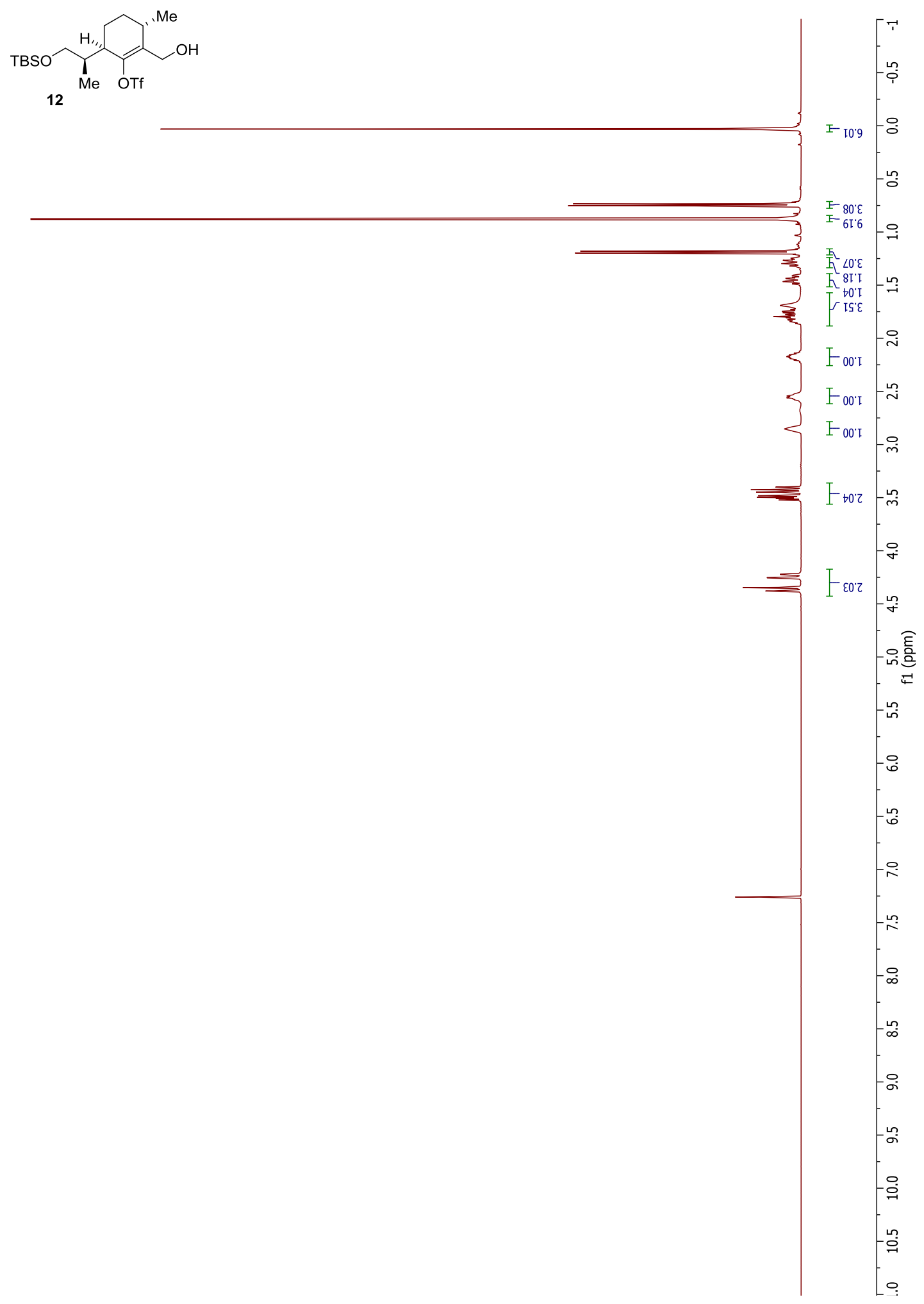



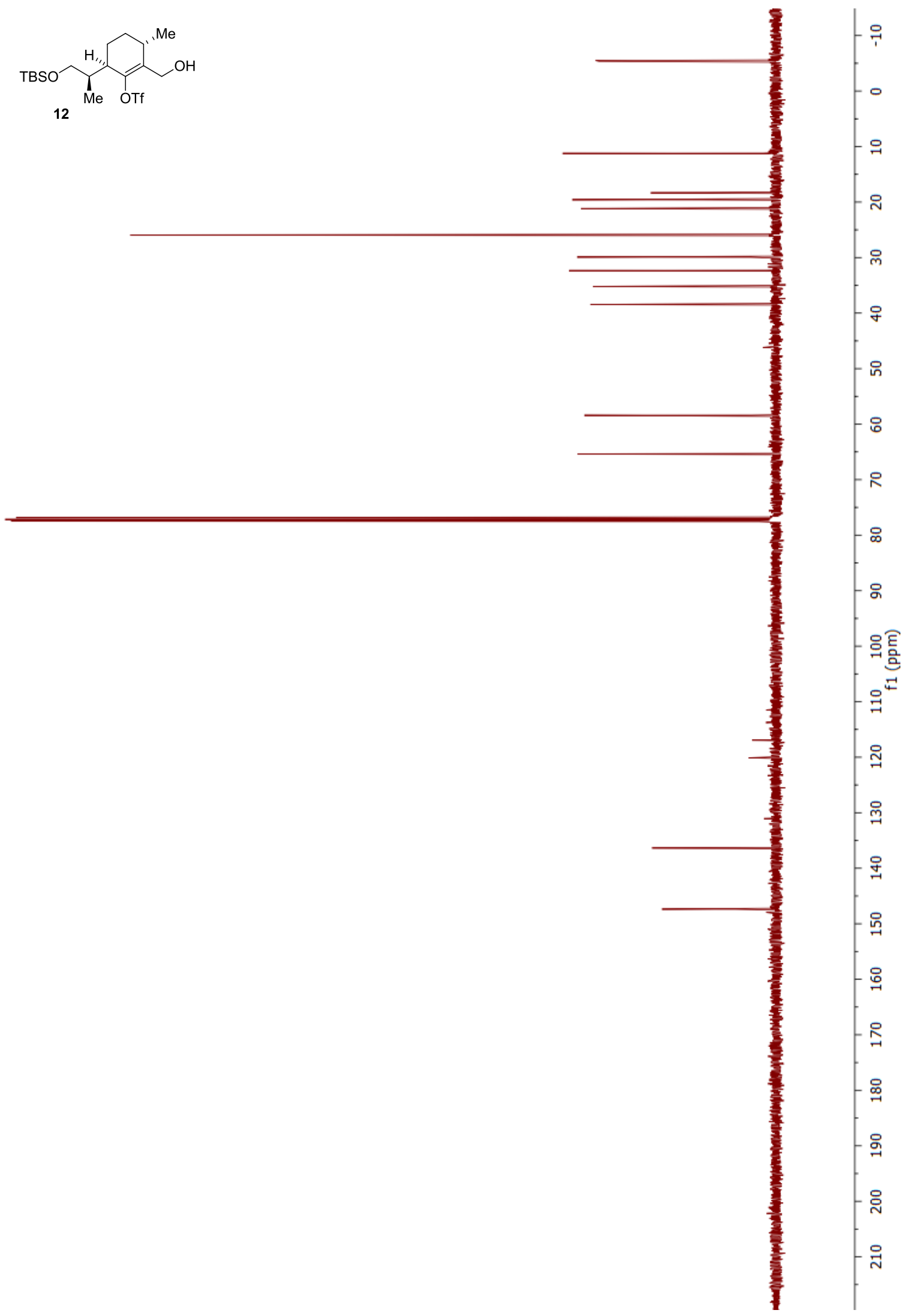


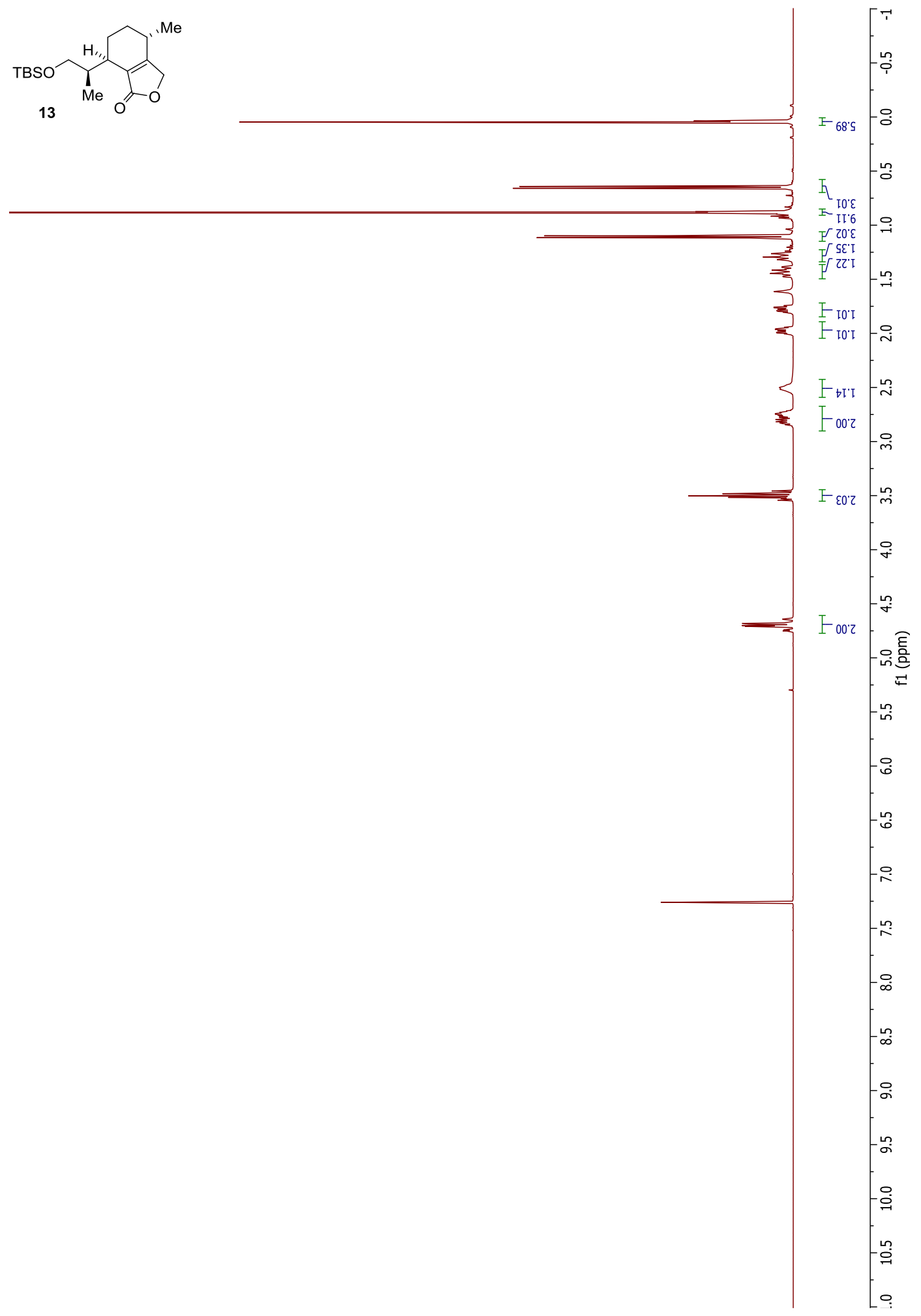



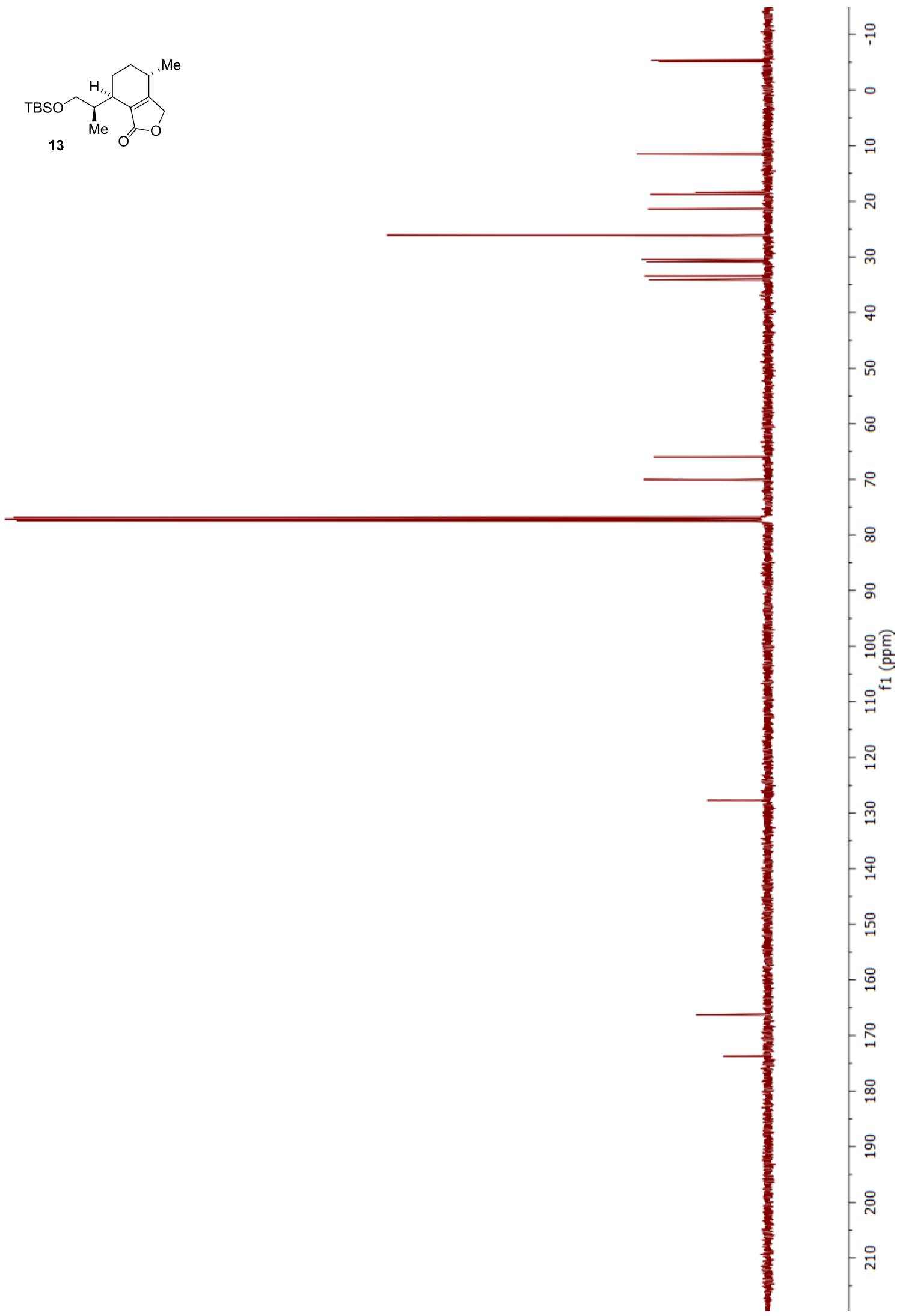


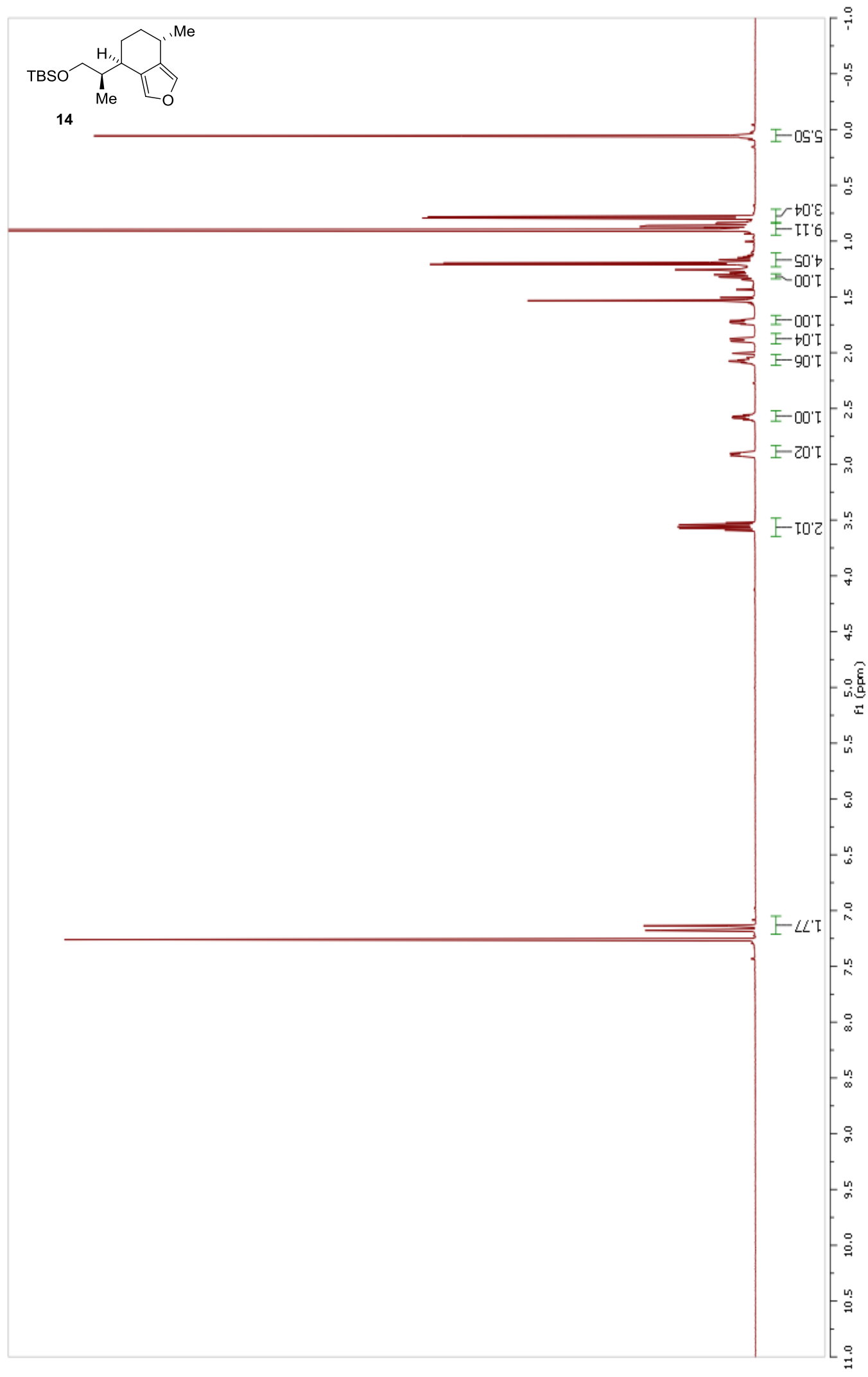




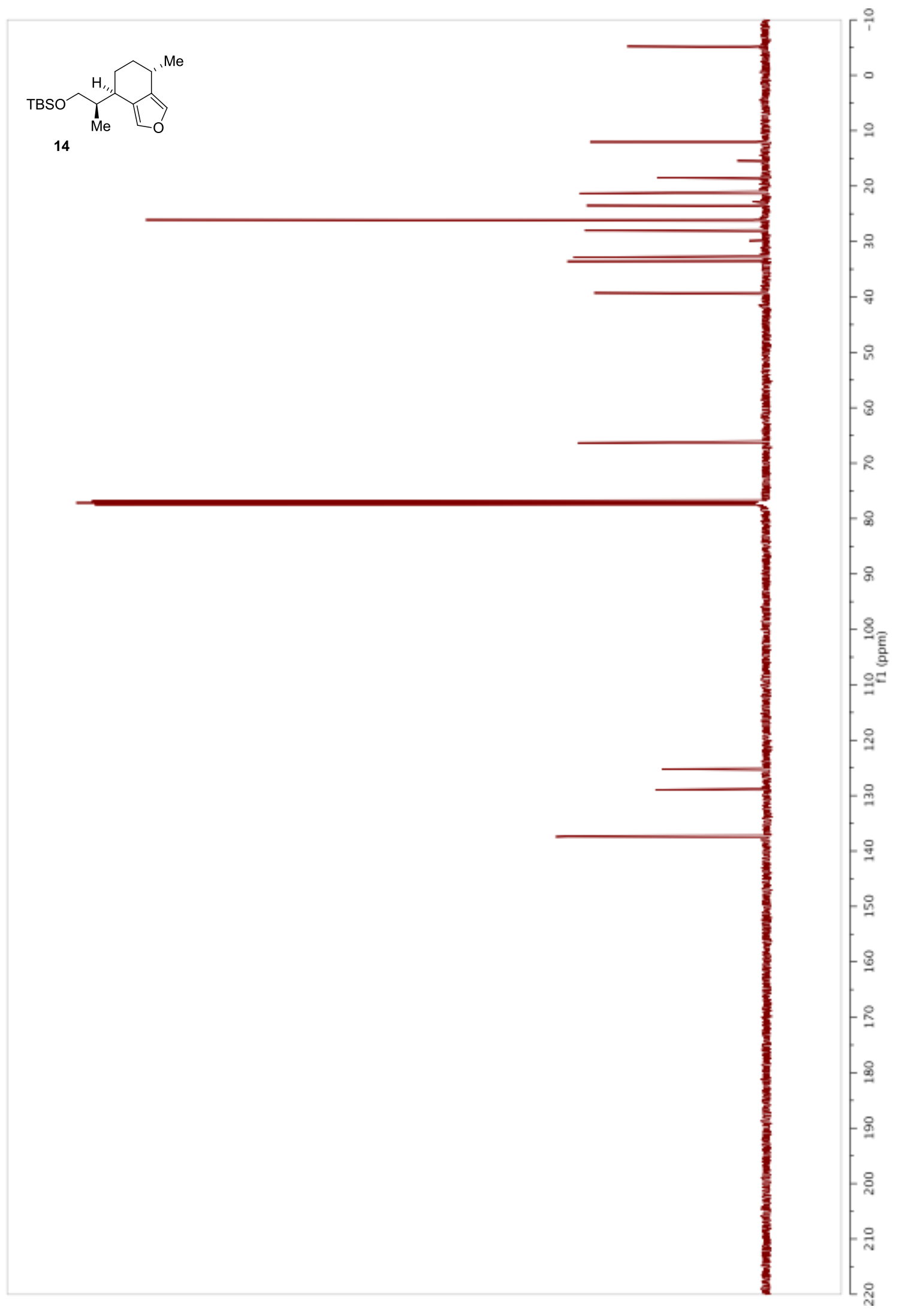




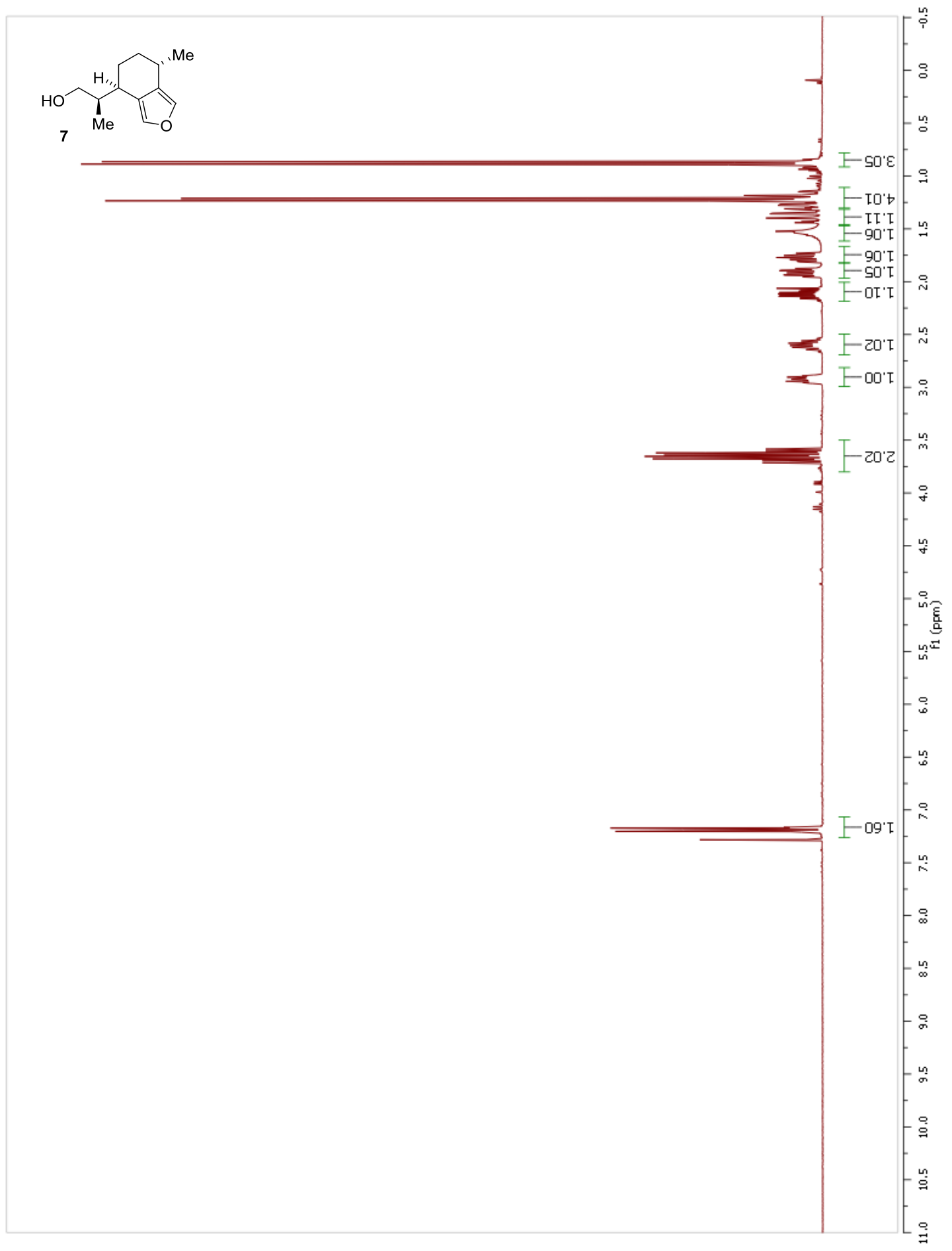




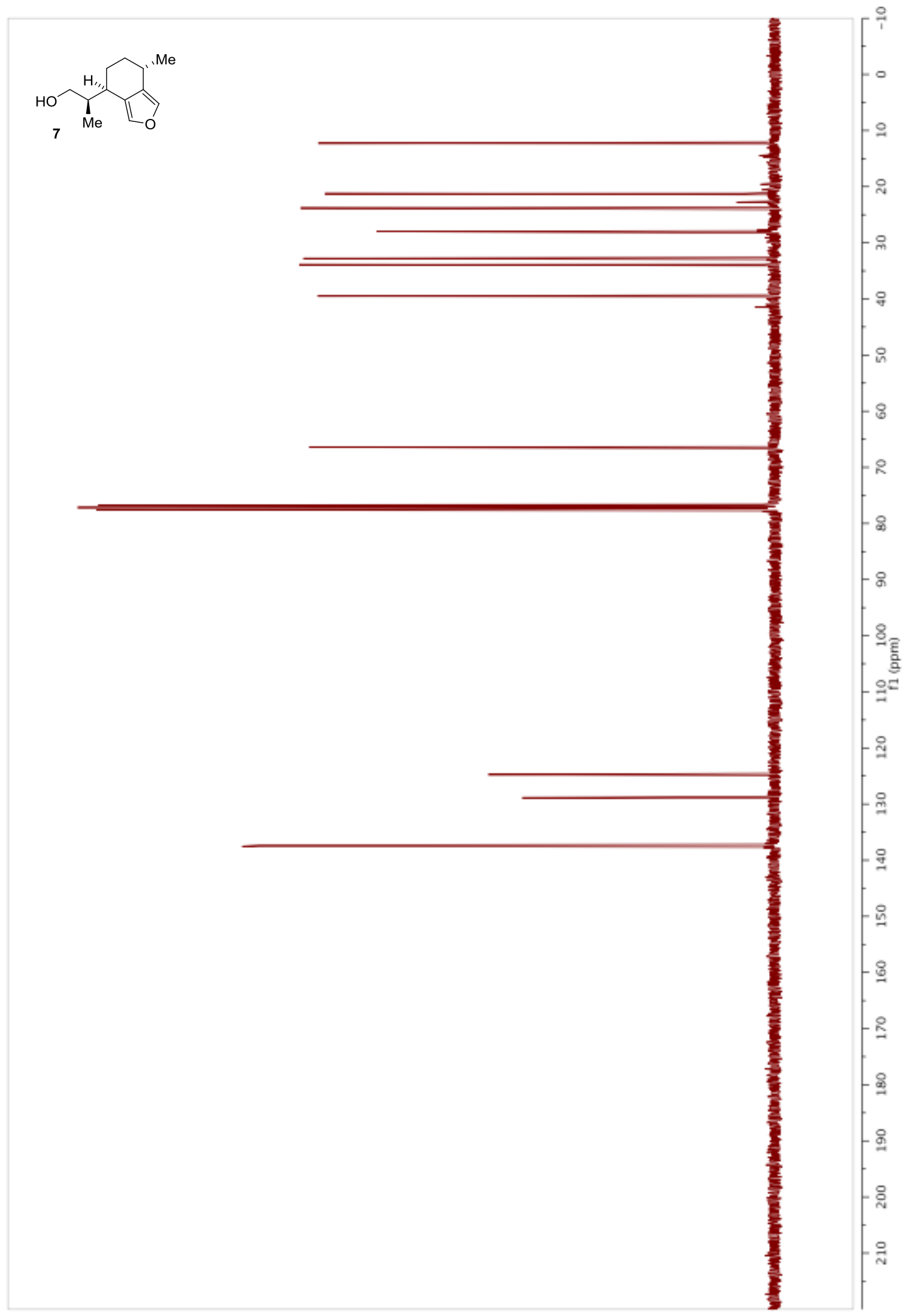




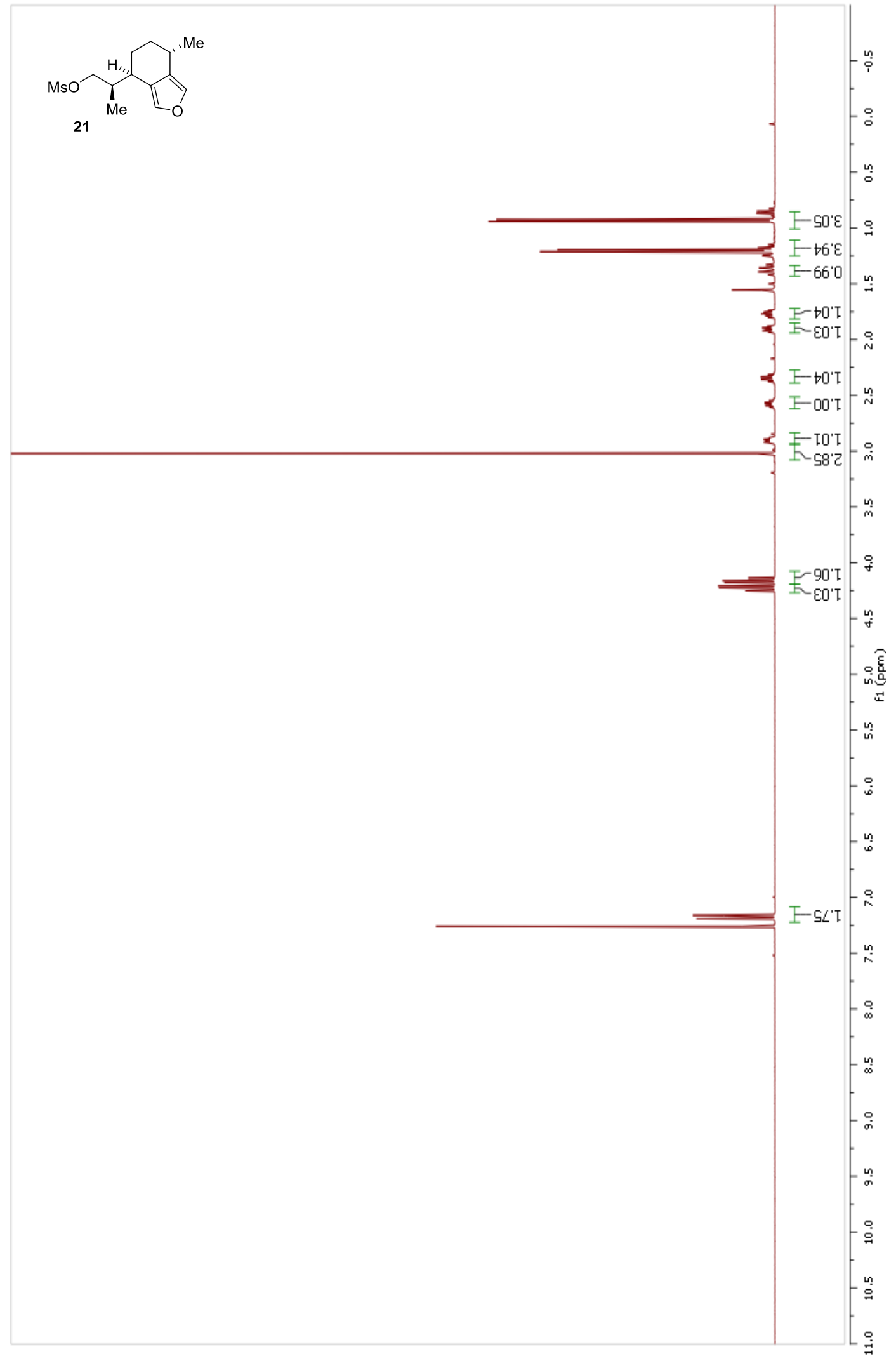




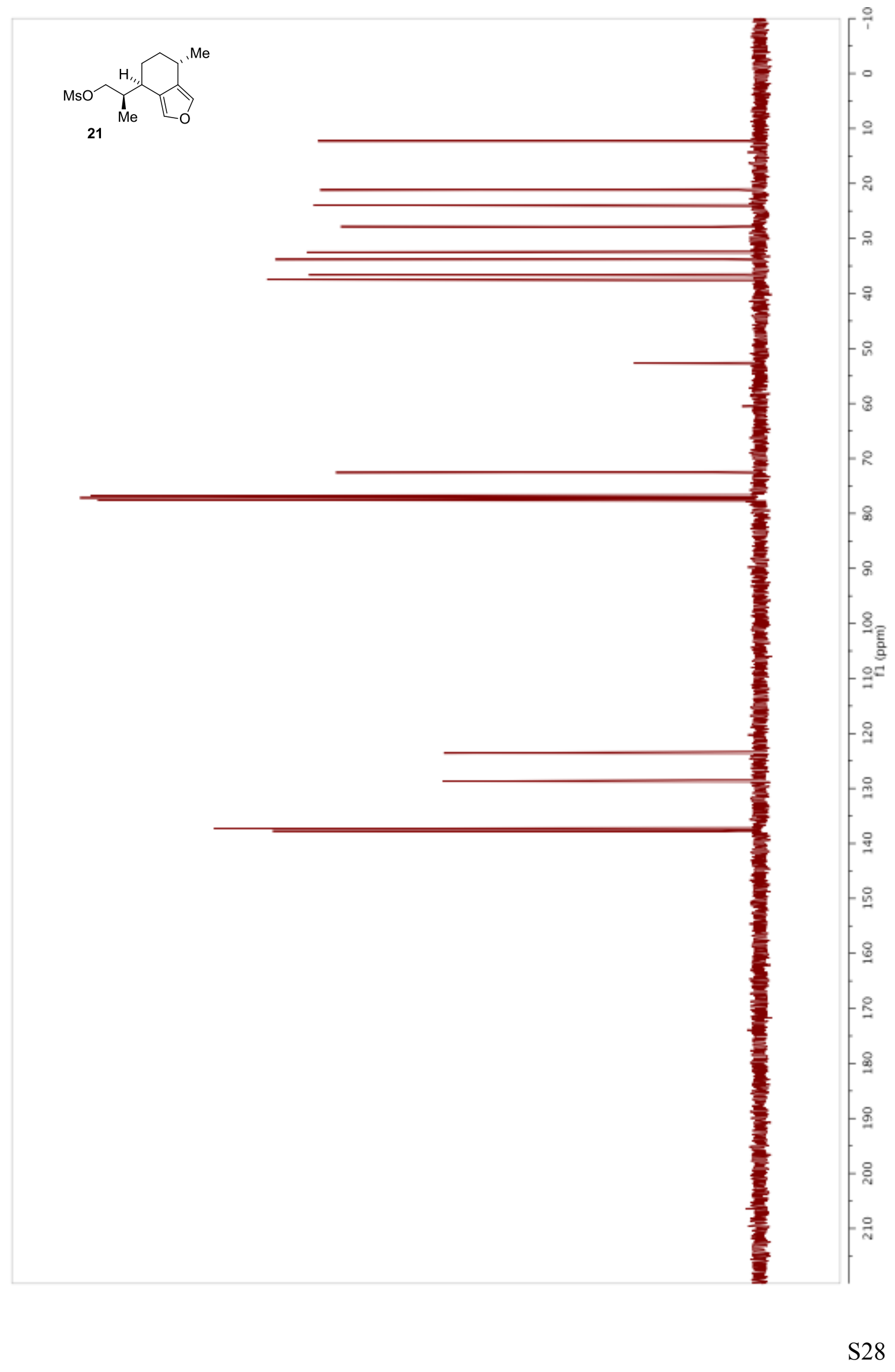




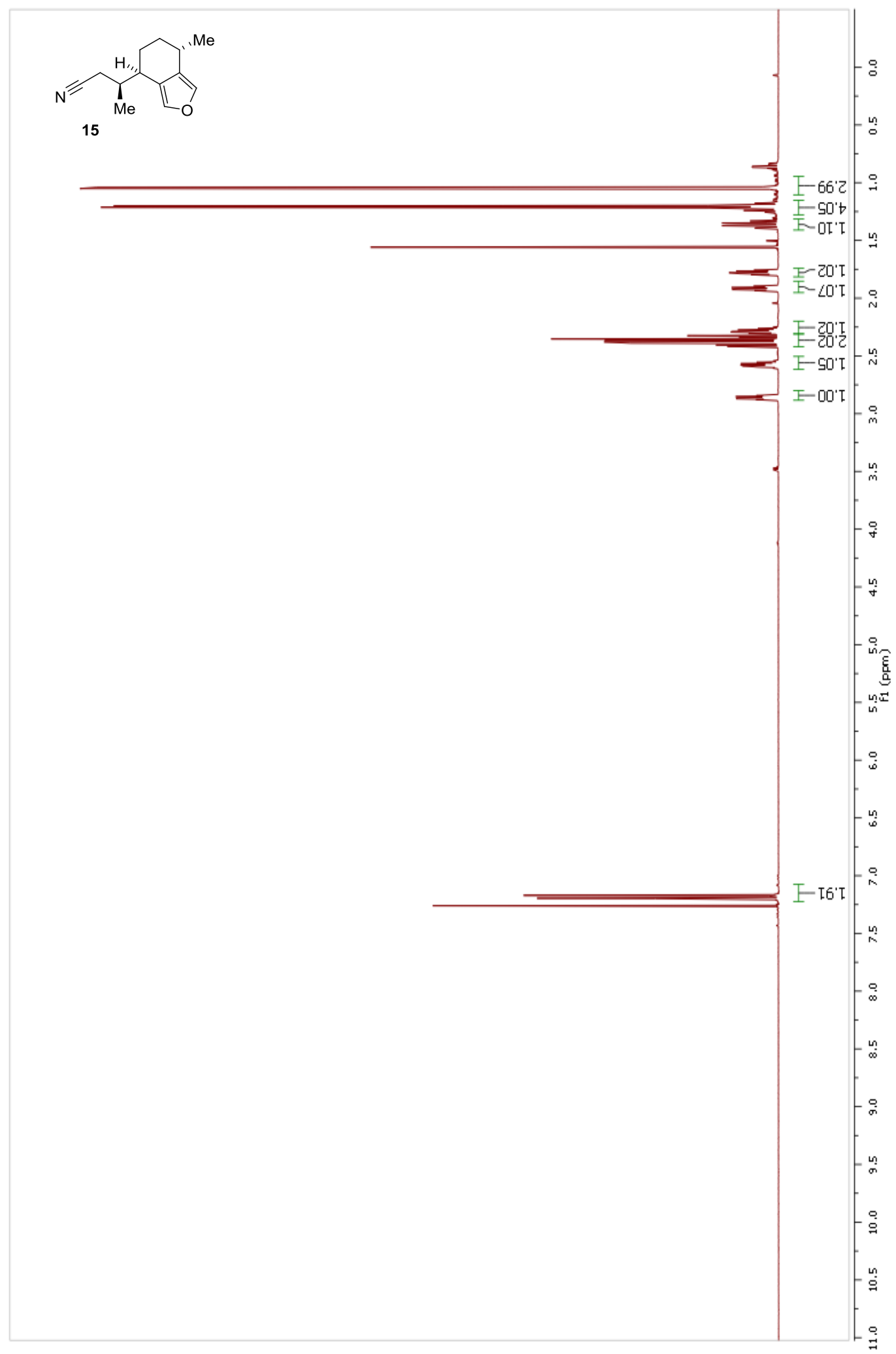




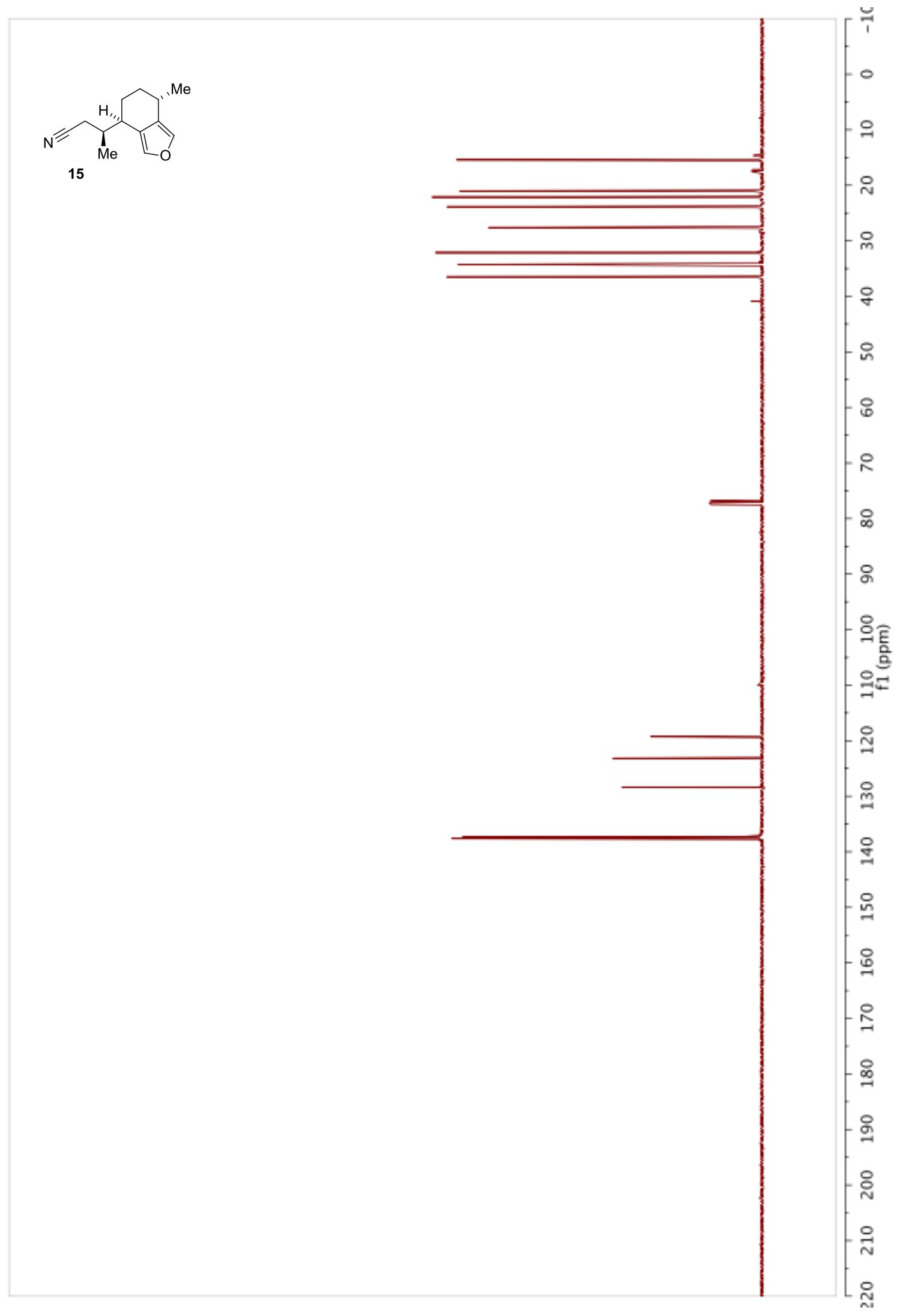




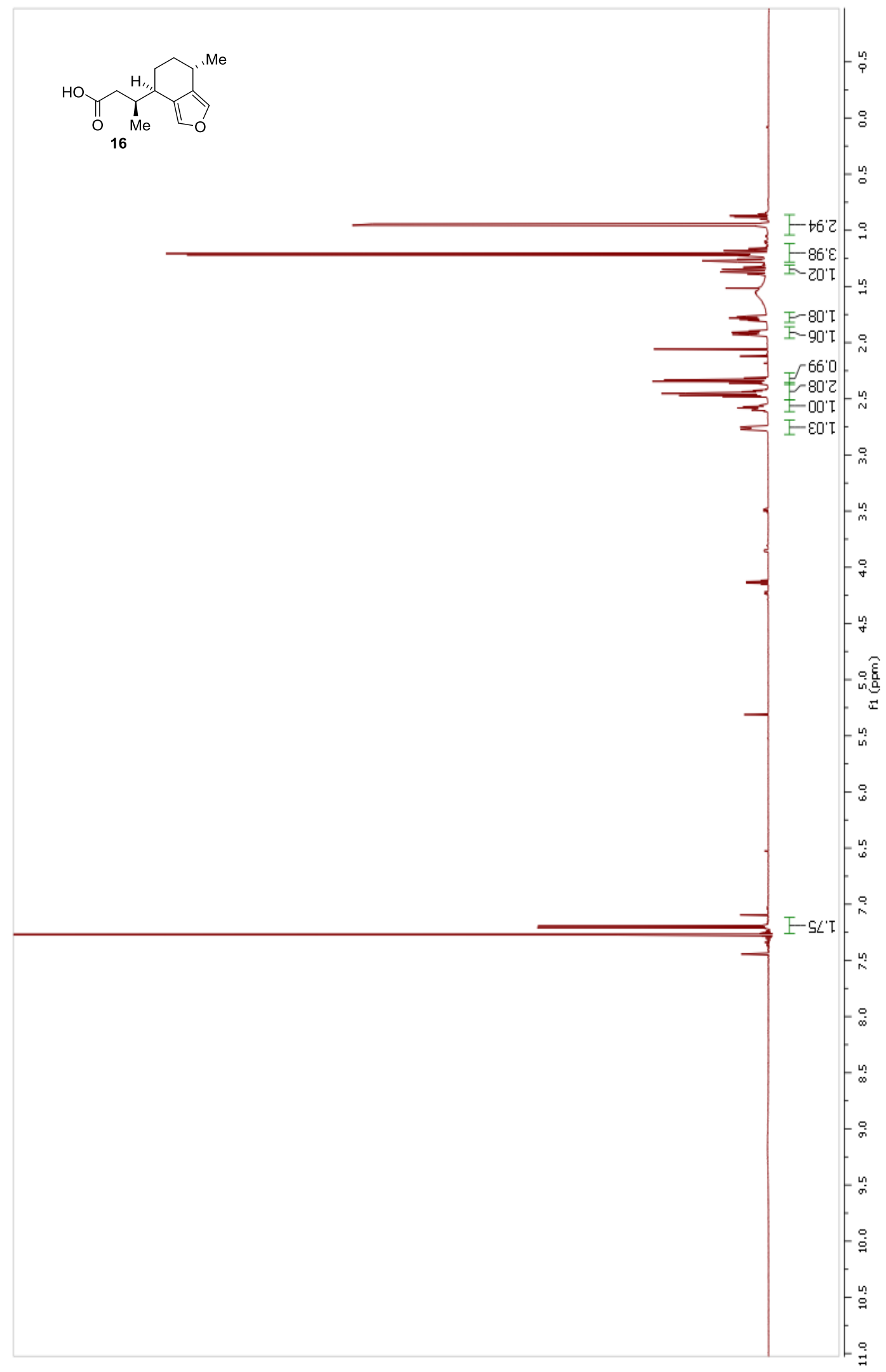



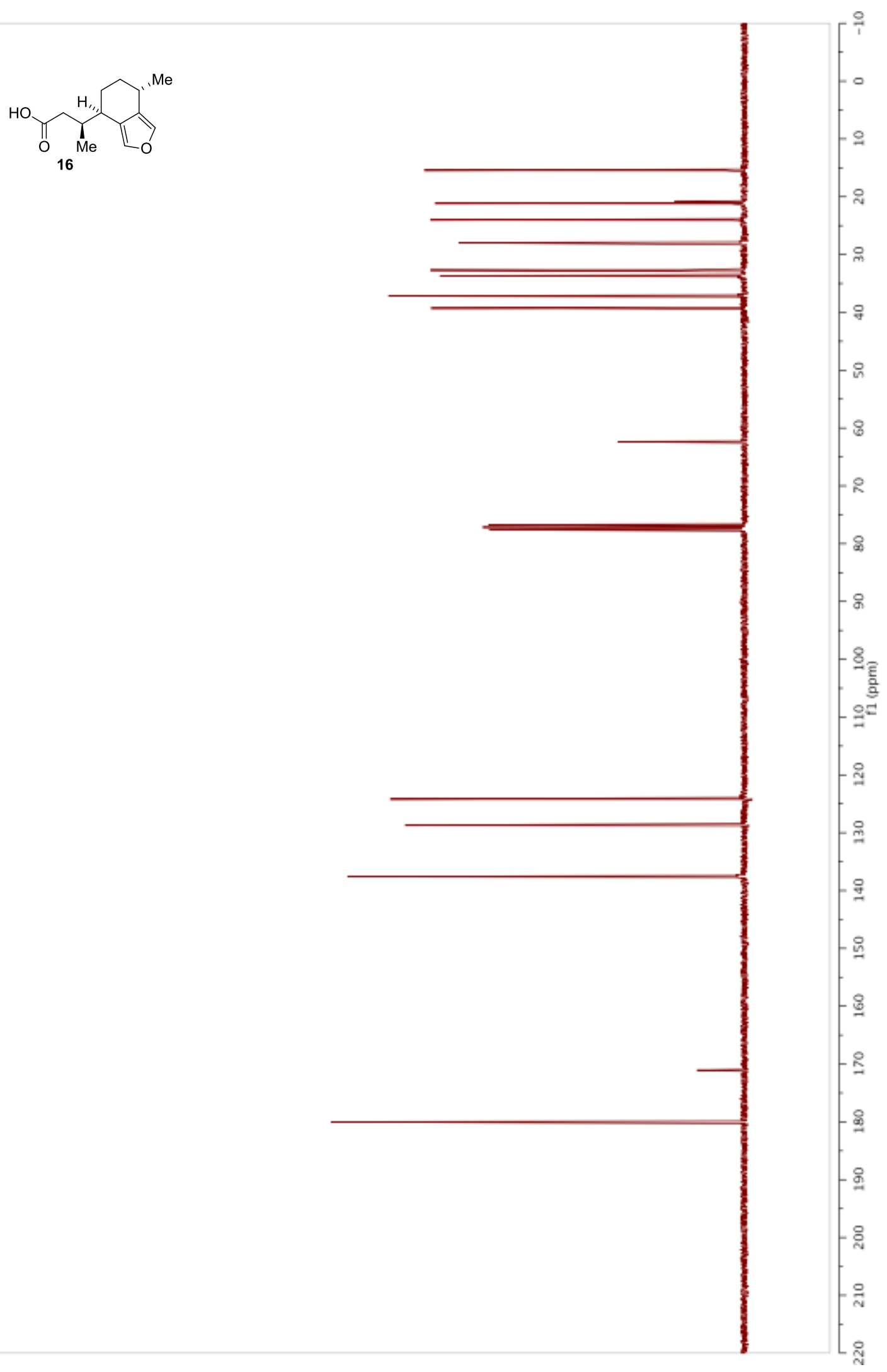

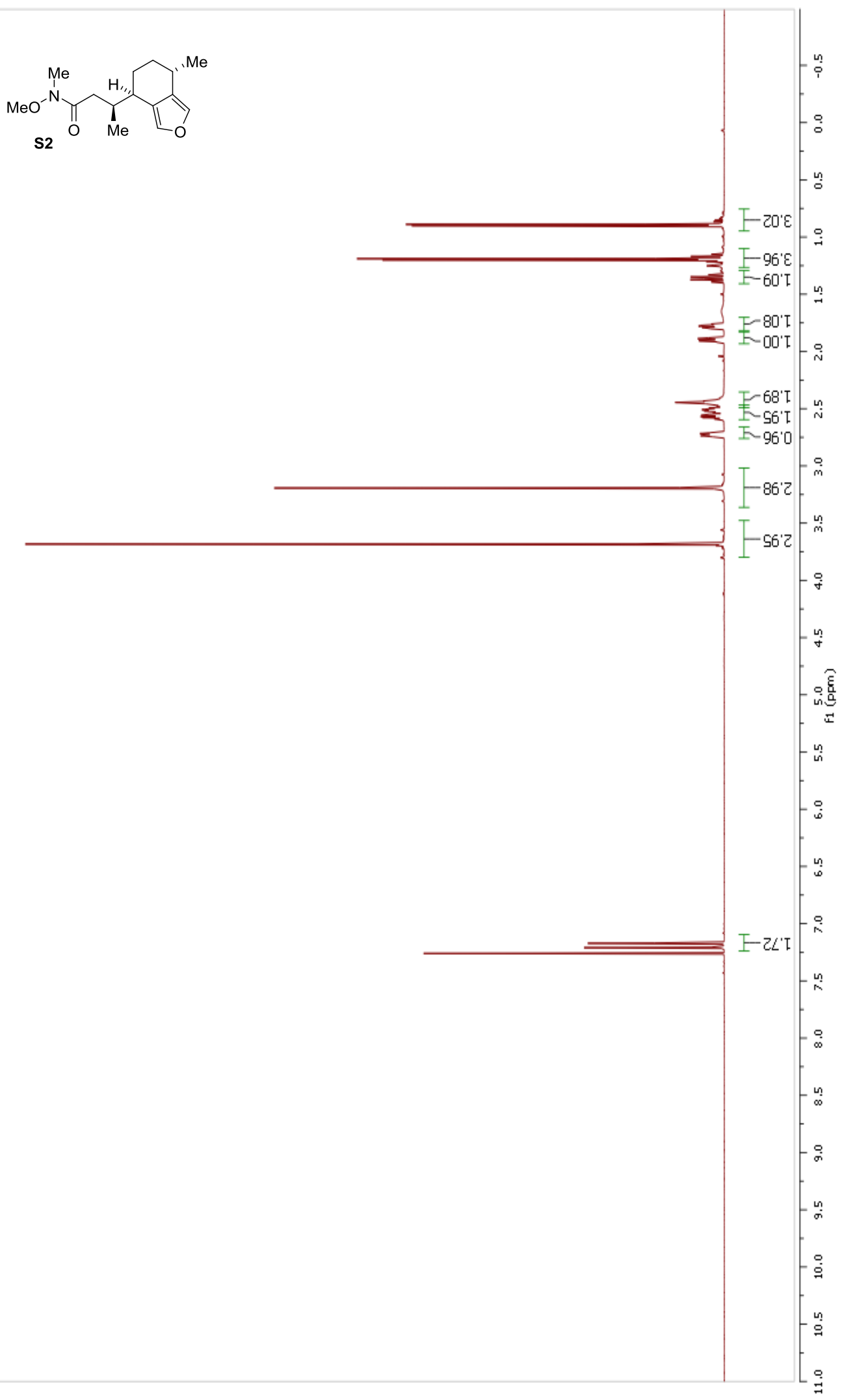


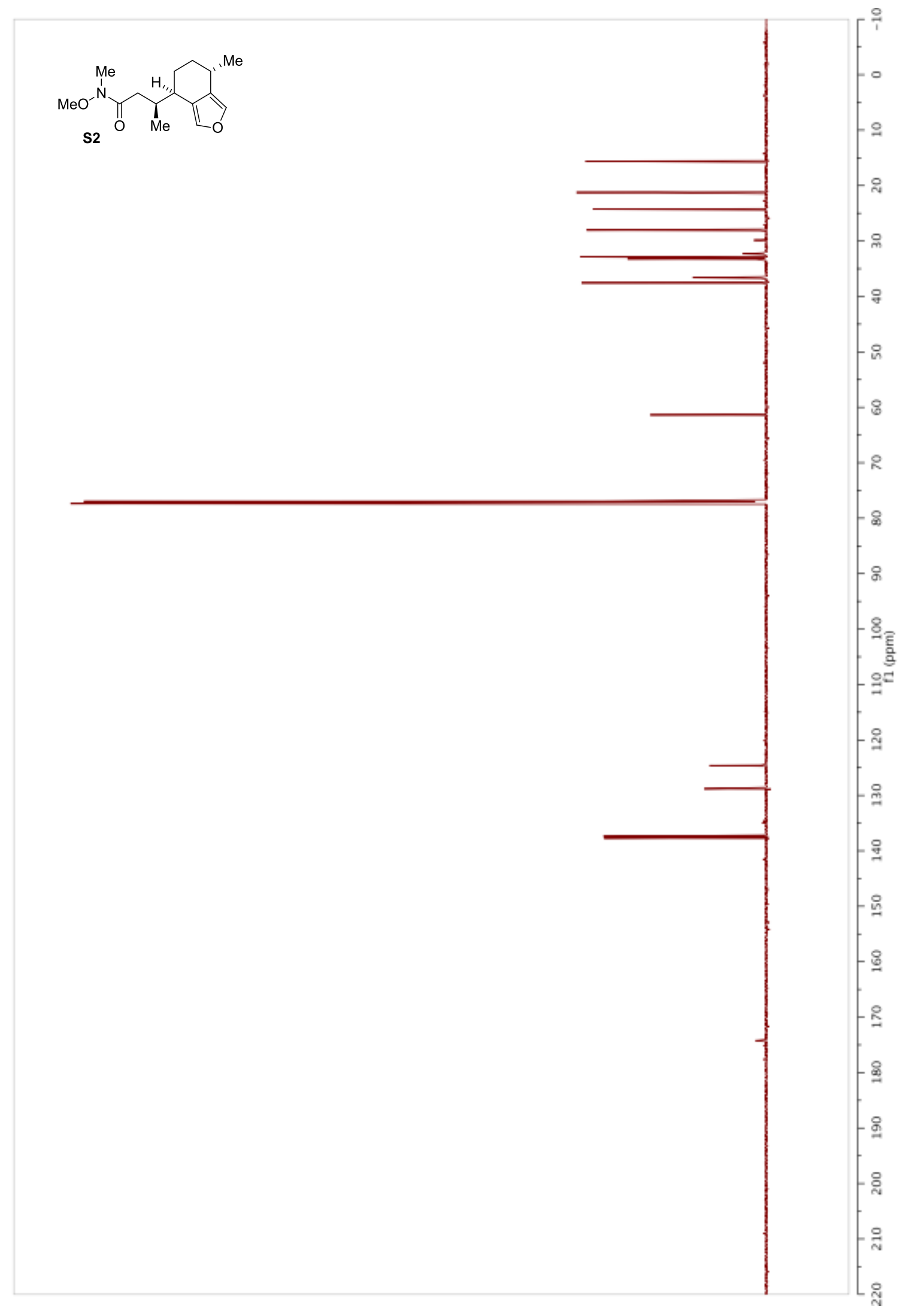




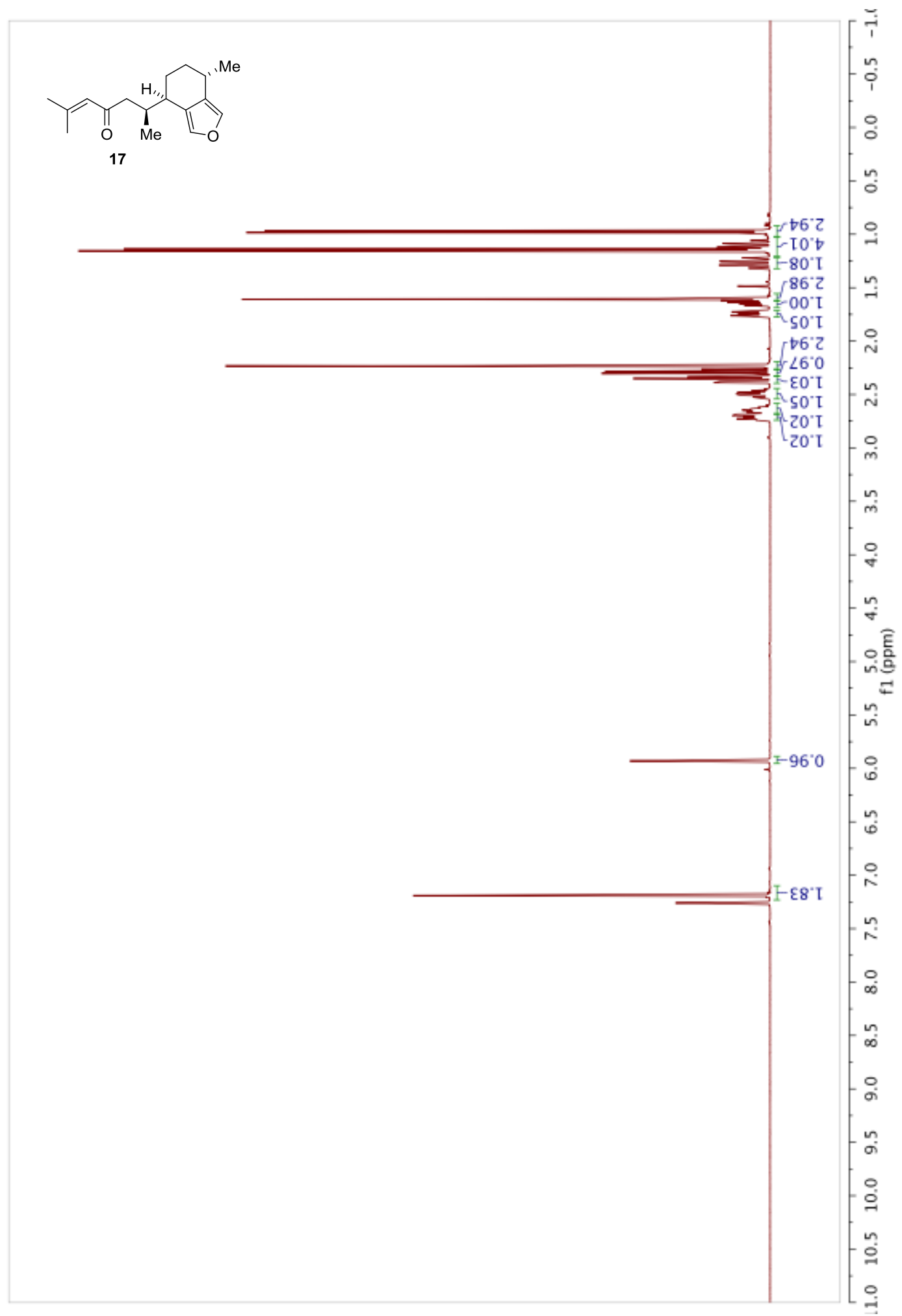




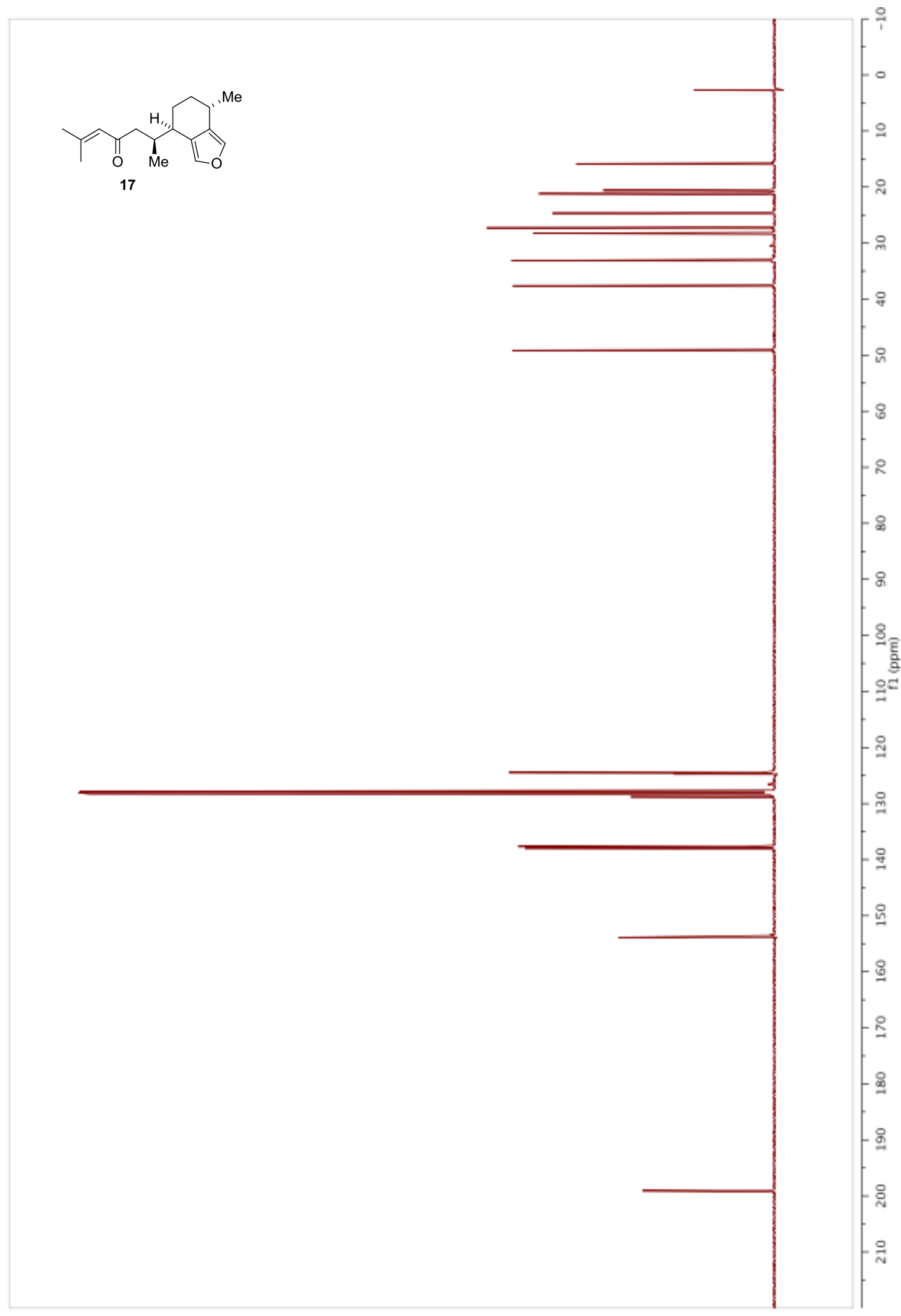




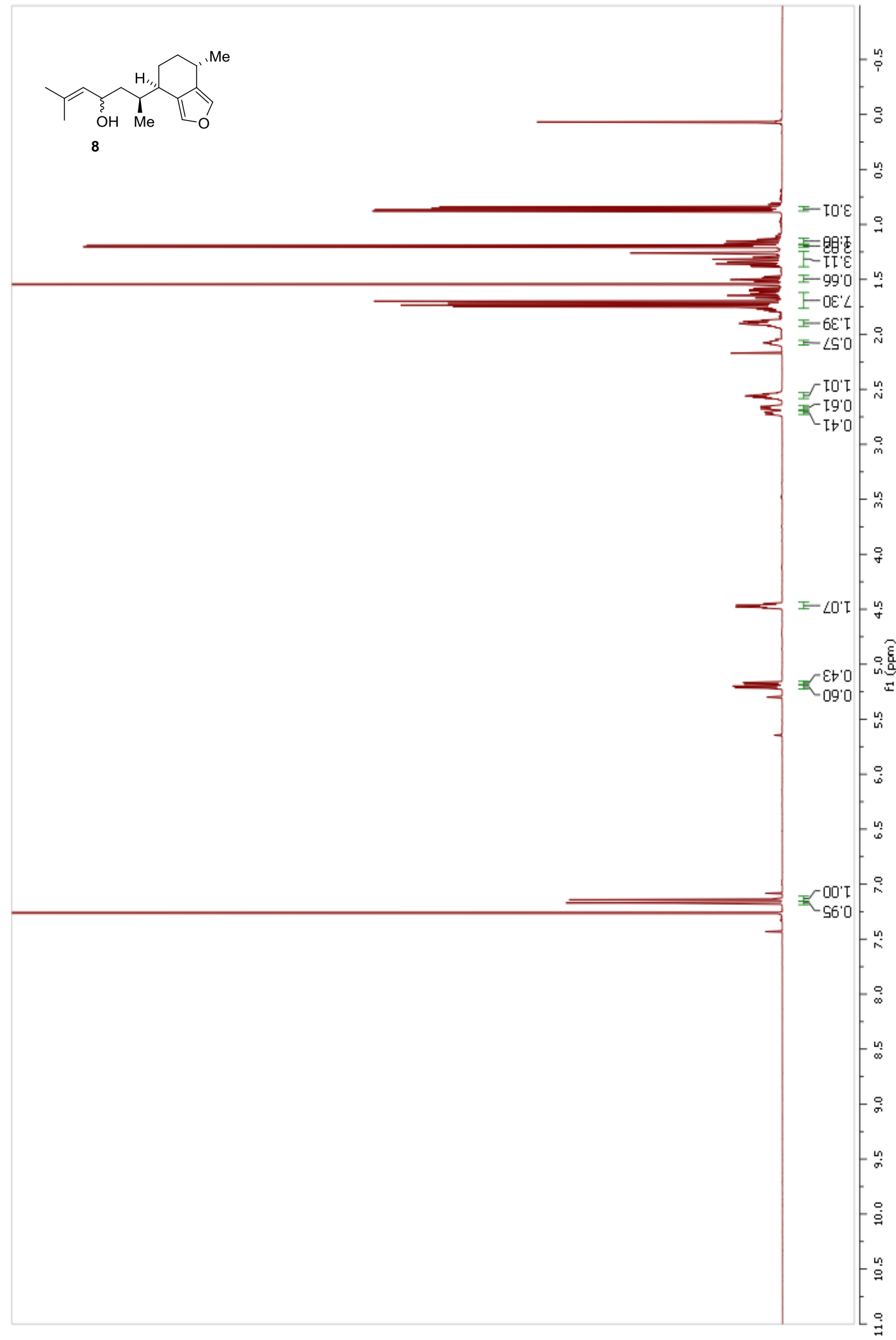




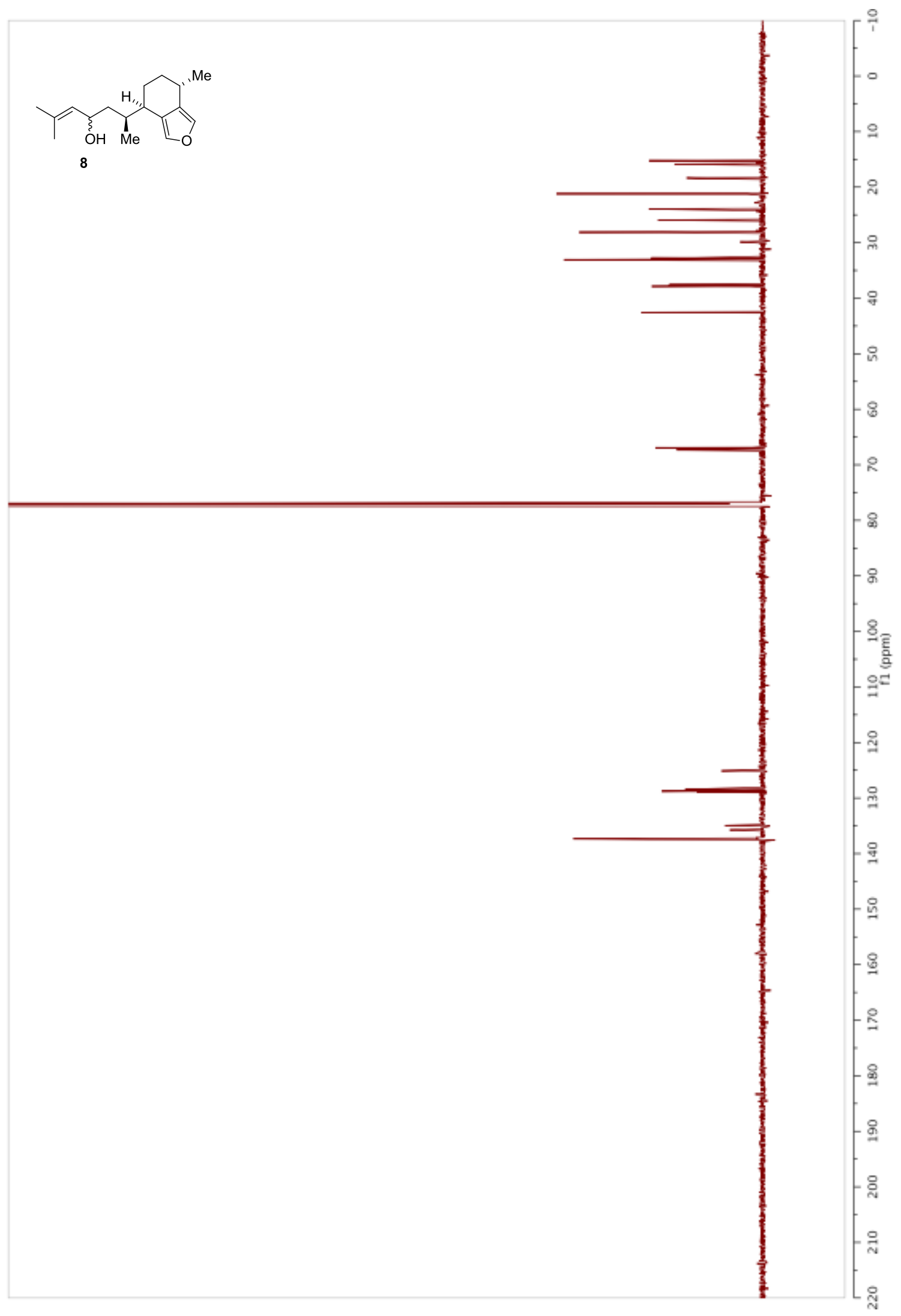




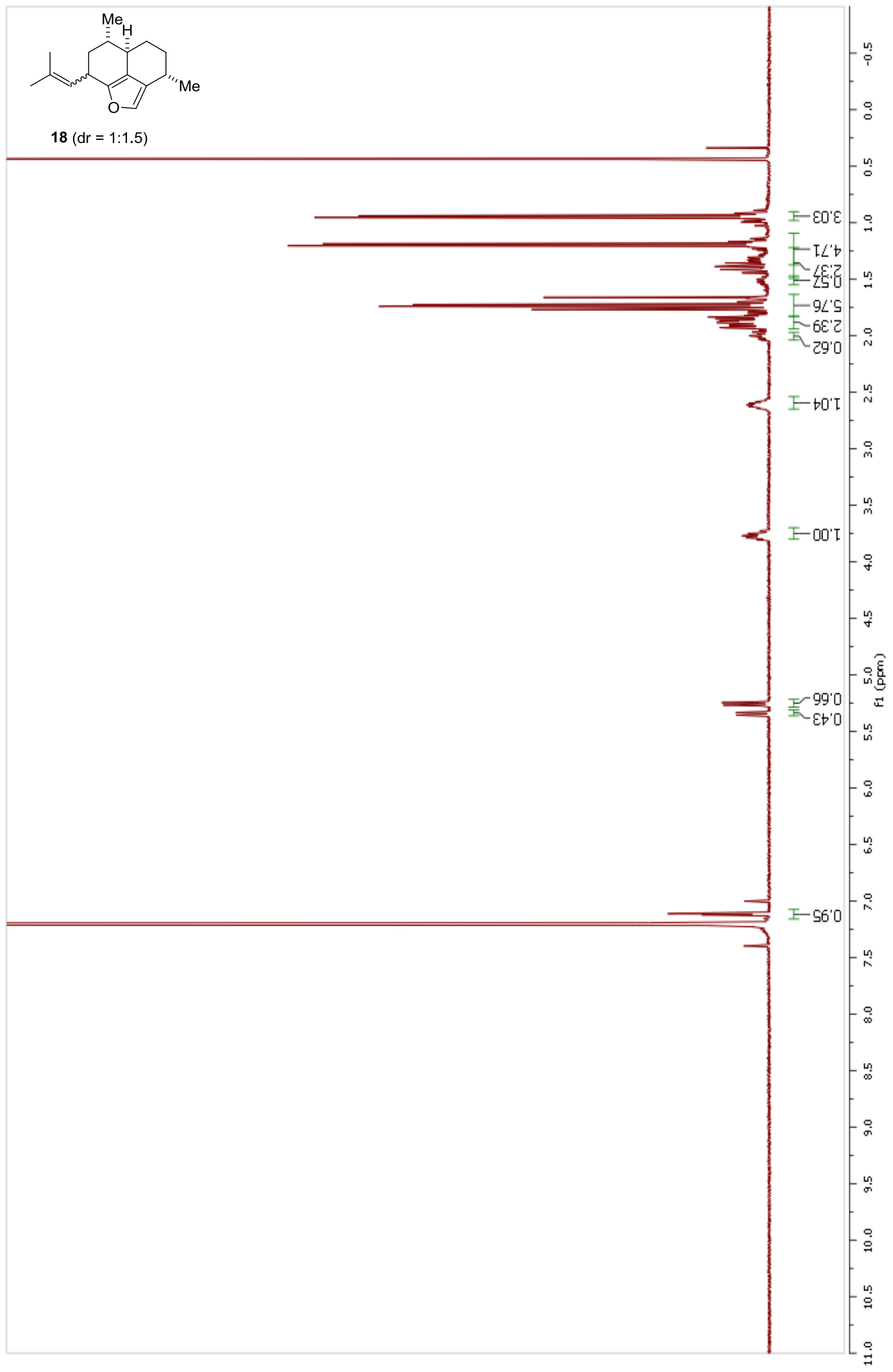




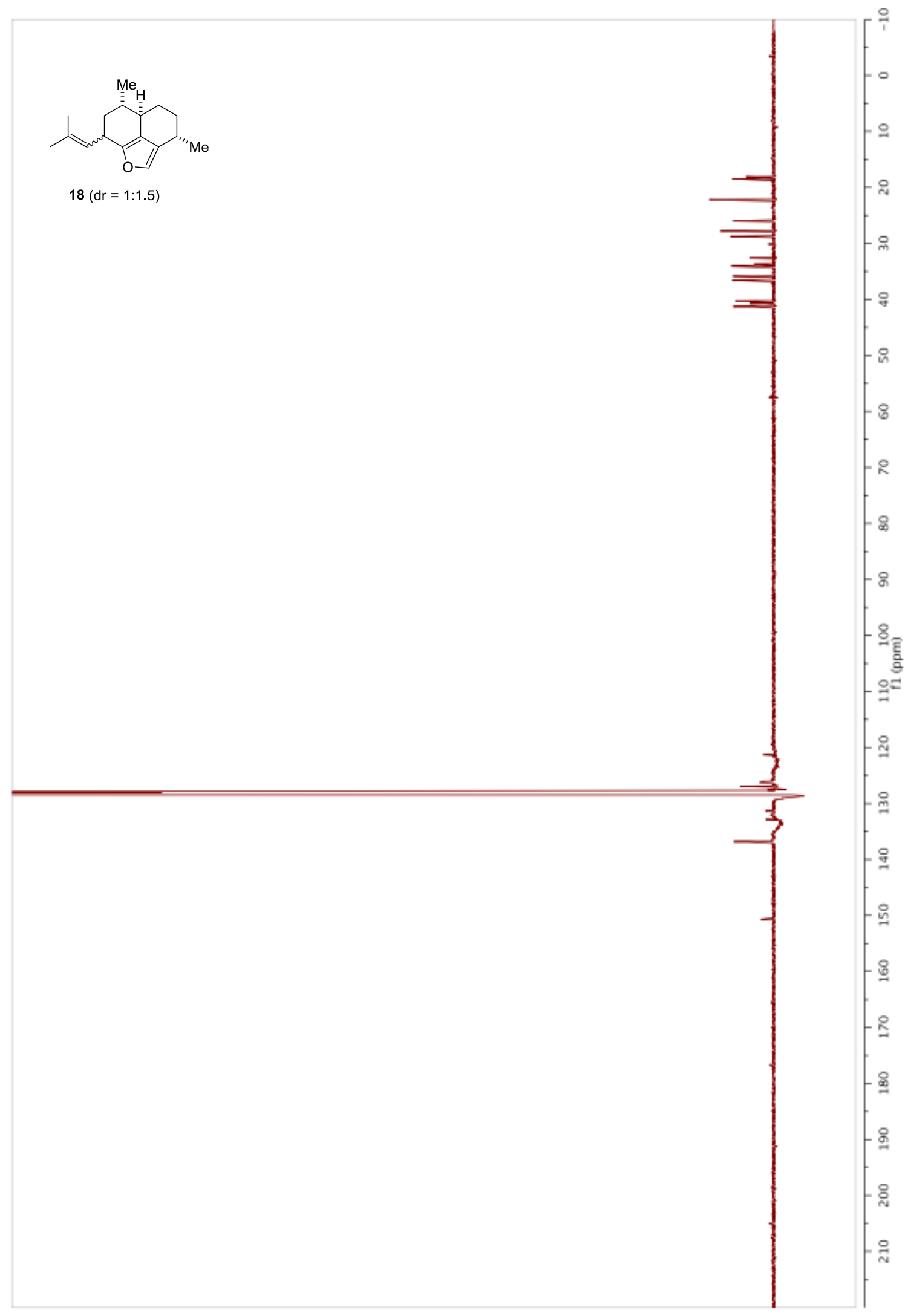




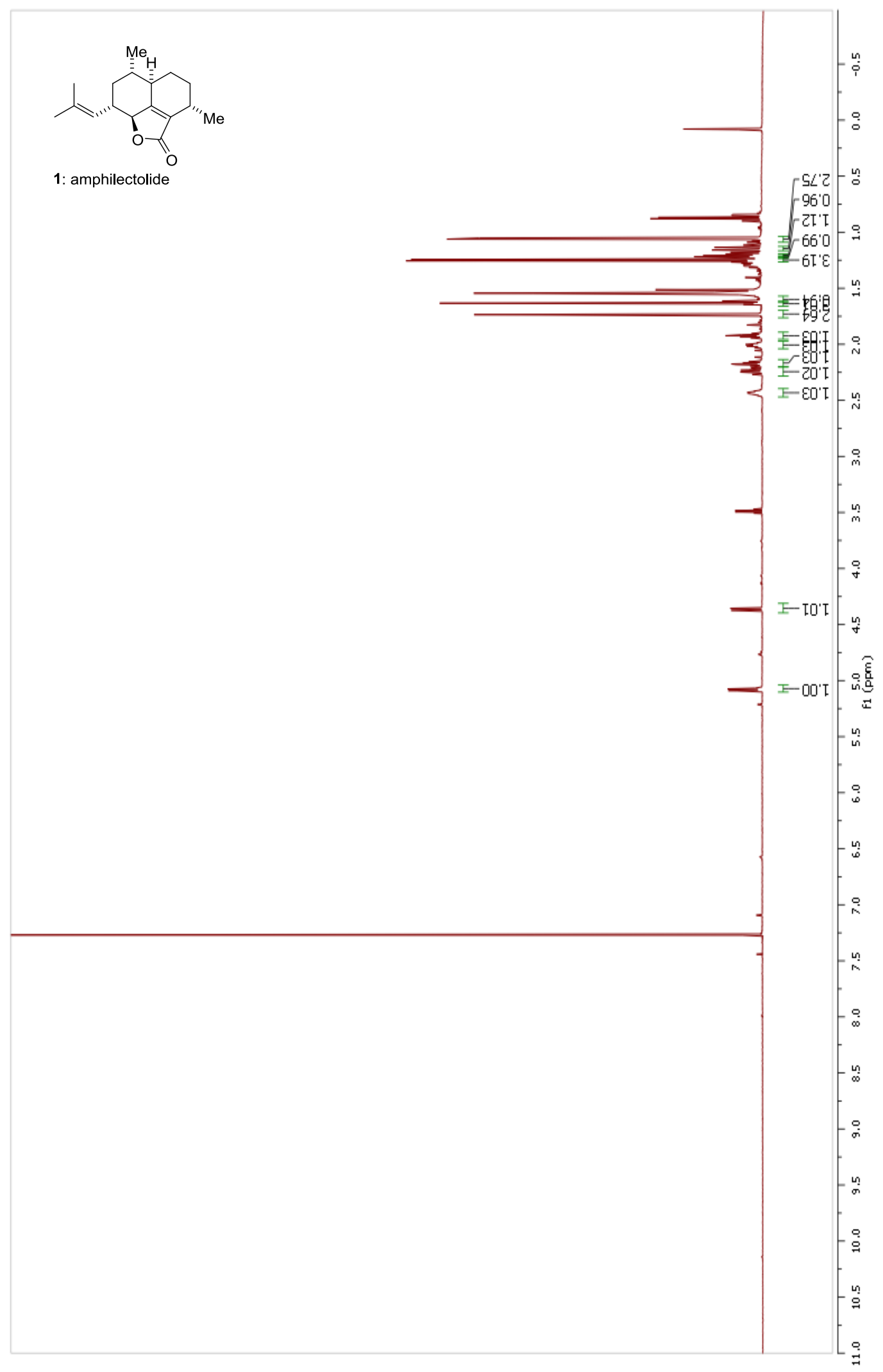




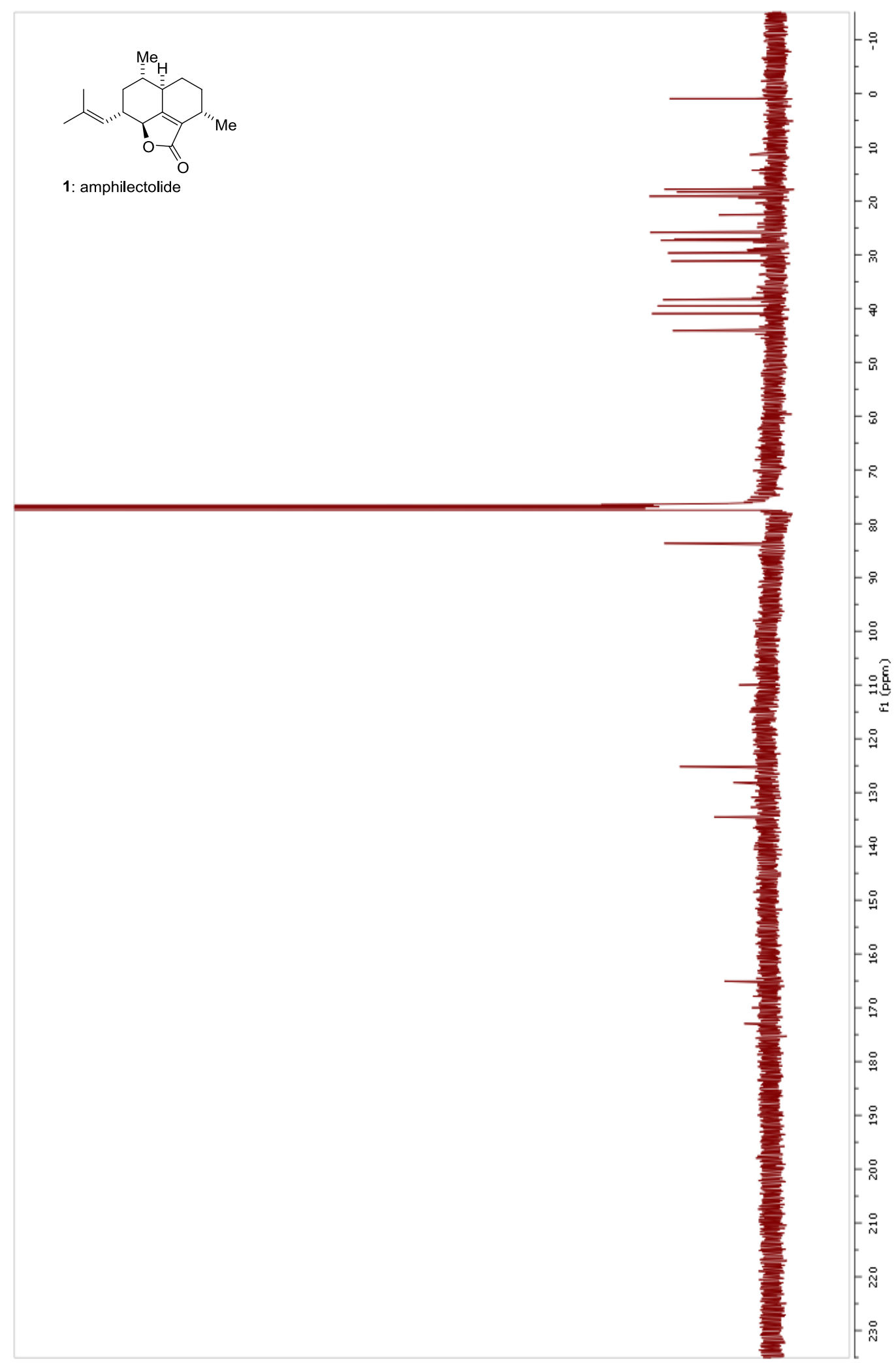




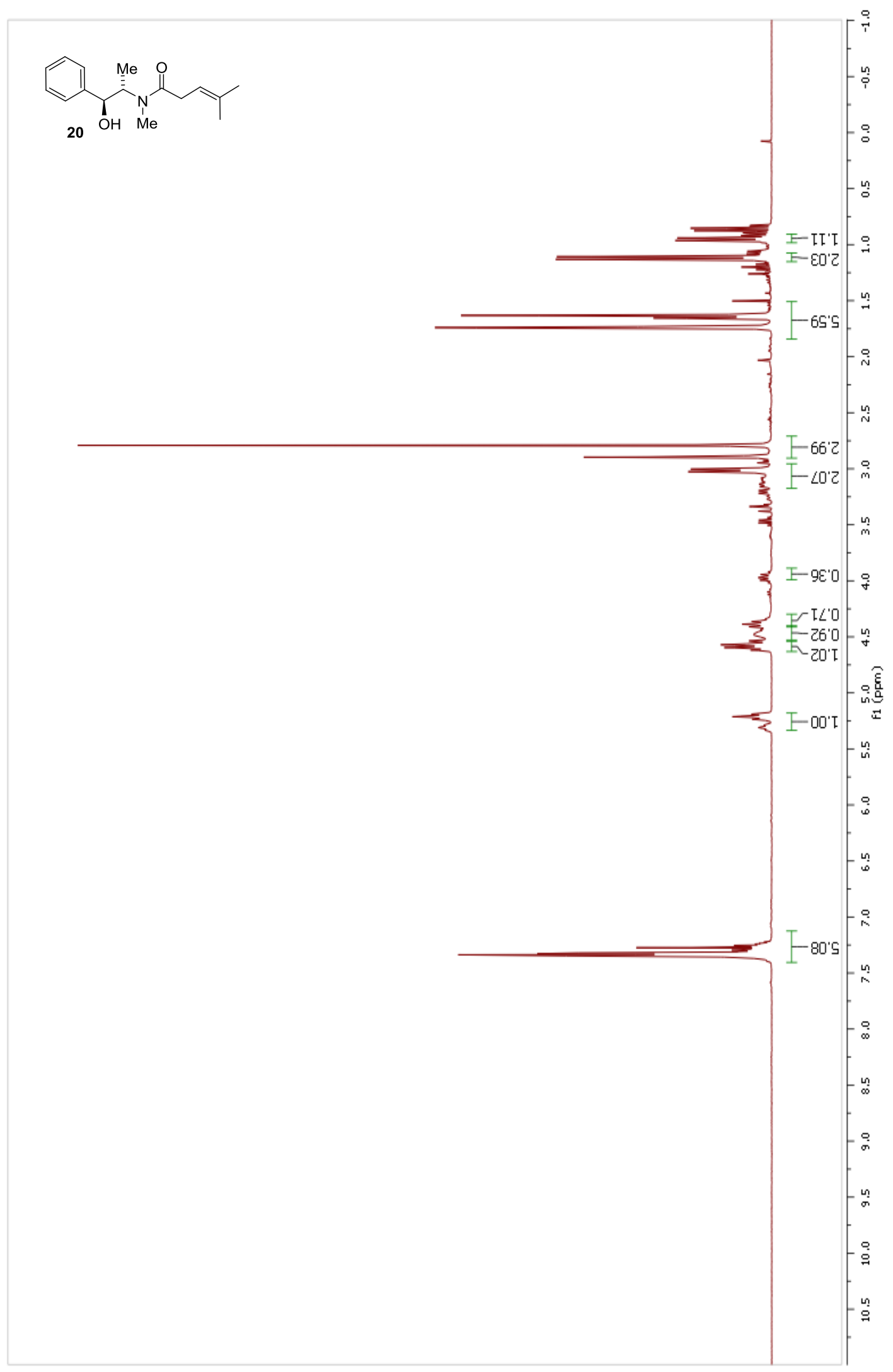


$\overbrace{20}^{\mathrm{OH}}$
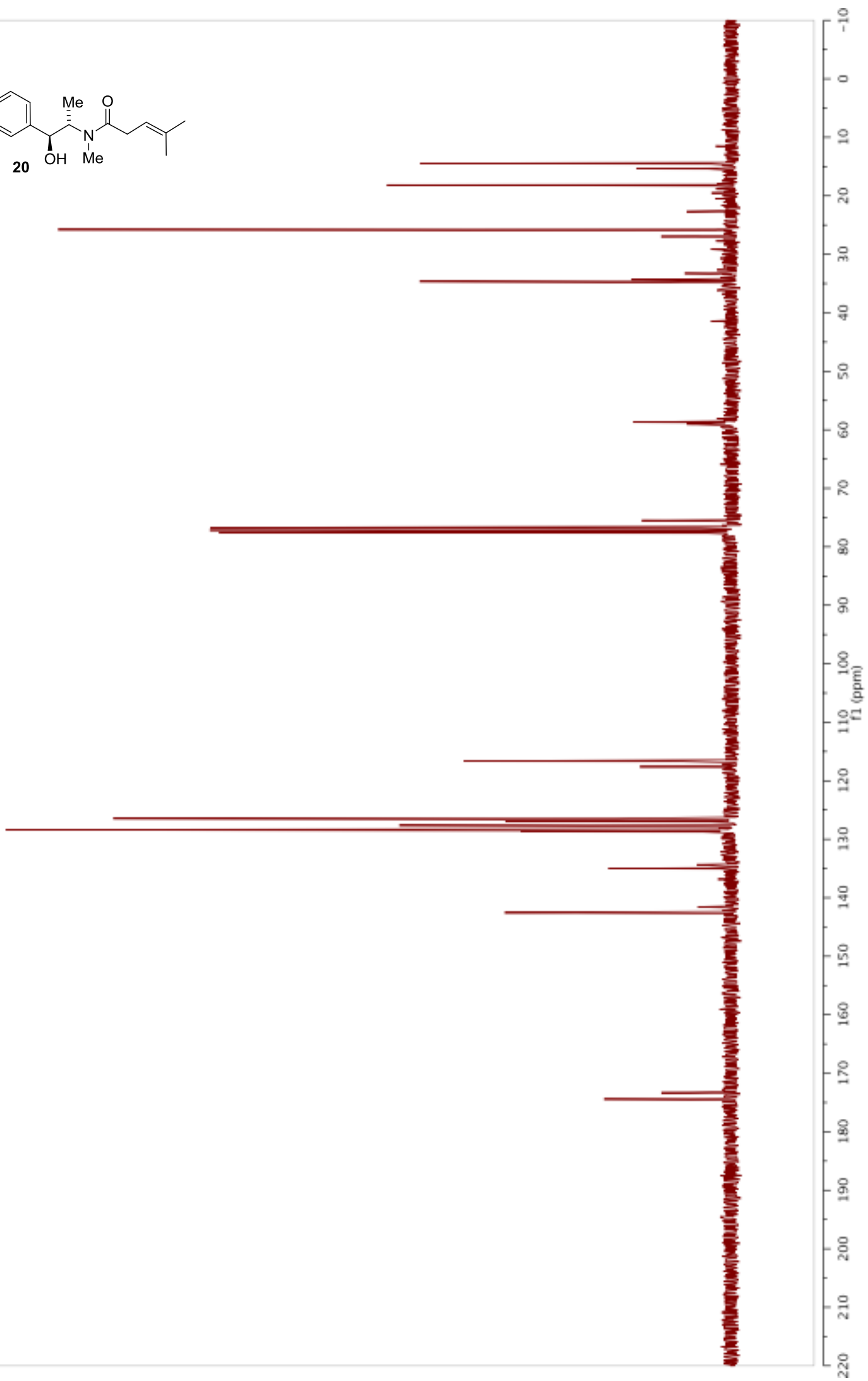


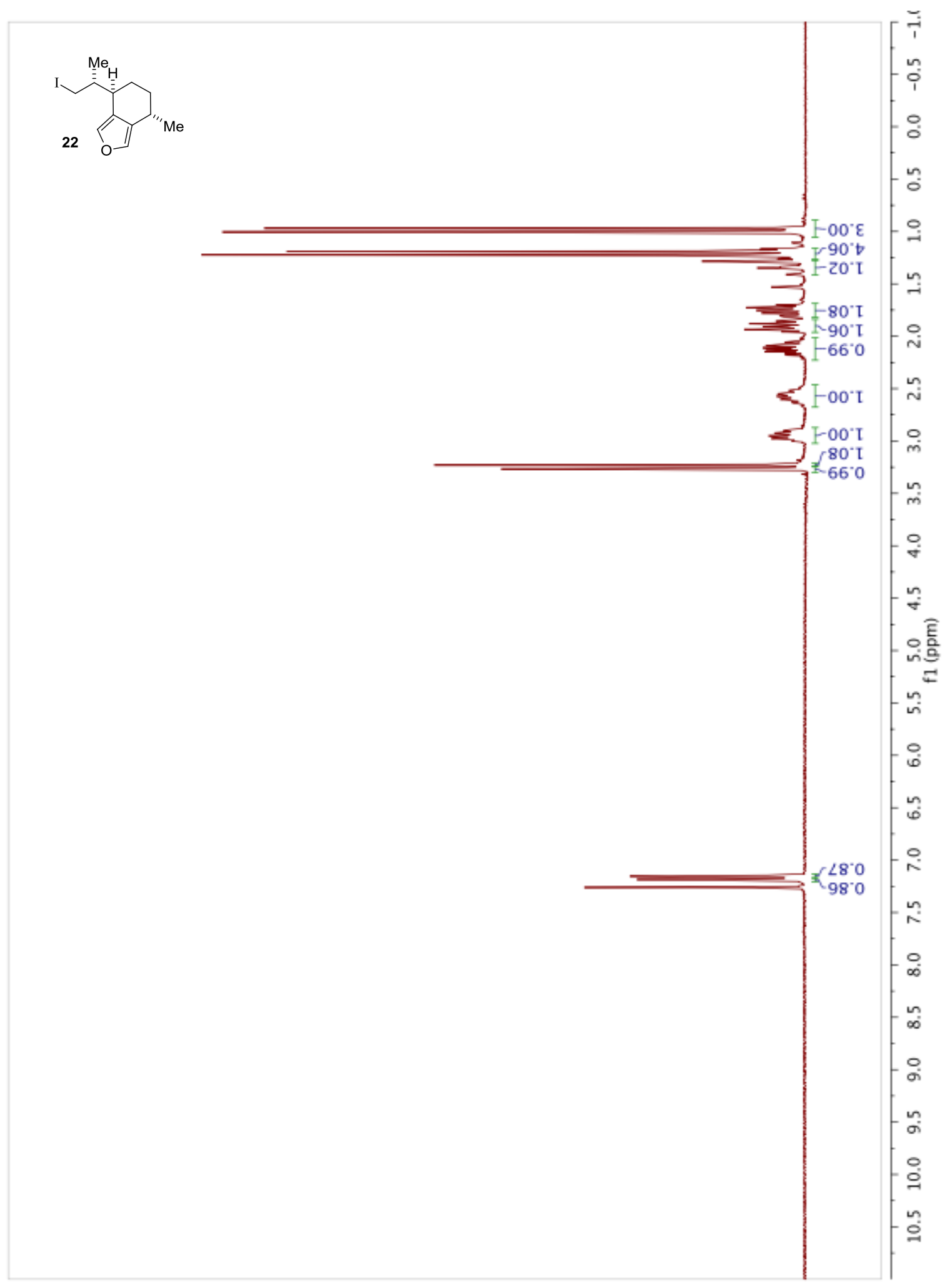




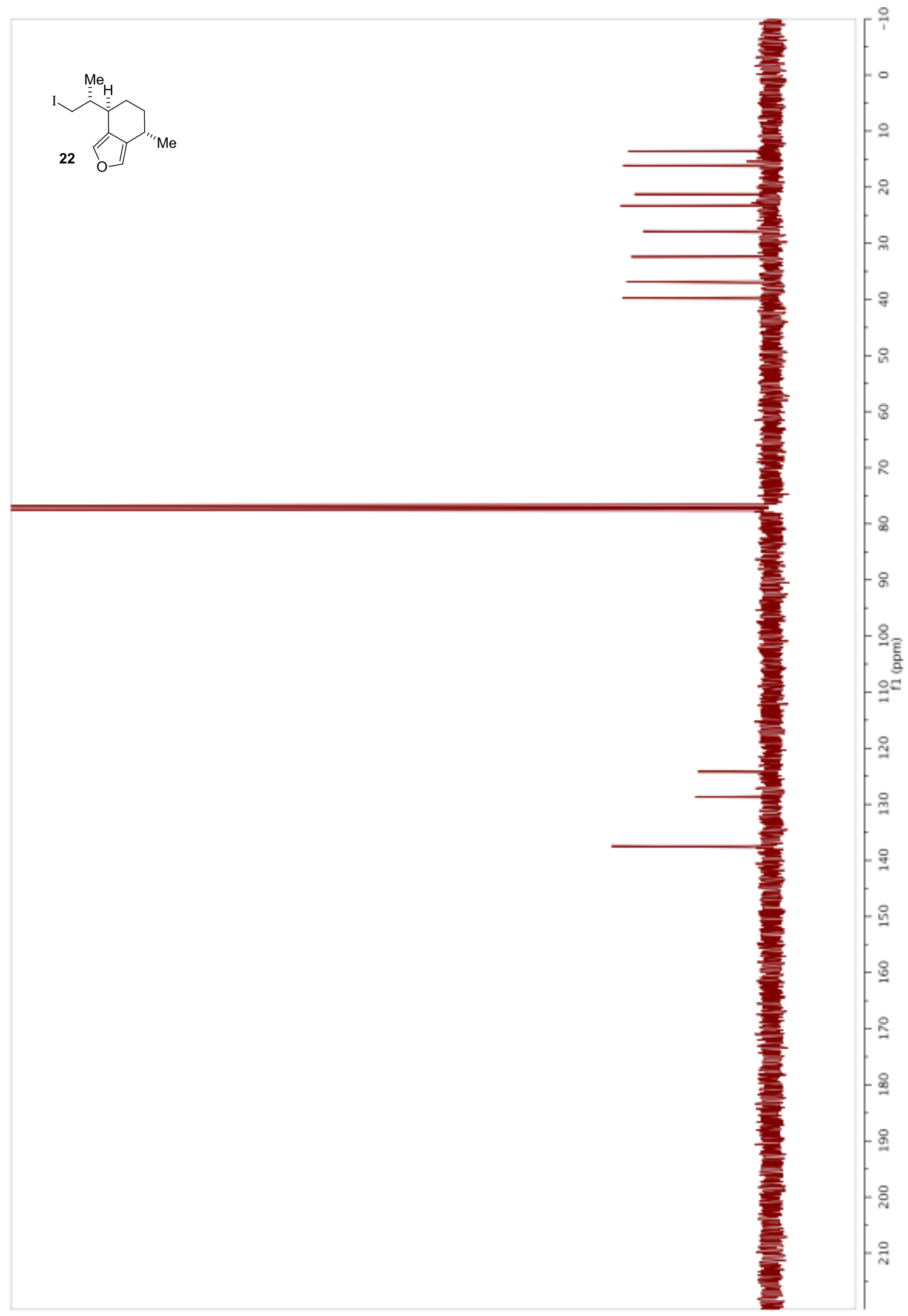

S46 


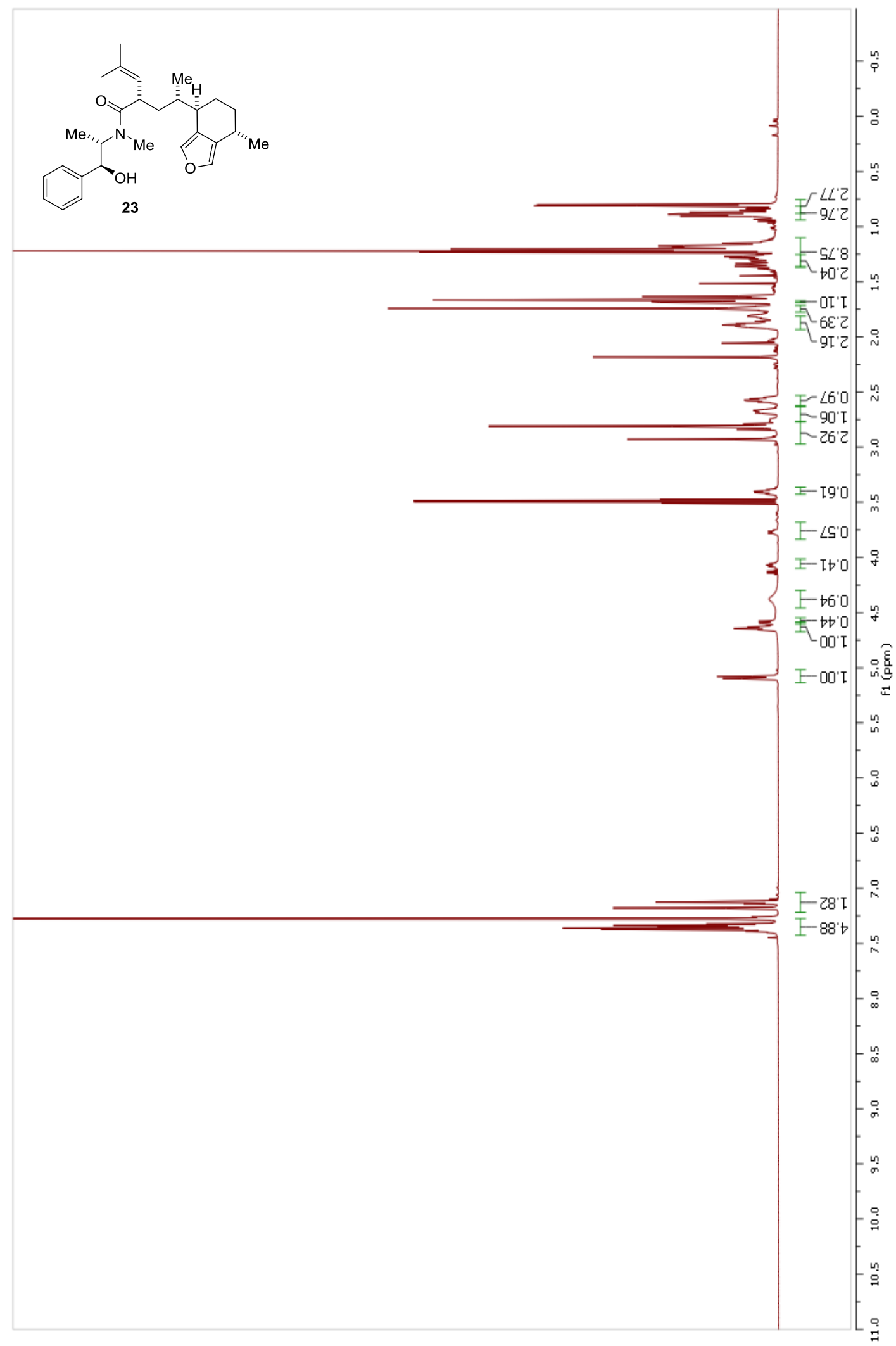




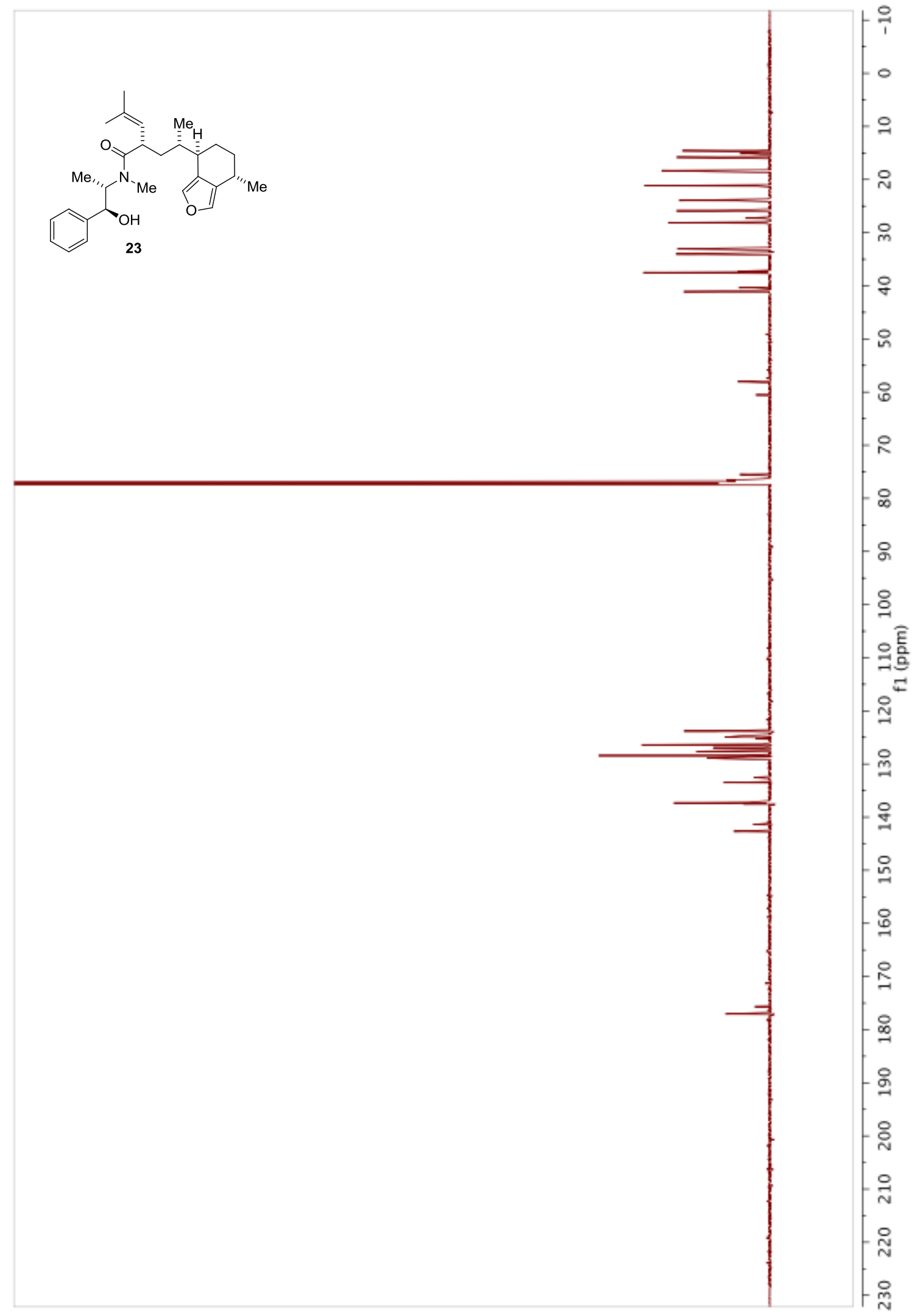




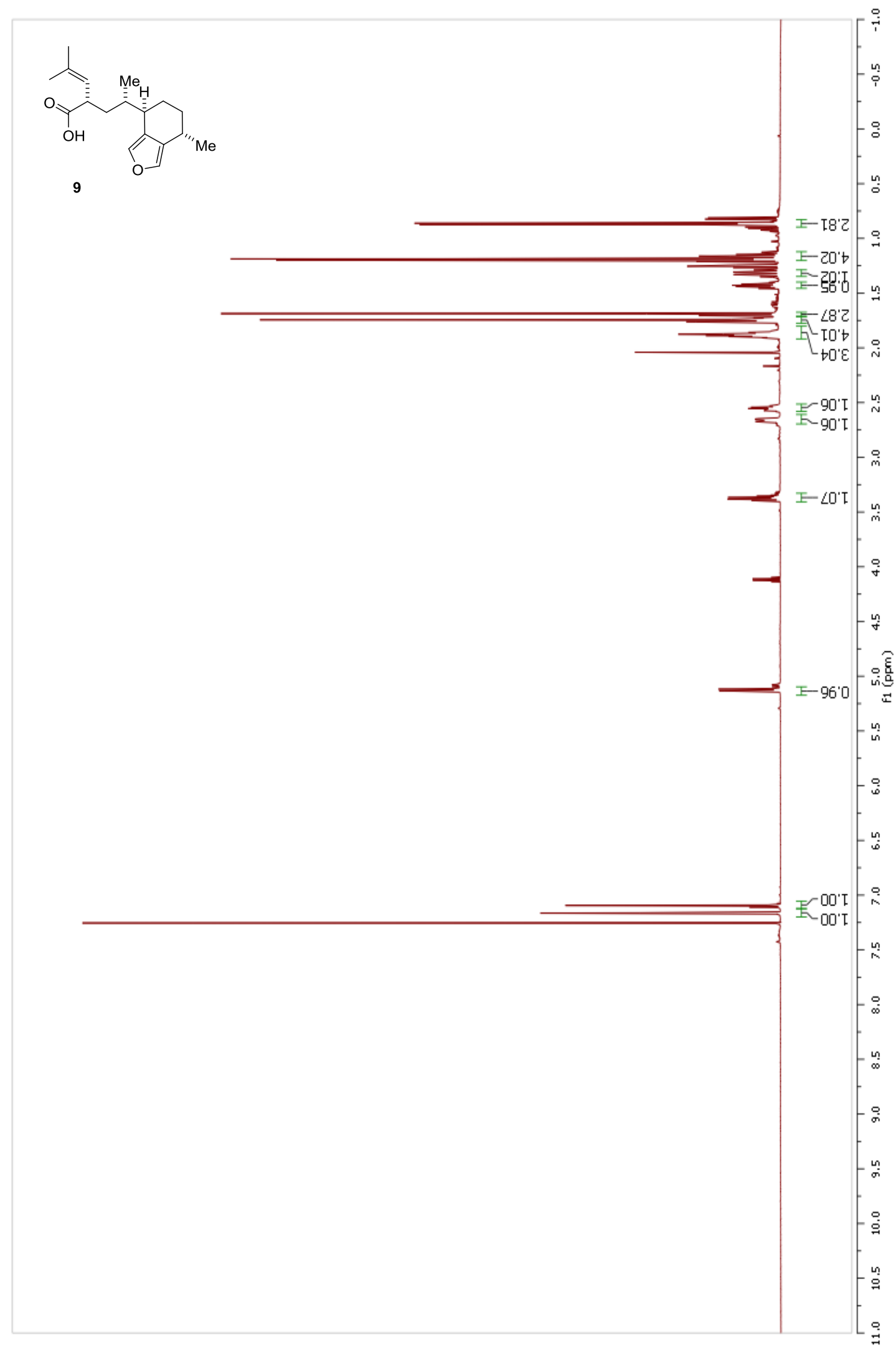



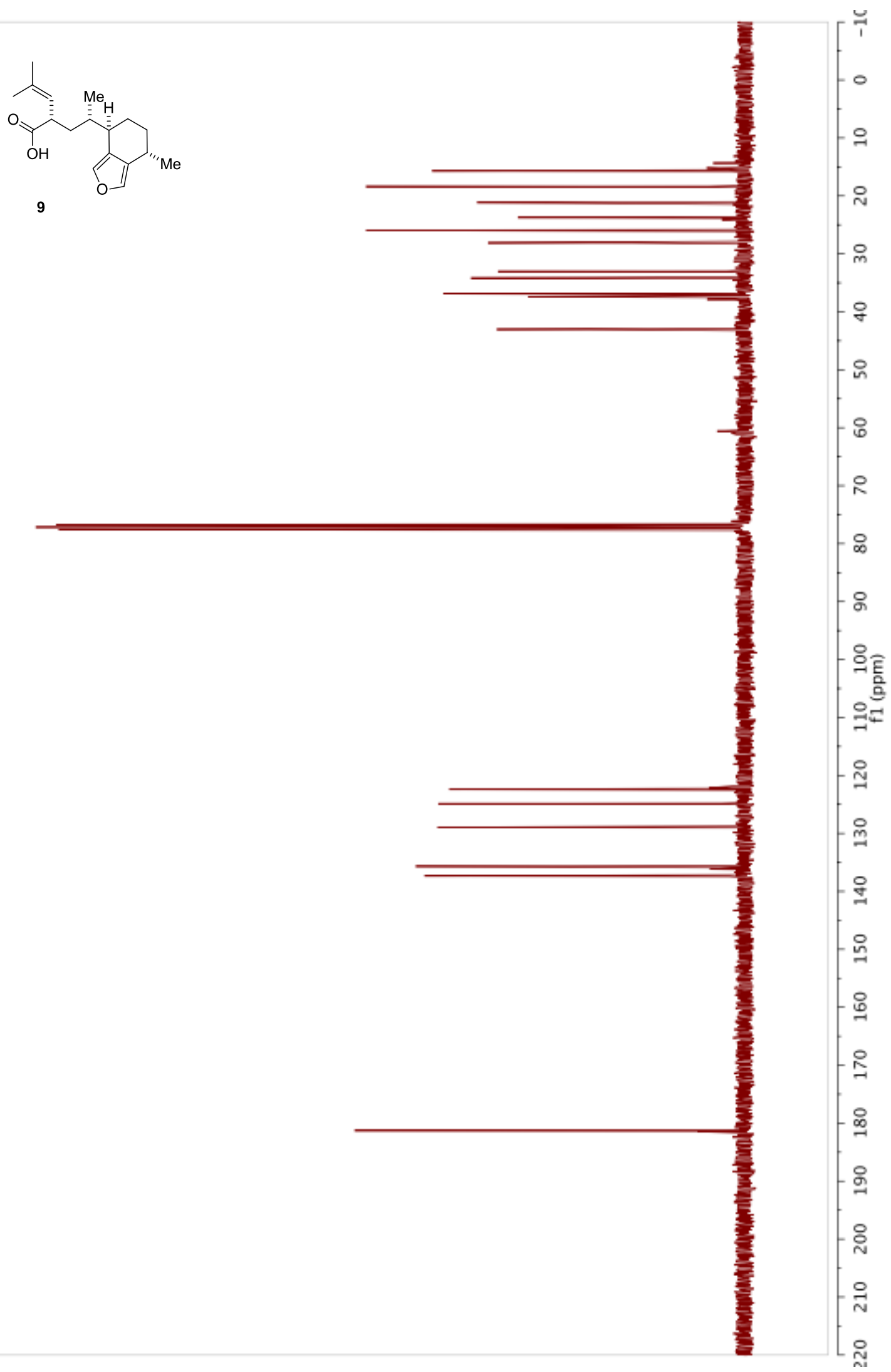


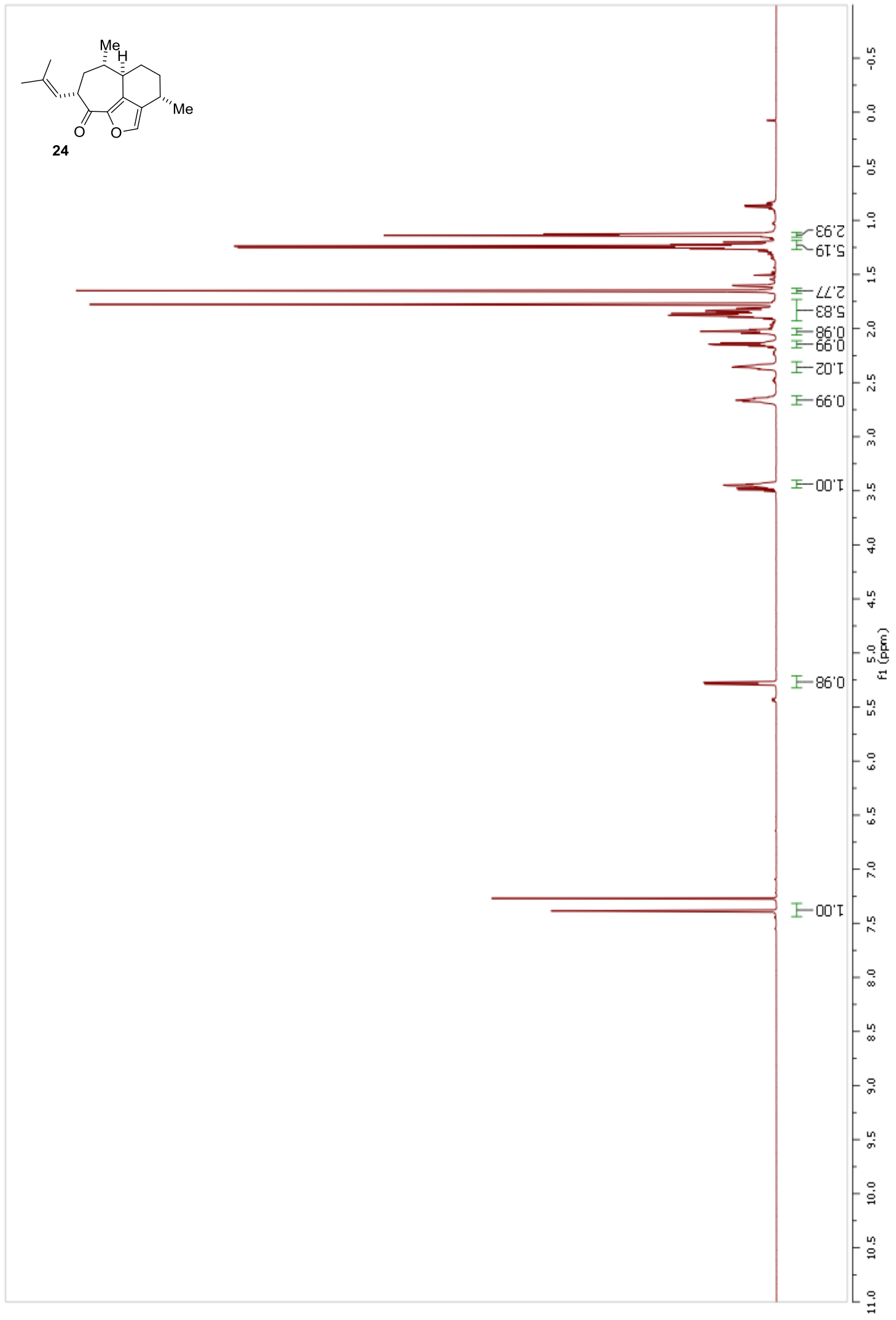



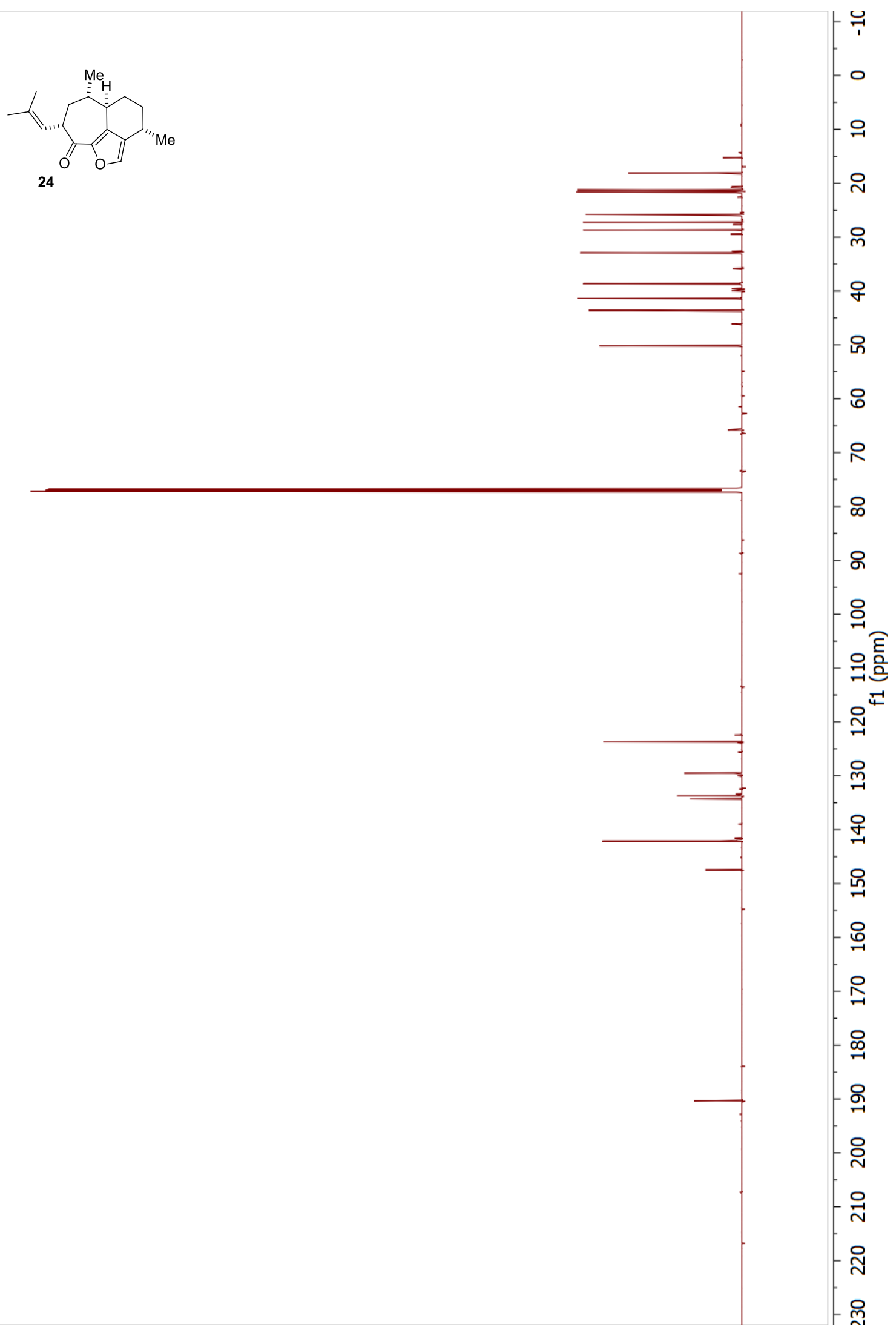

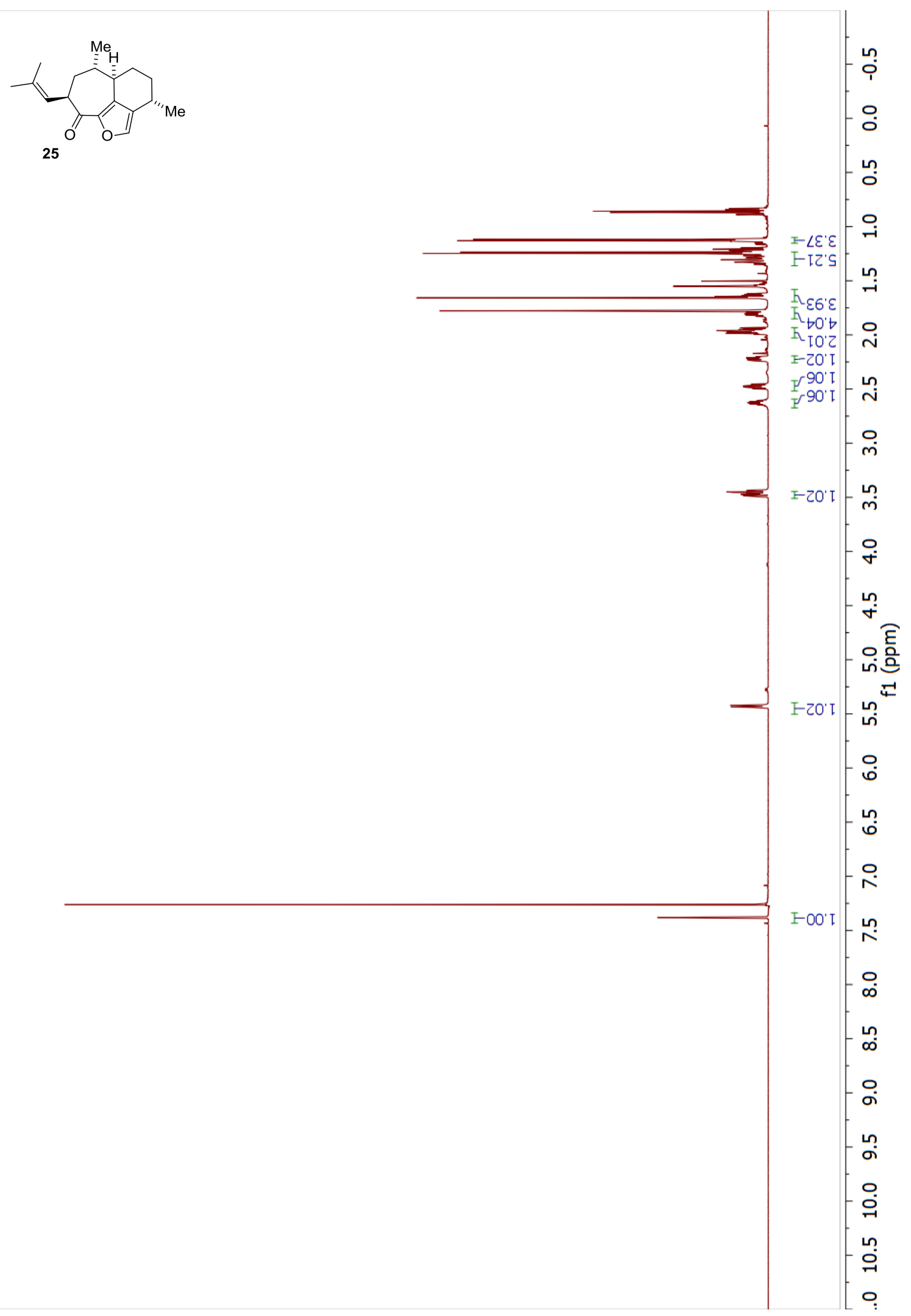


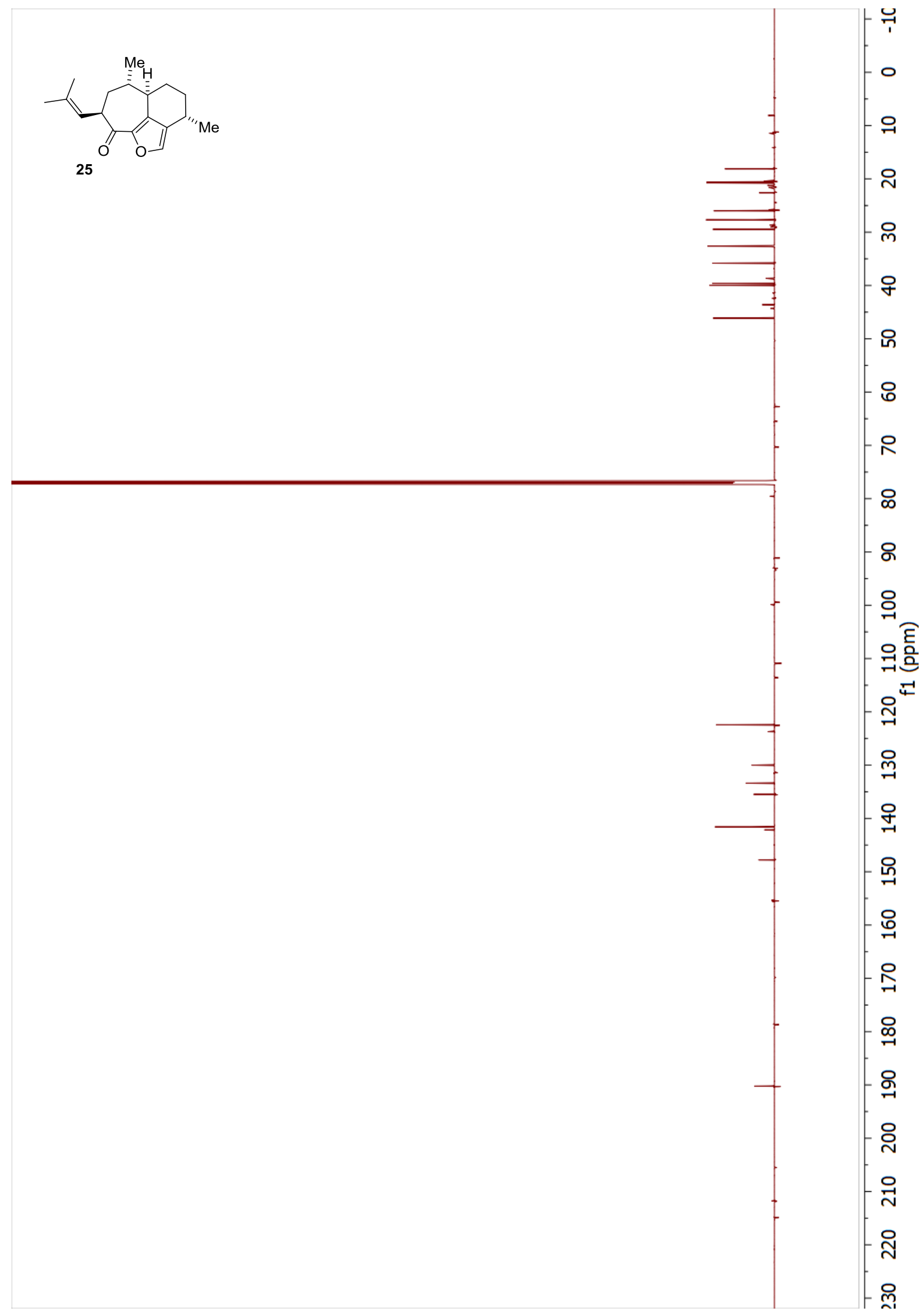




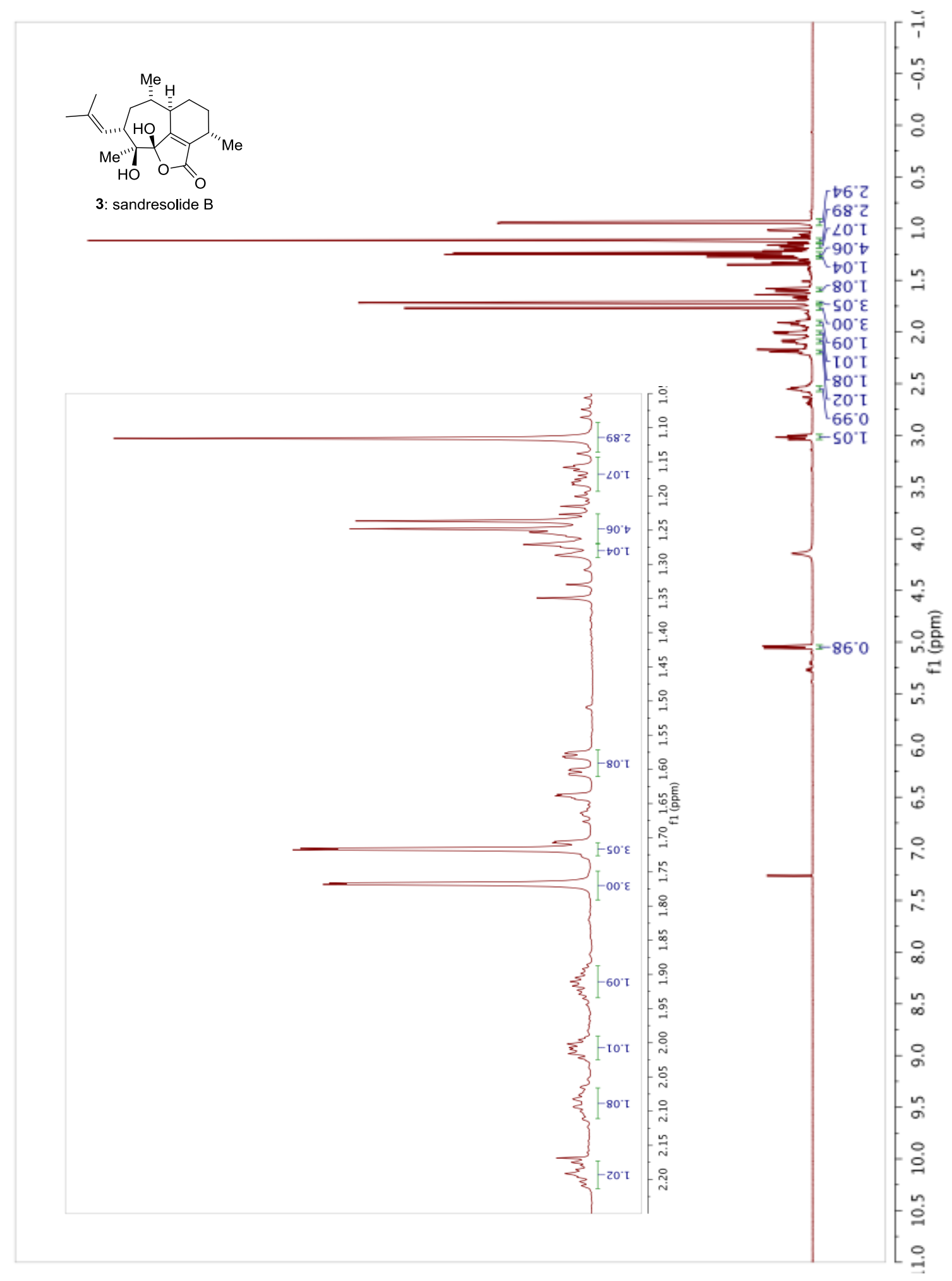




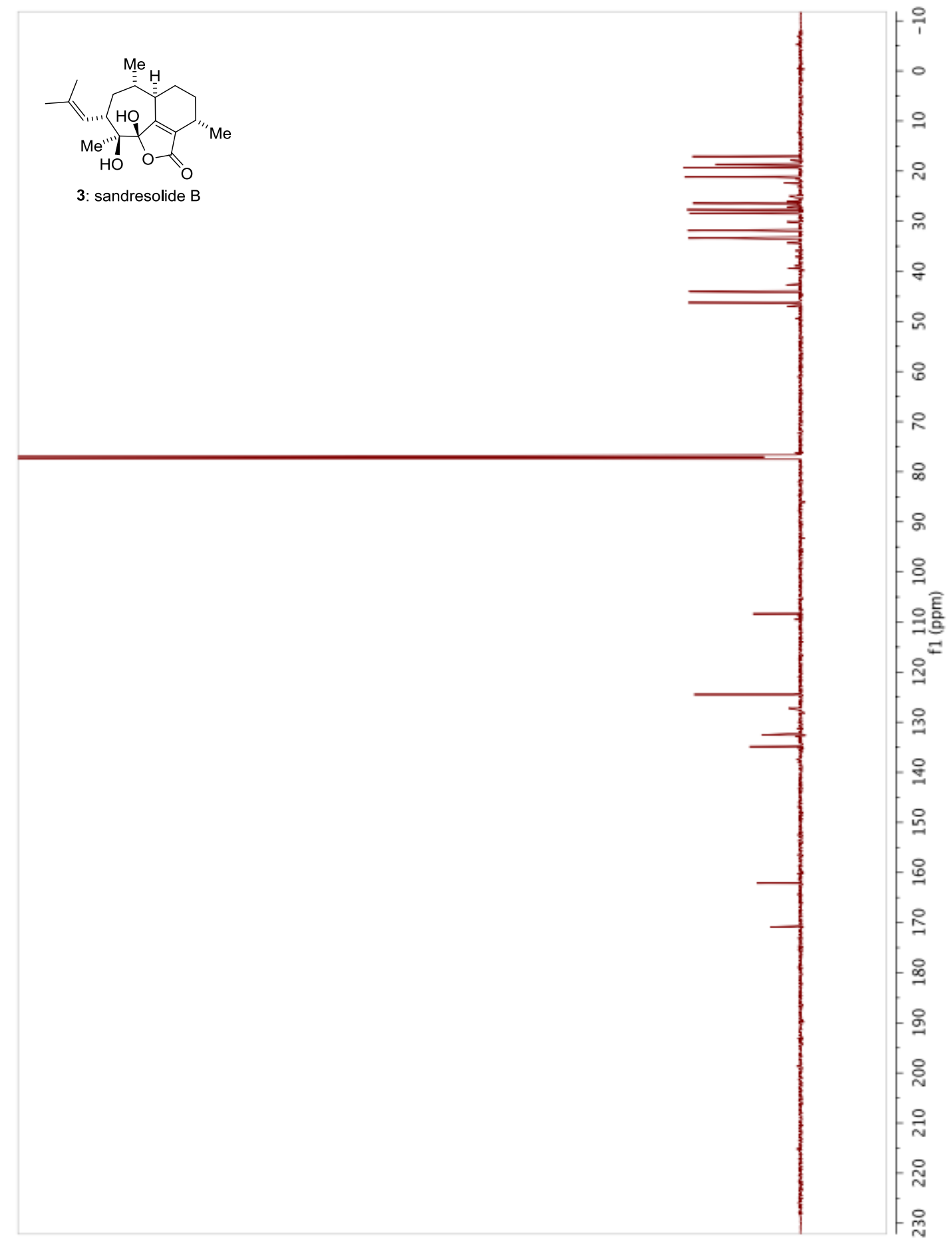


(udd) It

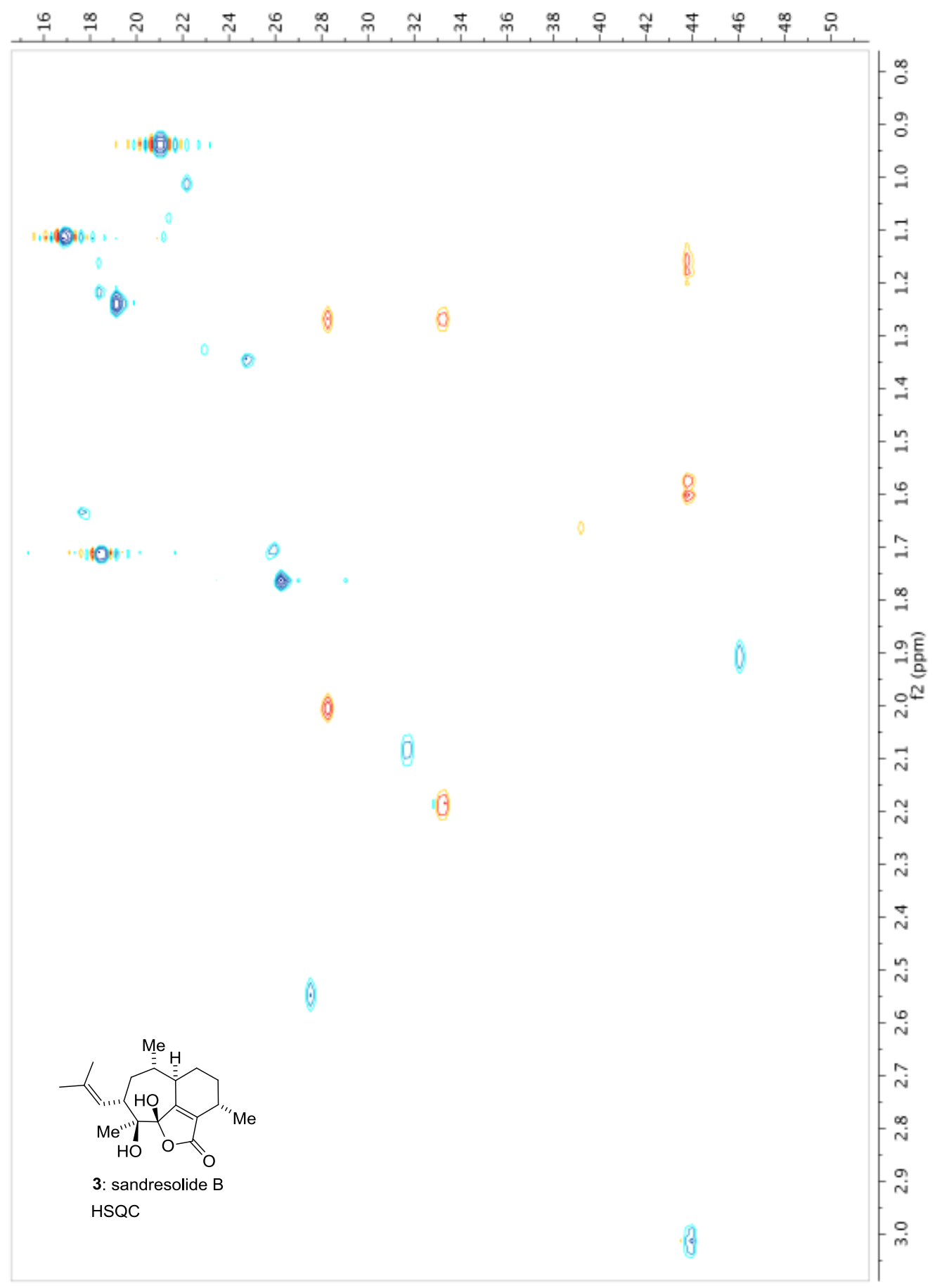




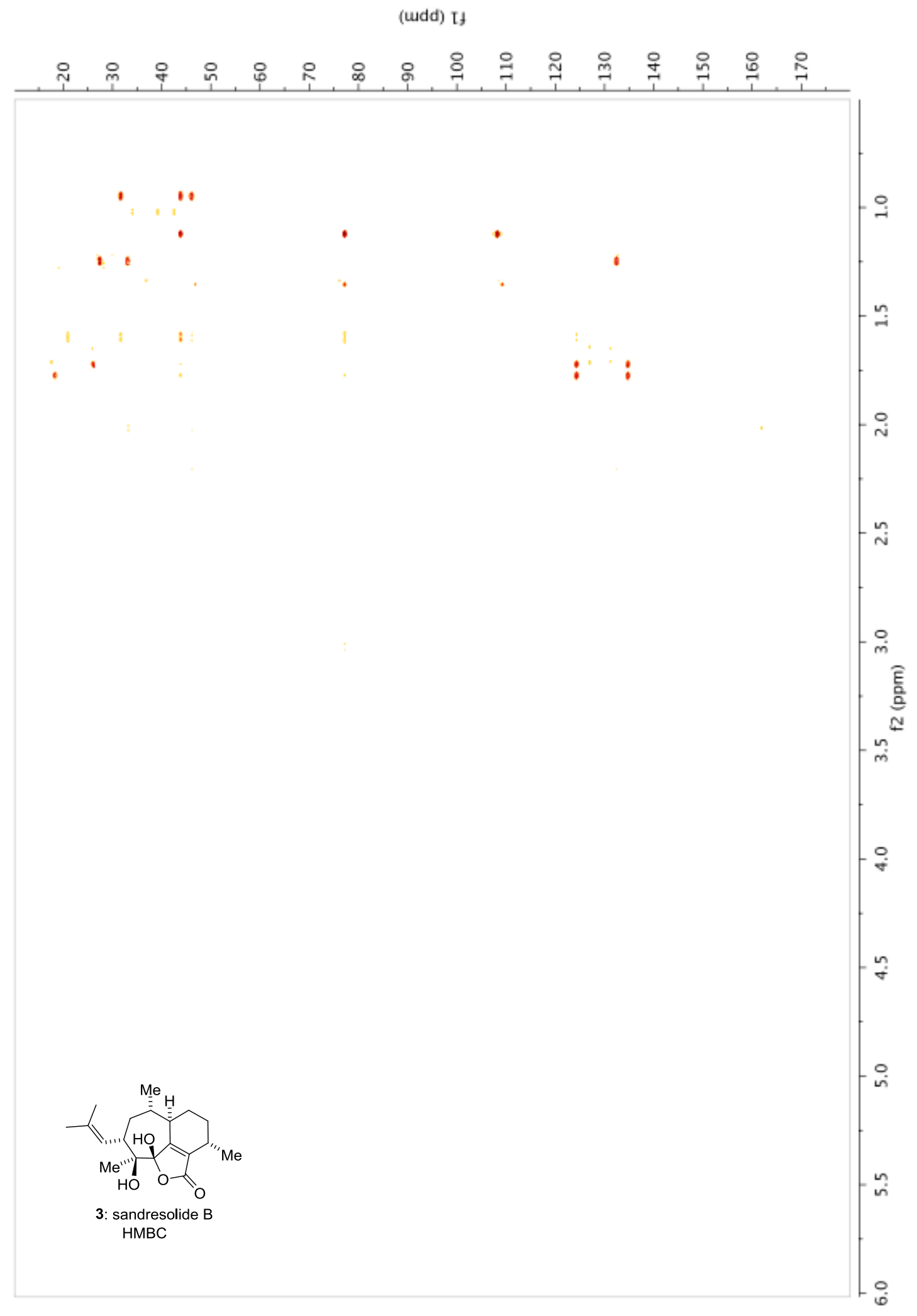




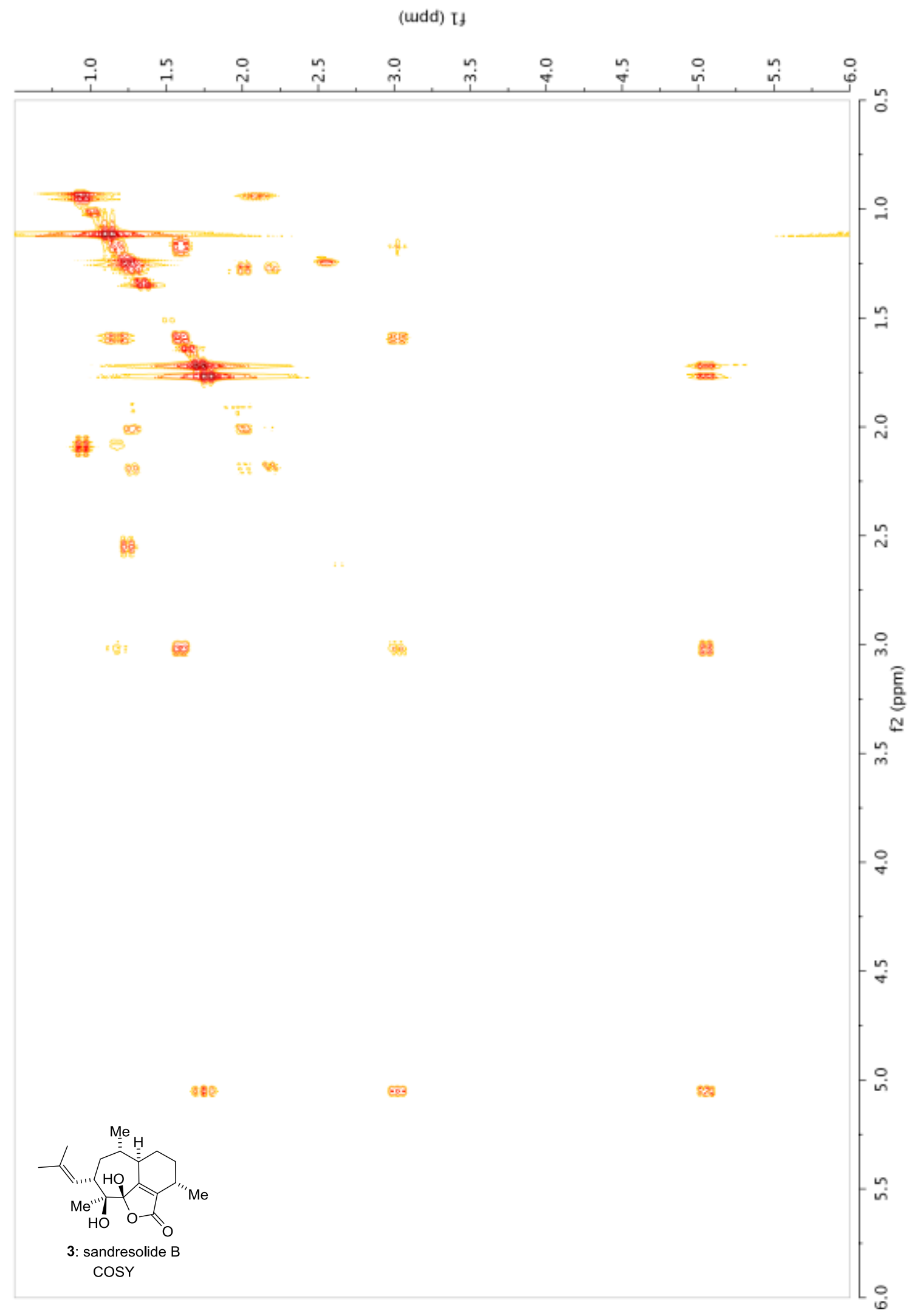

